\title{
Optimality of Debt under Flexible Information Acquisition
}

\author{
Ming Yang * \\ Duke University
}

May 2019

\begin{abstract}
This paper studies a security design problem featuring flexible information acquisition. To raise liquidity, a seller issues a security backed by her asset in place at the price she proposes to a buyer. Before deciding whether to accept the offer, the buyer can acquire costly information about the underlying asset. This case differs from the existing literature on security design, in that the buyer has the full flexibility of choosing not only the amount of resources to spend in information acquisition, but also how to allocate them, depending on the shape of the security. Debt is shown to be the unique optimal security for the seller, as its payoff is the least sensitive to the value of its underlying asset. This minimizes the buyer's incentive to acquire information and mitigates the resulting adverse selection. I do not assume monotonicity of the feasible securities nor impose various distributional assumptions on information structures. Instead, I identify conditions for general information costs that support the results.
\end{abstract}

JEL: D86, D82, G32

Keywords: security design, flexible information acquisition

\footnotetext{
${ }^{*}$ The Fuqua School of Business, Duke University, 100 Fuqua Drive, Durham, NC 27708, ming.yang@duke.edu. An earlier version of this paper is contained in the first chapter of my Princeton graduate thesis. This work is partially supported by the Center for Science of Information (CSoI), an NSF Science and Technology Center, under grant agreement CCF-0939370. I am very grateful to Stephen Morris, Chris Sims, and Hyun Shin for their guidance and support in writing this paper. I also thank Ulf Axelson, Sudipto Bhattacharya, Richard Blundell, Paolo Colla, Martin Cripps, Tri Vi Dang, Jan Eeckhout, Emmanuel Farhi, Diego Garcia, Simon Gervais, Antonio Guarino, Oliver Hart, Barney Hartman-Glaser, Christian Hellwig, Bengt Holmstrom, Arvind Krishnamurthy, Thomas Mariotti, Guillaume Plantin, Adriano Rampini, Morten Ravn, Andrei Shleifer, Alp Simsek, Günter Strobl, Jean Tirole, Dimitri Vayanos, Vish Viswanathan, Michael Woodford, Wei Xiong, Kathy Yuan, Yao Zeng, and seminar participants at Harvard, NYU, Princeton, Stanford GSB, Duke, Toulouse School of Economics, Kellogg, LSE, the 2011 North American Summer Meeting of the Econometric Society, and the 2012 Winter Meeting of the American Economic Association for helpful comments and discussions. All remaining errors are mine.
} 


\section{Introduction}

Asset-backed securities (ABS) are widespread and played a significant role in the 200708 financial crisis. Potential buyers of these securities in the primary markets are mainly informationally sophisticated investors, such as hedge funds, collateralized debt obligation (CDO) managers, and, more broadly, investment banks. These investors actively acquire information about the underlying assets when deciding whether to purchase the ABS. In particular, they have the flexibility of choosing not only the amount of resources to spend in this process but also how to allocate those resources, and their optimal choices depend on the "shape" of the security, that is, the ex ante defined relation between the payoff of the security and the value of its underlying assets.

For example, if the ABS is a debt security, a potential buyer's information acquisition would focus mainly on the downside. That is, whether the total value of its underlying assets will fall short of the face value of the debt security and by how much. This is because the payoff of the security increases one for one with the total value of its underlying assets in that range. The buyer's investigation would not focus on the upside (e.g., whether the total value of the underlying assets will be two or three times of the face value), because the payoff of the security is invariant with the value of the underlying assets in this range. Analogously, if the ABS is instead a call option, whose payoff increases with the total value of its underlying assets only if it is in the money, then opposite to the previous example, the buyer's information acquisition would focus more on the upside than the downside.

Given such flexibility in information acquisition, the potential buyer is able to gain an informational advantage that is endogenous to the design of the security, and can subsequently cherry-pick the security at the expense of the seller. The question then becomes, how can the seller optimally design the security in the first place to mitigate or even deter such adverse selection? This paper addresses this question with a theoretical framework novel in the literature that captures the aforementioned flexibility in information acquisition. The conclusion is that debt is uniquely optimal for this purpose, which is consistent with the prevalence of debt securities in ABS markets.

To fix idea, consider a risk-neutral seller ("she"), ${ }^{1}$ who has an asset in place that generates a random future cash flow. She is impatient and wants to raise liquidity by issuing a security backed by her asset at a price she proposes to a risk-neutral buyer. The buyer ("he") can acquire costly information to evaluate this offer before deciding whether to accept it. The approach that I adopt to model his information acquisition is the key methodological innovation of this paper. While agents in models of canonical contract theory and corporate finance theory are endowed with exogenous signals or restricted to choosing information

\footnotetext{
${ }^{1}$ I will use she/her to refer to the seller and he/his to the buyer of the security throughout the paper. I do not intentionally associate the agents with particular genders.
} 
structures of a particular functional form, ${ }^{2}$ the buyer in my model is allowed to choose any information structure represented by a signal that correlates with the fundamental state in a particular way. ${ }^{3}$ To model this flexibility in information acquisition, I introduce a general cost functional with minimum regularity conditions that assigns non-negative information costs to different information structures. Subject to the cost functional, the flexibility enables the buyer to fine-tune his information acquisition about different fundamental states according to their payoff relevance.

The optimality of debt results from the seller's incentive to discourage the buyer from acquiring information. Such incentive stems from an important feature of the model: fixed aggregate cash flow. That is, the aggregate cash flow to be split between the seller and the buyer is invariant to the success or failure of the transaction, which only reallocates the cash flow. This engenders conflict of interest between the two parties: the buyer gains from information acquisition at the expense of the seller through adverse selection. That is, he takes advantage of the flexibility of information acquisition to differentiate states with different security payoffs, and subsequently rejects (accepts) the offer at states unfavorable (favorable) to him but possibly favorable (unfavorable) to the seller. Anticipating this, the seller wants to design a security with a constant payoff to minimize the buyer's incentive to acquire information. This gives rise to the flat tail of the debt security. When the underlying cash flow becomes too low to support such constant payoff, the limited liability constraint binds and yields the 45-degree-line portion of the debt security.

I also provide a number of robustness results to explore which model assumptions drive which aspect of the optimal security. Specifically, I consider two alternative settings. One involves alternative assumptions about the information cost functional. The other considers the possibility of issuing a security backed by multiple underlying assets. Fixed aggregate cash flow remains the key feature in these settings. The intuition of the baseline model carries over, and debt securities remain optimal.

Although ubiquitous, debt is not always the optimal way to obtain external financing in practice. Consider the following variation of the model. Instead of being generated from some asset in place, the random cash flow comes from a project that can be undertaken only if the security backed by it is accepted by the buyer in exchange for the upfront investment required. In this case, aggregate cash flow depends on whether the transaction succeeds, and hence, information acquisition is valuable. The resulting optimal security encourages information acquisition, and debt is no longer optimal. Another situation in which debt may not be optimal is that where multiple buyers are acquiring information and bidding for the security. In that case, endogenous asymmetric information between the buyers

\footnotetext{
${ }^{2}$ One prominent example is Gaussian signals, or more generally, signals with additive noise. Information acquisition is then modeled as reducing the magnitude of noise at some cost.

${ }^{3}$ In the security design problem, the fundamental state is the underlying cash flow. I use these two terms interchangeably throughout the paper.
} 
emerges, resulting in the winner's curse and reducing the seller's revenue. Since the degree of information asymmetry could be non-monotonic in information acquisition, it is unclear whether the seller wants a flat security to discourage information acquisition.

The contribution of this paper is twofold. First, since my model allows arbitrary information choices under a very general class of information costs, the optimality of debt in the case of information deterrence established here greatly enhances the robustness of similar results in existing papers on security design that feature information acquisition and adverse selection. Second, in terms of the methodological contribution, this paper develops a very general modeling technique to capture the incentives of relevant parties in the presence of flexible information acquisition, and demonstrates how this technique can be incorporated into canonical contract theory and corporate finance theory models, opening a new arena for the study of strategic interactions in agency problems.

I proceed as follows. Section 2 presents the baseline model and analyzes the special case of zero information cost. Section 3 introduces flexible information acquisition in a decision problem, and Subsection 3.1 lays out the foundation for analyzing the players' behavior in the subsequent trading game. Section 4 derives the unique optimal security in various circumstances and discusses the underlying mechanism. Section 5 considers several alternative settings and variations of the baseline model, showing the robustness and limitations of the results. Section 6 concludes and discusses some related issues. Appendix A discusses the assumptions of flexible information acquisition and their implications. All proofs are in Appendix B.

Related Literature. By considering a more general class of information costs, my approach of flexible information acquisition extends the rational inattention approach pioneered by Sims (1998). My paper also differs from the rational inattention literature in that I focus on agents' flexibility in acquiring information instead of their limited capacity in processing information.

My paper is closely related to the security design literature. I focus this review on the most closely related papers, based on a taxonomy of models that differ in the source of information asymmetry and the value of private information (acquisition).

In the first class of models, the seller is endowed with private information. Information is detrimental, as it creates a downward-sloping demand curve that forces high-quality sellers to sell less. To mitigate this problem, the seller designs information-insensitive securities. In DeMarzo and Duffie (1999), an informed seller sells the security in a Leland and Pyle (1977) type of signaling game. Debt is optimal within the class of monotone securities when the seller's information structure admits a uniformly worst case. My model differs from that of DeMarzo and Duffie (1999) in the following aspects. In my setting, bargaining power and information advantage are separated between the two parties. Illiquidity is not a result of signaling with exogenous information asymmetry but stems from adverse selection caused by 
information acquisition. Debt is optimal because it discourages information acquisition in the first place, rather than being insensitive to existing private information. Thus, I identify conditions on the production, as opposed to the structure, of information such that debt is uniquely optimal, and not just among the monotone securities. DeMarzo (2005) argues that pooling destroys the seller's ability to signal the quality of the assets separately but reduces the information sensitivity of the senior tranche. When the second effect dominates, pooling and tranching are optimal. Subsection 5.3 shows that pooling and tranching are always optimal, since my setting does not involve the signaling effect but has a similar second effect: pooling and tranching make each individual asset less payoff relevant and reduce the incentive to acquire information. Biais and Mariotti (2005) and Nachman and Noe (1994) also belong to this class of models. The former extends the analysis of DeMarzo and Duffie (1999) to accommodate buyers' market power, while the latter considers security design after the arrival of private information. They identify conditions under which debt is optimal among monotone securities. ${ }^{4}$

Unlike the literature on security design featuring signaling, Axelson (2007) highlights private information on the buyer side. An uninformed seller sells a security to exogenously informed buyers in a uniform price K-unit auction. The security is underpriced when its Kth and $\mathrm{K}+1$ 'st highest conditional expectations differ from each other. Hence, the underpricing stems from the heterogeneity of these two buyers' valuations, which is strong for intermediate values and weak for low and high values of the fundamental, provided that the monotone likelihood ratio property holds. Within the class of dual-monotone securities, it is optimal to make the security slope equal to 1 when underpricing is weak and 0 when it is strong, resulting in a combination of debt and a call option. This mechanism differs from those in my model and in the aforementioned signaling literature. Information is not detrimental. Instead, it helps adjust the scale of investment according to the particular opportunity. It is the imperfection of information that creates heterogeneous valuations and the underpricing problem. ${ }^{5}$ Inderst and Mueller (2006) also consider security design with the buyer's private information and find that either debt or a call option is optimal. Their model features exogenous information and variable aggregate cash flows and thus differs from mine.

The third class of models studies security design in the presence of information acquisition. In a setting similar to my baseline model, Dang, Gorton, and Holmström (2015) model information acquisition via the costly state verification (CSV) approach inspired by Townsend (1979) and show that there are an infinite number of optimal securities, called quasi-debt. Like a standard debt contract, the payoff of a quasi-debt security equals the value of the underlying asset when it falls below the price of the security. But the pay-

\footnotetext{
${ }^{4}$ Faure-Grimaud (2000) also belongs to this class of models. In a different context, debt is shown to be optimal and renegotiation-proof.

${ }^{5}$ In addition, the auction setting allows for a formal analysis of competition, showing that more intensive competition leads to more equity-like securities, a result not present in my model nor in most signaling models of security design.
} 
off of such a security can take arbitrary values above the price whenever feasible. This is because, as CSV perfectly reveals the state, the buyer's optimal strategy is to accept the offer regardless of the "shape" of the security as long as it pays off more than its price. In contrast, in my framework with flexible information acquisition, standard debt is uniquely optimal. This is because, it is now optimal for the buyer to acquire information that better differentiates states with greater differences in the security payoff that justify the cost of doing so. To discourage such practice and mitigate the resulting adverse selection, the seller should make the security payoff as flat as possible even if the security pays off more than its price, resulting in the flat tail of standard debt. Moreover, this paper and Dang, Gorton, and Holmström (2015) also differ in their focuses. The latter focuses on the macroeconomic implications of debt, while the former examines the microfoundation of debt via the approach of flexible information acquisition. In Boot and Thakor (1993) and Fulghieri and Lukin (2001), a firm (seller) is endowed with private information about its project. Good firms want to separate themselves from bad ones by issuing information-sensitive securities to encourage buyers' information acquisition, which makes the equilibrium price more informative. Debt becomes optimal when information is expensive. In contrast to Dang, Gorton, and Holmström (2015) and this paper, the two parties start with asymmetric information, and information acquisition helps reduce information asymmetry, making information acquisition valuable and non-debt securities optimal. In a simple bargaining setting, Farhi and Tirole (2015) show that tranching (i.e., issuing risk-free debt), instead of selling the whole asset, encourages (discourages) information acquisition when it is socially desirable to discourage (encourage) it under certain conditions. In a noisy rational expectation framework, Hennessy (2013) shows a similar effect through the channel of interaction between tranching and information aggregation in competitive markets. The analysis in these two papers is more general than mine, in the sense that they examine various situations: both parties start with symmetric ignorance, or only one party is informed. It is also more restrictive because of its binary-state setting and focus on tranching, rather than on general security design.

Finally, the concept of flexibility in security design as presented in this paper has recently gained momentum. Instead of focusing on adverse selection caused by flexible information acquisition, Hébert (2017) studies a flexible moral hazard problem based on Holmstrom and Milgrom (1987), in which the agent can choose any distribution of cash flows, each associated with a cost. Debt is optimal when the cost is measured by Kullback-Leibler divergence. Debt may not be optimal under more general forms of divergence. Although Hébert (2017) and my model share the same concept of flexibility, they differ in nature, and the modeling approaches are not directly comparable. Another recent paper in this area is Antic (2015). In addition to the agent's flexibility in choosing the distribution of cash flows, the principal is ambiguity-averse. The optimal security approaches debt when the ambiguity 
aversion vanishes and approaches equity in the presence of large ambiguity aversion.

\section{The Model}

I present my baseline model of liquidity provision. It does not aim to capture every aspect of liquidity provision, such as risk sharing, market competition, or reselling, but highlights the flexibility of information acquisition and its effects on optimal security design.

I consider a two-date game with two players. A seller is endowed with an asset at date 0 that generates a verifiable random cash flow $\theta \in \Theta=\mathbb{R}_{+}$at date 1 , while a potential buyer owns consumption goods at date 0 . Following the convention of the security design literature, I assume that both players are risk neutral. Specifically, player $i$ 's utility function is given by

$$
u_{i}=c_{i 0}+\delta_{i} \cdot c_{i 1}
$$

where $c_{i t}$ denotes player $i$ 's consumption at date $t$ and $\delta_{i} \in[0,1]$ is the player's subjective discount factor, and $i \in\{s, b\}(\{s, b\}$ stands for $\{$ seller, buyer $\})$. I assume $\delta_{b}>\delta_{s}$, that is, the seller is less patient than the buyer. An alternative interpretation is that the seller has a better investment opportunity or higher cost of carry than the buyer. This assumption creates gains from trade. Both parties may benefit from transferring some goods to the seller at date 0 and compensating the buyer with security payoff backed by the random cash flow $\theta$ at date 1 .

To focus on the adverse selection resulting from information acquisition, I assume that both players start with identical information about $\theta$, represented by a full support common prior $P$ over $\mathbb{R}_{+} . \quad P$ is assumed to be absolutely continuous with respect to Lebesgue's measure on $\mathbb{R}_{+}$. I also assume that the expectation $\mathbb{E} \theta$ exists.

A security backed by the asset is a mapping $s: \mathbb{R}_{+} \rightarrow \mathbb{R}_{+}$such that $\forall \theta \in \mathbb{R}_{+}, s(\theta) \in$ $[0, \theta]$. That is, I require the security to respect both players' limited liability constraints. An offer $(s(\cdot), q)$ is a security $s(\cdot)$ associated with a price $q>0$. Throughout the baseline model, I assume that it is the seller who makes an offer, and the buyer acquires information about $\theta$ before deciding whether to accept the offer. I denote the buyer's decision by $a \in\{0,1\}$, with $a=1$ referring to "accept" and $a=0$ to "reject," respectively. ${ }^{6}$

The main result of this paper is that the optimal security is always a debt security in the sense that it possesses the two defining features of debt: i) a 45-degree-line portion and ii) a flat tail. In particular, the optimal security $s^{*}$ takes the form $s^{*}(\theta)=\min \left(\theta, D^{*}\right)$, where $D^{*}$ is a positive face value. It has a flat tail in the sense that the security payoff is constant once the future cash flow $\theta$ exceeds face value $D^{*}$. When the cash flow falls short of the face

\footnotetext{
${ }^{6}$ To focus on security design under flexible information acquisition, the main innovation of this paper, I simplify the bargaining process by assuming that the seller makes a take-it-or-leave-it offer. I consider a reduced-form allocation of bargaining power in the online appendix.
} 
value, all the cash flow goes to the buyer, resulting in the 45-degree-line portion of a debt security.

\subsection{The Special Case of Zero Information Cost}

Before exploring the full-fledged model with costly information acquisition, I first develop some insight by examining the special case of zero information cost. This amounts to letting the buyer directly observe the future cash flow $\theta$. The buyer's optimal decision in this case is straightforward. He accepts any offer $(s(\cdot), q)$, if and only if his discounted security payoff $\delta_{b} s(\theta)$ exceeds the price $q$. It immediately follows that the seller will never set $s(\theta)>\delta_{b}^{-1} q$ for any $\theta$. Otherwise, she can be strictly better off by slightly reducing the security payoff $s(\theta)$ without affecting the chance of acceptance of the offer. The seller thus sets either $s(\theta)<\delta_{b}^{-1} q$ or $s(\theta)=\delta_{b}^{-1} q$. When $s(\theta)<\delta_{b}^{-1} q$, the offer is rejected, and the seller's payoff is zero. When $s(\theta)=\delta_{b}^{-1} q$, the offer is accepted, and the seller's payoff, given $\theta$, is ${ }^{7}$

$$
q-\delta_{s} s(\theta)=q\left(1-\delta_{s} \delta_{b}^{-1}\right)
$$

Hence the seller's expected payoff is

$$
\operatorname{Pr}\left(s(\theta)=\delta_{b}^{-1} q\right) \cdot q\left(1-\delta_{s} \delta_{b}^{-1}\right)
$$

For any price $q$, this expected payoff is maximized when the probability of acceptance $\operatorname{Pr}\left(s(\theta)=\delta_{b}^{-1} q\right)$ is maximized. Thus, for all $\theta \geq \delta_{b}^{-1} q$ the optimal security payoff is $\delta_{b}^{-1} q$.

It is straightforward to see that any security $s_{q}$ that satisfies $s_{q}(\theta)=\delta_{b}^{-1} q$ for $\theta \geq \delta_{b}^{-1} q$ is optimal and that the debt security $\min \left(\theta, \delta_{b}^{-1} q\right)$ is one such security. But there also exist infinitely many other optimal securities. This is because, for any $\theta<\delta_{b}^{-1} q, s_{q}(\theta)$ can take arbitrary values in $[0, \theta]$ and thus does not have to feature a 45 -degree-line portion. This lack of the first defining feature of debt stems from the buyer's ability to distinguish between different states of $\theta$ at no cost. In particular, the buyer can perfectly identify those $\theta$ below $\delta_{b}^{-1} q$ and reject the offer. Hence, the value of $s_{q}(\theta)$ does not matter to the seller's payoff, and non-uniqueness is not essential in this special case. In contrast, all the optimal securities feature a flat tail: $s_{q}(\theta)=\delta_{b}^{-1} q$ for $\theta \geq \delta_{b}^{-1} q$. This is because, given that the buyer can perfectly observe $\theta$ and thus know $s(\theta)$, choosing $s(\theta)<\delta_{b}^{-1} q$ causes rejection and forgoes the seller's gain from the trade, while choosing $s(\theta)>\delta_{b}^{-1} q$ reduces the seller's gain by $s(\theta)-\delta_{b}^{-1} q$ without improving the chance of a deal. Hence, for any price $q>0$, an

\footnotetext{
${ }^{7}$ Here, I assume that the buyer accepts the offer when he is indifferent. This tie-breaking rule can be justified by the following reasoning. If the indifferent buyer accepts the offer with probability strictly less than one, the seller can be strictly better off by adding an arbitrarily small extra $\varepsilon>0$ to make the buyer accept the offer with probability one. The tie-breaking rule results as this extra $\varepsilon$ vanishes.
} 
optimal security takes the form

$$
s_{q}(\theta)\left\{\begin{array}{l}
=\delta_{b}^{-1} q \text { if } \theta \geq \delta_{b}^{-1} q \\
\in[0, \theta] \quad \text { otherwise }
\end{array} .\right.
$$

A high-level understanding of the flat tail, the common feature of all optimal securities here, is that it minimizes the buyer's incentive to use his information about $\theta$. To see this, consider two states $\theta_{1}$ and $\theta_{2}$ that are greater than $\delta_{b}^{-1} q$ (so that $s\left(\theta_{1}\right)$ and $s\left(\theta_{2}\right)$ could, but need not, equal $\left.\delta_{b}^{-1} q\right)$. The buyer uses his information to differentiate these two states if his probability of acceptance is state-contingent. This happens only when the payoff in one state $s\left(\theta_{1}\right)$ or $s\left(\theta_{2}\right)$ is above $\delta_{b}^{-1} q$ and the other is below it. Suppose $s\left(\theta_{1}\right)>\delta_{b}^{-1} q$ and $s\left(\theta_{2}\right)<\delta_{b}^{-1} q$. By bringing $s\left(\theta_{1}\right)$ down to $\delta_{b}^{-1} q$, the seller reduces the security payoff without sacrificing the chance of a deal. By bringing $s\left(\theta_{2}\right)$ up to $\delta_{b}^{-1} q$, the seller rescues the trade on state $\theta_{2}$ and the gains from it. Therefore, whenever feasible (i.e., $\theta \geq \delta_{b}^{-1} q$ ), the

seller wants to maintain a flat security payoff $\delta_{b}^{-1} q$ to minimize the buyer's incentive to use his information, resulting in a non-state-contingent probability of acceptance for $\theta \geq \delta_{b}^{-1} q$. Although this intuition for the optimality of the flat tail is developed in the special case with exogenous information, it carries over to cases with information acquisition. In that context, since information acquisition is a prerequisite to using information, discouraging the buyer from using his information also discourages him from acquiring information. The optimal security again features a flat tail that minimizes the buyer's incentive to acquire and use information. In Propositions 4 and 5, we will see that introducing information cost also determines the shape of the optimal security for $\theta<\delta_{b}^{-1} q$, making debt uniquely optimal.

Finally, note that, for any $q>0$, the seller's expected payoff is

$$
\operatorname{Pr}\left(\theta \geq \delta_{b}^{-1} q\right) \cdot q\left(1-\delta_{s} \delta_{b}^{-1}\right)
$$

The optimal price $q^{*}$ is pinned down by

$$
q^{*} \in \arg \max _{q \geq 0} \operatorname{Pr}\left(\theta \geq \delta_{b}^{-1} q\right) \cdot q,
$$

and the optimal face value $D^{*}=\delta_{b}^{-1} q^{*}$ follows immediately.

\section{Information Costs and Buyer's Information Acquisi- tion}

Starting from this section, I study the full-fledged model of costly information acquisition. As described above, the seller and the buyer play a sequential-move game. The seller moves first to design the offer $(s, q)$, and the buyer then acquires information about the fundamental 
state according to his (state-contingent) payoff determined by the security. Based on his acquired information, the buyer decides whether to accept the offer. I solve this game with backward induction. In this subsection, I study the buyer's information-acquisition problem for any given offer. I then analyze the seller's security design problem in the following subsections.

The buyer acquires information by arranging to receive a signal, which could be correlated in a fairly arbitrary way to fundamental $\theta$. Specifically, the signal $x$ is drawn from some set $X \subset \mathbb{R}$, according to a conditional probability $\pi(x \mid \theta)$ (i.e., information structure) chosen by the buyer, whose acceptance decision $a$ is then a function of the signal $x$ that is received. ${ }^{8}$ Choosing information structure $\pi$ incurs a cost $c(\pi) \geq 0$. To fix idea, I make several assumptions about the information $\operatorname{cost} c(\cdot)$. The seminal work of Blackwell (1953) introduces a partial ordering to reflect the informativeness of information structures. Structure A is less informative than structure B if A can be obtained from B by the addition of garbling noise. We say that an information cost respects Blackwell's ordering if it assigns a strictly lower cost to strictly less-informative structures in the sense of Blackwell (1953).

Assumption A1: The information cost $c(\cdot)$ strictly respects Blackwell's ordering.

This assumption states that acquiring extra information incurs extra cost. As a result, the buyer will not acquire any useless information. In particular, he always chooses a binarysignal information structure and never mixes between accepting and rejecting the offer upon receiving the signal. Otherwise, he could acquire less information without sacrificing the quality of his acceptance decision. I state this result in the following lemma.

Lemma 1 Let $(\pi, X)$ be the buyer's optimal information structure. Then i) upon receiving any $x \in X$, the buyer strictly prefers either $a=0$ or $a=1$; ii) $X$ contains at most two signal realizations (i.e., $\# X \leq 2$ ).

The proofs of all lemmas and propositions, including this one, are in Appendix B. Here I briefly outline the intuition as follows. First, consider the coarsening of an information structure; that is, combining some signal realizations to form a single realization. This operation adds garbling noise and results in a less costly information structure according to Assumption 1. If the information structure violates i) or ii) in Lemma 1, I show that the buyer can always coarsen his information structure to save the information cost without affecting his probability of acceptance given $\theta$. Regarding $i$ ), if the buyer is indifferent between accepting and rejecting the offer after receiving some signal realization, this realization is useless because it makes the buyer's decision irrelevant to his expected payoff. The buyer can then coarsen the information structure without sacrificing his expected payoff by combining all such realizations with any other signal realization that makes him strictly prefer accepting or rejecting. Regarding ii), if $X$ contains more than two signal realizations, there

\footnotetext{
${ }^{8}$ To be rigorous, the conditional probability $\pi(\cdot \mid \cdot)$ is a mapping from $\mathbb{R}_{+}$, the space of the fundamental state, to $\Delta(X)$, the space of probability measures over $X$.
} 
must be an action taken after receiving at least two signal realizations. Since these realizations lead to the same action, the buyer can combine them to form a single realization and take that action after receiving it. This coarsening operation results in a less-informative information structure and thus a reduction of information cost without affecting the quality of his decision.

As intuitively suggested by Lemma 1 , since the only use of the signal is to make an accept/reject decision, a signal that differentiates more finely among the states just conveys redundant information and thus costs the buyer more resources without improving the quality of his decision. This gives rise to the optimality of a binary information structure, which has two signal realizations, denoted by $\{1,0\}$, interpreted as a yes/no signal as to whether fundamental $\theta$ is good enough to accept. ${ }^{9}$ Consequently, it suffices to focus on informationally efficient strategies defined by these features, and any such strategy can be characterized by a function $m: \mathbb{R}_{+} \rightarrow[0,1]$, where

$$
m(\theta)=\operatorname{Pr}(\text { accepting } \mid \text { state is } \theta) .
$$

Note that the corresponding information structure is given by $\pi(x=1 \mid \theta)=m(\theta)$ and $\pi(x=0 \mid \theta)=1-m(\theta)$, and the associated decision rule is $a=x$, meaning that accepting (rejecting) the offer after receiving $x=1(x=0)$. With a slight abuse of notation but without confusion, I also call $m$ the information structure.

To be rigorous, I require that $m$ belongs to

$$
M=\left\{m \text { Lebesgue measurable }: m(\theta) \in[0,1] \text { for all } \theta \in \mathbb{R}_{+}\right\},
$$

which is the set of all binary information structures, and specify the information cost $c(\cdot)$ over $M$. In particular, let

$$
c(m)=\mathbb{E} g(m(\theta))-g(\mathbb{E} m(\theta))
$$

and $\mu \cdot c(m)$ be the information cost for information structure $m \in M .{ }^{10}$ Here $\mu>0$ controls the difficulty in acquiring information. Larger values of $\mu$ make information acquisition more costly and hence reduce the severity of the adverse selection problem. As $\mu$ goes to infinity, the adverse selection problem vanishes, so that the seller offers the whole underlying asset at price $q=\delta_{b} \cdot \mathbb{E} \theta$, and the buyer accepts the offer without acquiring information. Here

\footnotetext{
${ }^{9}$ Literally, I also need to consider the trivial information structure that has only one signal realization. That is, the buyer does not acquire any information and accepts or rejects the offer unconditionally. This can be viewed as a special case of the binary information structures, as covered by points a) and b) in ii) of Proposition 2.

${ }^{10}$ Here $c(\cdot)$ belongs to the category of posterior separable information costs that are micro-founded by recent literature on information acquisition in decision theory. These information costs are tractable and analytically convenient, due to their additive separability across states. The widely used Shannon's entropy reduction (mutual information) is a special case in this class.
} 
$\mathbb{E}(\cdot)$ is the expectation operator with respect to $\theta$ under prior $P$, and $g:[0,1] \rightarrow \mathbb{R}$ is a function satisfying the following conditions.

Assumption A2: i) $g$ is strictly convex; ii) $g(x)=g(1-x)$; iii) $g$ is third-order differentiable with non-decreasing $x \cdot g^{\prime \prime}(x)$; iv) the left limit $\lim _{x \rightarrow 1} g^{\prime}(x)=\infty$; and v) let $f: \mathbb{R} \rightarrow[0,1]$ be the inverse of $g^{\prime}$ and define $K=\lim _{z \rightarrow \infty} \frac{f^{\prime}(z+1)}{f^{\prime}(z)}$, then $K<1$.

Condition i) is necessary and sufficient to guarantee the non-negativeness of information $\operatorname{cost} c(\cdot)$. The sufficiency follows Jensen's inequality, and the necessity stems from Blackwell's ordering. To see the point of condition ii), note that if I switch the recommendations of the two signal realizations (i.e., "taking action 0 " upon observing signal 1 and "taking action 1" upon observing signal 0), the information content is unchanged and so is the information cost, which requires $c(m)=c(1-m)$. Condition iii) is a technical condition, assumed for the sake of tractability. In particular, the nondecreasing $x \cdot g^{\prime \prime}(x)$ guarantees that the first-order variational approach is sufficient for the main results. Condition iv) states that the (pointwise) marginal cost of avoiding type I and type II errors is infinity whenever there is information acquisition. This point will be further clarified after Proposition 2. For the sake of exposition in Proposition 2, Condition v) defines a constant $K$ to be used to characterize the criteria that sharply distinguish the case with information acquisition from that without it. Note that conditions i) and iii) imply $g^{\prime \prime}>0$ and thus $f^{\prime}>0$, then $K \geq 0$. Condition iv) further implies that $f(z)$ converges to 1 from below as $z$ goes to infinity. If $K>1$, then $f^{\prime}(z)$ is increasing as $z$ goes to infinity and $f(z)$ cannot converge to 1 from below. Hence, conditions i), iii), and iv) imply $K \in[0,1]$ and condition v) simply excludes the non-generic case $K=1$ in which I do not obtain an analytically clear criterion for information acquisition. ${ }^{11}$ For interested readers, I provide more detailed discussions of these conditions in Appendix A.

Example 1: The rational inattention literature employs entropy reduction as a measure of information costs. This measure corresponds to $g(x)=x \ln x+(1-x) \ln (1-x)$ in my setting. This functional form satisfies all the conditions in Assumption A2. In particular, $K=e^{-1}$.

Example 2: Consider a function $g(x)=-x(1-x)$. This function satisfies Conditions i) - iii). It does not satisfy Condition iv) because $g^{\prime}(1)=1<\infty$. Absent Condition iv), Condition v) is not needed, since the domain of $f$ is bounded and there is no need to calculate the limit. This functional form gives rise to information costs with bounded marginal costs of avoiding Type I and Type II errors. I discuss its implications for security design in Subsection 5.2.

An intuitive way to understand $c(m)$ is that it measures the variability of function $m$, the informativeness of the signal with respect to the fundamental state $\theta$. For example, a constant $m(\theta)$ implies $c(m)=0$, because the signal is independent of $\theta$ and thus conveys no

\footnotetext{
${ }^{11}$ Numerical examples suggest that the paper's main qualitative results hold in this knife-edge case.
} 
information about $\theta$. This stems from the strict convexity of function $g$. Hence $c(m)$ is zero if and only if $m(\theta)$ is constant. Therefore, this technology of information acquisition has the attractive property that information acquisition exists if and only if $m(\theta)$ varies over $\theta$, and if and only if the information cost is positive. Also note that the shape (functional form) of $m$ determines not only the quantitative but also the qualitative nature of information. For instance, choosing an $m$ that is constant on some event (i.e., a subset of $\mathbb{R}_{+}$) means that the buyer does not acquire information to distinguish the states within that event, since his decision on that event is not state-contingent. In contrast, the buyer can concentrate his investigation on some event by making $m(\theta)$ highly sensitive to $\theta$ within the event. In this sense, the technology of information acquisition is flexible, because the agent can decide the pointwise quality of his information by choosing any information structure from $M$. I further illustrate this point in the graphic examples in next subsection.

I now examine the buyer's decision problem given any offer $(s, q)$.

\subsection{The Buyer's Decision Problem}

Let $(s, q)$ be an arbitrary offer. Then the buyer's payoff gain from accepting rather than rejecting this offer is

$$
\Delta u(\theta)=\delta_{b} \cdot s(\theta)-q,
$$

which determines his incentive in acquiring information. Specifically, the buyer's payoff gain, conditional on $\theta$, is

$$
\operatorname{Pr}(\text { accepting } \mid \text { state is } \theta) \cdot \Delta u(\theta)+\operatorname{Pr}(\text { rejecting } \mid \text { state is } \theta) \cdot 0=m(\theta) \cdot \Delta u(\theta) \text {. }
$$

Hence his decision problem is

$$
\max _{m \in M} V^{*}(m)=\mathbb{E}[m(\theta) \cdot \Delta u(\theta)]-\mu \cdot c(m)
$$

I present the solution to this decision problem in the following proposition. ${ }^{12}$

Proposition 2 Let $\operatorname{Pr}(\Delta u(\theta) \neq 0)>0$ to exclude the trivial case that the buyer is always indifferent between action 1 and 0 (i.e., accepting or rejecting the offer). Let $m \in M$ be an optimal strategy and

$$
p_{1}=\mathbb{E} m(\theta)
$$

be the corresponding unconditional probability of taking action 1. Then,

i) the optimal strategy is unique;

ii) there are three possibilities for the optimal strategy:

\footnotetext{
${ }^{12}$ Lemma 2 of Woodford (2008) solves a special case of this problem in which the information cost takes the form of entropy reduction.
} 
a) $p_{1}=0$ (i.e., $m(\theta)=0$ almost surely) if and only if

$$
\mathbb{E} K^{-\mu^{-1} \Delta u(\theta)} \leq 1
$$

b) $p_{1}=1$ (i.e., $m(\theta)=1$ almost surely) if and only if

$$
\mathbb{E} K^{\mu^{-1} \Delta u(\theta)} \leq 1
$$

c) $p_{1} \in(0,1)$ if and only if

$$
\mathbb{E} K^{\mu^{-1} \Delta u(\theta)}>1 \text { and } \mathbb{E} K^{-\mu^{-1} \Delta u(\theta)}>1 ;
$$

and in this case, the optimal strategy $m$ is characterized by

$$
\Delta u(\theta)=\mu \cdot\left[g^{\prime}(m(\theta))-g^{\prime}\left(p_{1}\right)\right],
$$

where $K \in[0,1)$ is defined in Assumption A2.

Proposition 2 fully characterizes the buyer's optimal decisions concerning information acquisition. It provides tight criteria that classify the buyer's decisions into three cases. In Case a), the buyer rejects the offer without acquiring information; In Case b), the buyer accepts the offer without acquiring information; In Case c), the buyer acquires information and his decision becomes state-contingent. I first discuss the intuition of the results. I then examine the buyer's posterior beliefs and illustrate flexible information acquisition through numerical examples. The complete proof is in Appendix B.

Suppose the buyer wants to acquire information, then his strategy $m$ cannot be constant, and the resulting unconditional probability $p_{1}$ belongs to $(0,1)$, which is Case $\left.\mathrm{c}\right)$. In particular, he should equate $\Delta u(\theta)$, the pointwise marginal benefit of increasing $m(\theta)$, with $\mu \cdot\left[g^{\prime}(m(\theta))-g^{\prime}\left(p_{1}\right)\right]$, the pointwise marginal information cost of doing so. This results in Equation (8). Intuitively, as $g^{\prime}(m)$ strictly increases in $m$, Equation (8) implies that $m(\theta)$, the probability of accepting the offer conditional on $\theta$, deviates above (below) $p_{1}$, the unconditional probability of acceptance, if $\Delta u(\theta)$ is positive (negative).

To see the role of Condition iv), note that for states such that $\Delta u(\theta)>0$, the buyer should have accepted the offer with probability 1 but mistakenly rejects it with probability $1-m(\theta)$, because the marginal cost of avoiding this Type I error is $\lim _{m(\theta) \rightarrow 1} \mu$. $\left[g^{\prime}(m(\theta))-g^{\prime}\left(p_{1}\right)\right]=\infty$, provided that $p_{1} \in(0,1)$. Similarly, for states such that $\Delta u(\theta)<$ 0 , since $g(m)=g(1-m)$, the marginal cost of avoiding the Type II error is also infinity. Hence, if the buyer acquires information so that the unconditional probability of acceptance 
$p_{1} \in(0,1)$, Condition iv) guarantees an interior solution to the information acquisition problem, that is, $m(\theta) \in(0,1)$ for all $\theta \in \mathbb{R}_{+}$. In contrast, without information acquisition, the buyer either always accepts or always rejects the offer; that is, either $m(\theta)=1$ for all $\theta \in \mathbb{R}_{+}$or $m(\theta)=0$ for all $\theta \in \mathbb{R}_{+}$. We see that Condition iv) helps dichotomize the cases with and without information acquisition. When it fails, the distinction between the cases is blurred. I study this situation in Subsection 5.2.

Moreover, the magnitude of the deviation of $m(\theta)$ from $p_{1}$ is determined by the magnitude of $\Delta u(\theta)$. In doing so, the buyer chooses the shape of the optimal information structure $m(\theta)$ according to the shape of the payoff gain $\Delta u(\theta)$. Consequently, for any states $\theta_{1} \neq \theta_{2}$, $m\left(\theta_{1}\right)-m\left(\theta_{2}\right)$ strictly increases in $\Delta u\left(\theta_{1}\right)-\Delta u\left(\theta_{2}\right)$, and $m\left(\theta_{1}\right)=m\left(\theta_{2}\right)$ if and only if $\Delta u\left(\theta_{1}\right)=\Delta u\left(\theta_{2}\right)$. This reflects the buyer's incentive and ability to differentiate states with different payoffs, which is the essence of flexible information acquisition.

I obtain Inequalities (5), (6), and (7) from comparing the marginal value of information versus its marginal cost. These inequalities provide criteria to determine whether it is worth acquiring information. For example, in Case b) the buyer prefers always accepting the offer to acquiring information if Inequality (6) holds. To appreciate the intuition, consider reducing the payoff gain $\Delta u(\theta)$ by $\varepsilon(\theta)>0$. Since $K<1$, Inequality (6) is more likely to fail after the reduction. This is intuitive because reducing the payoff gain makes the offer less attractive and thus may trigger the buyer to acquire information to screen the offer, rather than accepting it without information acquisition. In addition, according to Jensen's inequality, Inequality (6) is also more likely to fail, if we add some mean preserving spread $\varepsilon(\theta)$ to the payoff gain $\Delta u(\theta)$. Adding this spread may trigger information acquisition because, while $\mathbb{E}[\Delta u(\theta)+\varepsilon(\theta)]=\mathbb{E}[\Delta u(\theta)], \Delta u(\theta)+\varepsilon(\theta)$ is more volatile and information becomes more valuable. The same intuition also applies to Case a). Finally, since $\operatorname{Pr}(\Delta u(\theta) \neq 0)>0$, according to Jensen's inequality, Inequalities (5), (6), and (7) exhaust all possible cases.

These criteria are closely related to Conditions iv) and v), but the derivation is not easy. To avoid distracting readers from the paper's main theme, I outline the derivation of Criteria (5), (6), and (7) from Conditions iv) and v) in Appendix A. I also explore the implications of Condition $g^{\prime}(1)<\infty$, an alternative to Conditions iv) and v), in Subsection 5.2 and show that the paper's qualitative results are robust to this alternative condition.

As mentioned earlier, the optimal strategy $m$ translates to the information structure $\pi(x=1 \mid \theta)=m(\theta)$ and $\pi(x=0 \mid \theta)=1-m(\theta)$, together with the decision rule $a=x$ (i.e., the buyer follows what his signal recommends). To see that the buyer is willing to follow his signal, note that, in Case a), he always receives signal $x=0$, and his posterior is identical to his prior of $\theta$. According to Jensen's inequality, Inequality (5) implies $\mathbb{E} \Delta u(\theta) \leq 0$, and thus the buyer is willing to choose $a=0$ as his signal recommends. Case b) is symmetric to Case a). Now suppose the buyer is ex ante indifferent between accepting and rejecting the 
offer, so that he definitely wants to acquire information and his posterior densities become

$$
\left\{\begin{array}{ll}
\frac{m(\theta) \cdot p(\theta)}{p_{1}} & \text { if } x=1 \\
\frac{[1-m(\theta)] \cdot p(\theta)}{1-p_{1}} & \text { if } x=0
\end{array} .\right.
$$

He is willing to follow the signal if $\mathbb{E}[\Delta u(\theta) \mid x=1] \geq 0$ and $\mathbb{E}[\Delta u(\theta) \mid x=0] \leq 0$, that is, $\mathbb{E}[m(\theta) \Delta u(\theta)] \geq 0$ and $\mathbb{E}([1-m(\theta)] \Delta u(\theta)) \leq 0$. This is true because

$$
\begin{aligned}
& \mathbb{E}[m(\theta) \Delta u(\theta)] \\
= & \mathbb{E}\left[f\left(\mu^{-1} \Delta u(\theta)+g^{\prime}\left(p_{1}\right)\right) \Delta u(\theta)\right] \\
= & \mathbb{E}\left(\left[f\left(\mu^{-1} \Delta u(\theta)+g^{\prime}\left(p_{1}\right)\right)-p_{1}\right] \Delta u(\theta)\right) \\
\geq & 0,
\end{aligned}
$$

where $f$ is the inverse of $g^{\prime}$, defined in Assumption A2. The inequality holds because $f\left(0+g^{\prime}\left(p_{1}\right)\right)=p_{1}$ so that $\left[f\left(\mu^{-1} \Delta u(\theta)+g^{\prime}\left(p_{1}\right)\right)-p_{1}\right]$ and $\Delta u(\theta)$ are always of the same sign. A similar argument verifies that $\mathbb{E}([1-m(\theta)] \Delta u(\theta)) \leq 0$.

Before we examine the security design problem, two numerical examples offer some insight into how the approach of flexible information acquisition works. In the examples, the information cost takes the form of entropy reduction; that is, $g(x)=x \ln x+$ $(1-x) \ln (1-x)$. 


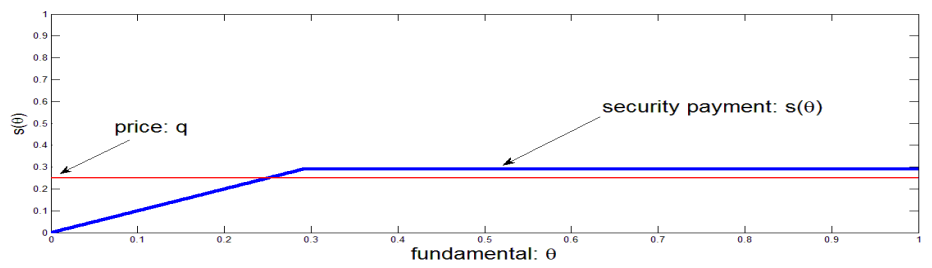

Figure 3.2.1

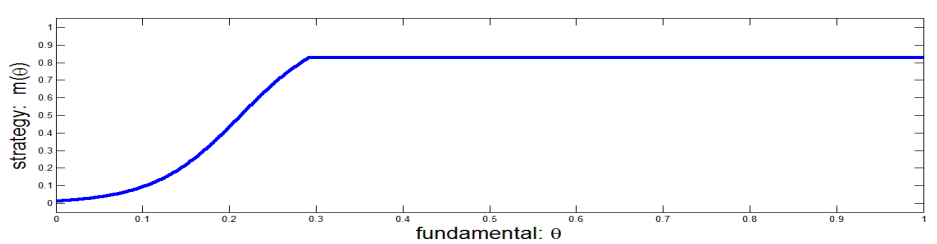

Figure 3.2.2

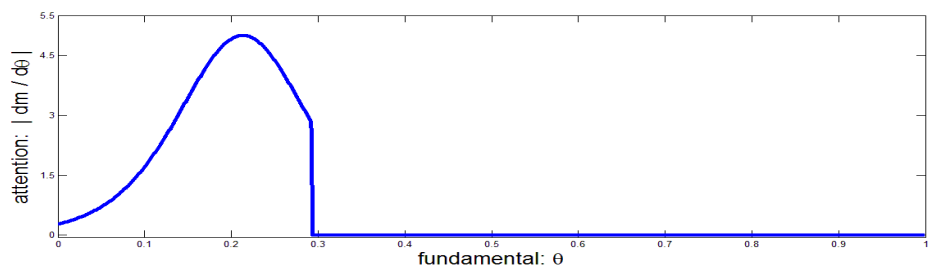

Figure 3.2 .3

Figure 3.2.1 shows the security payoff from a debt security with price $q$. Intuitively, the buyer's ideal strategy is to set $m(\theta)$ equal to the indicator function $1_{\left\{\delta_{b} \cdot s(\theta)-q>0\right\}}$, that is, accepting (rejecting) the offer if the discounted security payoff exceeds (falls below) the price. This strategy is not optimal, however. In the gain region $\left\{\theta: \delta_{b} \cdot s(\theta)-q \geq 0\right\}$, the buyer benefits from reducing $m(\theta)=1$ a little bit, since this operation saves his information cost at a marginal rate of $\mu \cdot\left[g^{\prime}(1)-g^{\prime}\left(p_{1}\right)\right]=\infty$ but just gives up the marginal benefit $\delta_{b} \cdot s(\theta)-q<\infty$. He will keep reducing $m(\theta)$ until the marginal information cost equals the marginal benefit, as stated in Equation (8). A similar argument shows the inferiority of $m(\theta)=0$ in the loss region $\left\{\theta: \delta_{b} \cdot s(\theta)-q<0\right\}$. The resulting optimal strategy is shown in Figure 3.2.2. Since the payoff gain $\delta_{b} \cdot s(\theta)-q$ increases in $\theta$, so does the optimal strategy $m(\theta)$. That is, the buyer arranges to receive a signal that is more likely to suggest accepting the offer at states with relatively higher payoffs, which is intuitive. In particular, $m(\theta)$ is constant across the states above the face value, meaning that no resources are spent on differentiating these states. This is intuitive because the buyer receives constant payoffs in 
all the states above the face value so that he has no incentive to differentiate between these states. More generally, the buyer acquires information to differentiate states in an interval $\left[\theta_{1}, \theta_{2}\right]$ in the sense that $m(\theta)$ varies over $\left[\theta_{1}, \theta_{2}\right]$. Thus $\frac{\left|m\left(\theta_{2}\right)-m\left(\theta_{1}\right)\right|}{\theta_{2}-\theta_{1}}$ measures the average intensity of information acquisition over $\left[\theta_{1}, \theta_{2}\right]$, and as the interval $\left[\theta_{1}, \theta_{2}\right]$ shrinks, the slope $\left|\frac{d m(\theta)}{d \theta}\right|$ reflects the local intensity around $\theta$. The larger the slope, the more sensitive is the buyer's reaction to the change of states around $\theta$, which means that the buyer acquires more information to discern those states. Figure 3.2.3 plots $\left|\frac{d m(\theta)}{d \theta}\right|$, showing that the buyer acquires information intensively for bad states but is inattentive to tail states. This finding is consistent with the well-known fact that debt holders care mainly about default risk.

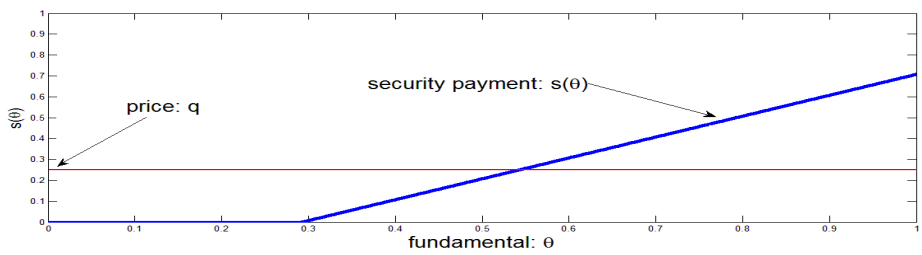

Figure 3.2.4

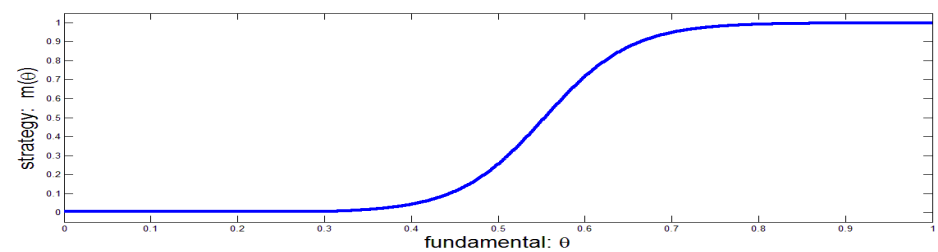

Figure 3.2.5

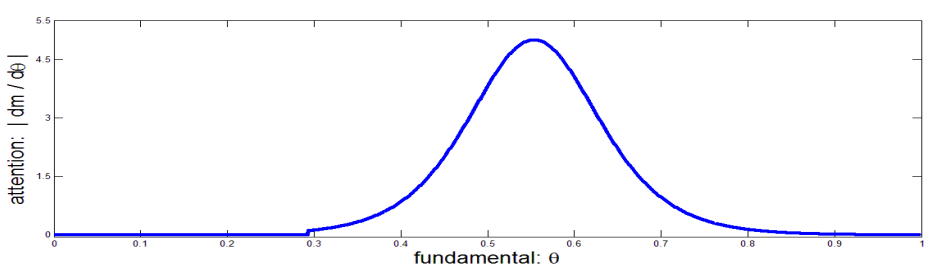

Figure 3.2.6

In contrast, when facing a call option, as shown in Figure 3.2.4, the buyer's optimal strategy $m(\theta)$ is depicted in Figure 3.2.5, which implies that he focuses his information acquisition on good states, where his payoff is state-contingent, as shown in Figure 3.2.6.

These examples clearly show how flexible the incentive to acquire information could be and how it can be captured by the approach of flexible information acquisition. 


\section{Security Design under Flexible Information Acquisi- tion}

Proposition 2 characterizes the buyer's information acquisition against general payoff gains. This section studies the seller's security design problem.

For any offer $(s, q)$, let $m_{s, q} \in M$ denote the buyer's optimal strategy specified by Proposition 2; that is, $m_{s, q}(\theta)$ is the probability that the buyer accepts $(s, q)$ at state $\theta$. Then the seller's expected payoff from proposing $(s, q)$ is

$$
W(s, q)=\mathbb{E}\left(m_{s, q}(\theta) \cdot\left[q-\delta_{s} \cdot s(\theta)\right]\right) .
$$

The seller needs to choose an offer $(s, q)$ to maximize $W(s, q)$. To streamline the analysis, I formally define the equilibrium of this game as follows.

Definition A sequential equilibrium is a collection of the seller's optimal offer $\left(s^{*}, q^{*}\right)$, and $m_{s, q}$, the buyer's optimal information acquisition strategy for any generic offer $(s, q)$, such that

i) $m_{s, q}$ is determined by Proposition 2, that is,

$$
m_{s, q}=\arg \max _{m \in M} \mathbb{E}\left[\left(\delta_{b} \cdot s(\theta)-q\right) \cdot m(\theta)\right]-\mu \cdot c(m)
$$

ii)

$$
\left(s^{*}, q^{*}\right) \in \arg \max _{s(\theta) \in[0, \theta], q \geq 0} \mathbb{E}\left(m_{s, q}(\theta) \cdot\left[q-\delta_{s} \cdot s(\theta)\right]\right) .
$$

According to Proposition 2, three possible cases pertain to the buyer's behavior given the seller's optimal offer. First, the buyer may optimally choose not to acquire any information and accept the seller's offer directly. Second, the buyer may optimally acquire some information induced by the seller's optimal offer and then accept the offer with positive probability (but less than one). Third, the buyer may simply reject the offer without acquiring information. Note that the third case gives the seller an expected payoff of zero and thus corresponds to the seller's outside option of proposing nothing and thereby raising no liquidity. Hence, the seller's individual rationality condition is always satisfied.

In what follows, I first show that, in equilibrium, this last case does not occur, since both the seller and the buyer should have benefited from the trade. Then I characterize the seller's optimal security for the first two cases.

Let $\bar{p}_{s, q}$ denote the buyer's unconditional probability of accepting offer $(s, q)$, that is,

$$
\bar{p}_{s, q}=\mathbb{E} m_{s, q}(\theta)
$$

Proposition $3 \bar{p}_{s^{*}, q^{*}}>0$; that is, trade happens with positive probability. 
Appendix B contains the complete proof. The example presented here provides some intuition. In particular, I show that the seller can always offer a debt security for a wellchosen price, such that the buyer is willing to accept the offer with positive probability and that the seller enjoys a positive expected gain from trade. Consider a debt security $s(\theta)=\min (\theta, D)$ with price

$$
q=-\kappa^{-1} \mu \ln \mathbb{E} \exp \left(-\kappa \mu^{-1} \delta_{b} \min (\theta, D)\right),
$$

where $\kappa=-\ln K>0$. Here, the price $q$ is chosen according to Condition (6), such that the buyer accepts the offer with probability one, that is, $m_{s, q}(\theta)=1$ for all $\theta \in \mathbb{R}_{+}$. Note that

$$
\begin{aligned}
\frac{d q}{d D} & =\delta_{b} \cdot \frac{\mathbb{E}\left[\exp \left(-\kappa \mu^{-1} \delta_{b} \min (\theta, D)\right) \cdot 1_{\{\theta \geq D\}}\right]}{\mathbb{E} \exp \left(-\kappa \mu^{-1} \delta_{b} \min (\theta, D)\right)} \\
& =\delta_{b} \cdot \frac{\operatorname{Pr}(\{\theta \geq D\}) \cdot \exp \left(-\kappa \mu^{-1} \delta_{b} D\right)}{\mathbb{E} \exp \left(-\kappa \mu^{-1} \delta_{b} \min (\theta, D)\right)} \\
& \rightarrow \delta_{b} \text { as } D \rightarrow 0
\end{aligned}
$$

where $1_{\{\theta \geq D\}}$ denotes the indicator function for event $\{\theta \geq D\}$. In addition, $q=0$ when $D=0$. Hence, for face value $D$ close to zero, $q \approx \delta_{b} D>\delta_{s} D$ (since $\left.\delta_{b}>\delta_{s}\right)$. Then the seller's expected payoff is

$$
\begin{aligned}
W(s, q) & =\mathbb{E}\left(m_{s, q}(\theta) \cdot\left[q-\delta_{s} \cdot \min (\theta, D)\right]\right) \\
& \geq \mathbb{E}\left(1 \cdot\left[q-\delta_{s} \cdot D\right]\right) \\
& =q-\delta_{s} \cdot D>0 .
\end{aligned}
$$

The seller's optimal security should be no worse than the debt security constructed above, so that it also necessarily generates a positive expected payoff, which can be achieved only through a successful transaction. The above example does not involve information acquisition. Alternatively, as shown in the proof in Appendix B, it is also possible to construct a debt security that triggers information acquisition but still generates a positive expected payoff to the seller. Both examples suggest that trade happens with positive chance. Despite the (threat of) adverse selection caused by (potential) information acquisition, the seller always prefers to trade, because she can minimize the adverse effect by designing the right security and thus obtain the gains from trade.

According to Proposition 3, I only need to consider Case i): $\bar{p}_{s^{*}, q^{*}}=1$, in which the buyer does not acquire information and always accepts the offer, and Case ii): $\bar{p}_{s^{*}, q^{*}} \in(0,1)$, in which the buyer does acquire information and accepts the offer probabilistically. In Subsection 4.1, I show that the optimal security that does not induce information acquisition (i.e., Case i) is a debt security; in Subsection 4.2, I show that the optimal security that 
induces information acquisition (Case ii) is also a debt security. Therefore, the optimal security is always a debt security.

\subsection{Optimal Security That Deters Information Acquisition}

Consider the case in which the seller's optimal offer is accepted by the buyer without information acquisition. Concretely, this means $\operatorname{Pr}\left(m_{s, q}(\theta)=1\right)=1$. I first consider the buyer's information acquisition problem, given the seller's offer. Then I characterize the seller's optimal security.

According to Proposition 2 and Condition (6), any offer $(s, q)$ that is accepted by the buyer without information acquisition satisfies

$$
\mathbb{E} \exp \left(-\kappa \mu^{-1}\left[\delta_{b} \cdot s(\theta)-q\right]\right) \leq 1
$$

If this inequality holds strictly, the seller could always raise price $q$ to increase her expected payoff, leaving the buyer's response unchanged. Hence, in equilibrium, this inequality must bind; that is,

$$
q=-\kappa^{-1} \mu \ln \mathbb{E} \exp \left(-\kappa \mu^{-1} \delta_{b} \cdot s(\theta)\right)
$$

I show that for any given price $q$, if $s_{q}$ is the optimal security that deters information acquisition, then $s_{q}$ must be a debt security. That is, $s_{q}$ achieves its maximum whenever the limited liability constraint does not bind. Formally, $s_{q}(\theta)<\theta$ implies $s_{q}(\theta) \geq s_{q}\left(\theta^{\prime}\right)$ for all $\theta^{\prime} \in \mathbb{R}_{+}$. Suppose this is not true. Then there exist $\theta_{1}$ and $\theta_{2}$ such that $s_{q}\left(\theta_{2}\right)<\theta_{2}$ and $s_{q}\left(\theta_{2}\right)<s_{q}\left(\theta_{1}\right)$. I will perturb $s_{q}$ to construct a new security, such that the buyer is still willing to purchase the new security at the original price $q$ without acquiring information, and that the expected security payoff from the seller is reduced. In particular, consider slightly perturbing $s_{q}$ by subtracting an infinitesimal $\varepsilon_{1}>0$ from $s_{q}\left(\theta_{1}\right)$ and adding an infinitesimal $\varepsilon_{2}>0$ to $s_{q}\left(\theta_{2}\right)$, respectively. This perturbation is feasible since $s_{q}\left(\theta_{2}\right)<\theta_{2}$ and $s_{q}\left(\theta_{1}\right)>s_{q}\left(\theta_{2}\right) \geq 0$. According to Equation (10), if $\varepsilon_{1}$ and $\varepsilon_{2}$ are chosen such that

$$
\exp \left(-\kappa \mu^{-1} \delta_{b} s_{q}\left(\theta_{2}\right)\right) p\left(\theta_{2}\right) \varepsilon_{2}=\exp \left(-\kappa \mu^{-1} \delta_{b} s_{q}\left(\theta_{1}\right)\right) p\left(\theta_{1}\right) \varepsilon_{1}
$$

the marginal change of the price is zero. ${ }^{13}$ Here, $\kappa=-\ln K$, and $p\left(\theta_{1}\right)$ and $p\left(\theta_{2}\right)$ are the probability densities of the prior distribution at $\theta_{1}$ and $\theta_{2}$, respectively. The marginal

\footnotetext{
${ }^{13}$ The marginal change in price is obtained by taking the first-order derivative of (10) with respect to $s(\theta)$.
} 
change of the expected security payoff to the buyer becomes

$$
\begin{aligned}
& p\left(\theta_{2}\right) \varepsilon_{2}-p\left(\theta_{1}\right) \varepsilon_{1} \\
= & {\left[1-\frac{\exp \left(-\kappa \mu^{-1} \delta_{b} s_{q}\left(\theta_{2}\right)\right)}{\exp \left(-\kappa \mu^{-1} \delta_{b} s_{q}\left(\theta_{1}\right)\right)}\right] p\left(\theta_{2}\right) \varepsilon_{2} } \\
< & 0,
\end{aligned}
$$

where the inequality follows $s_{q}\left(\theta_{2}\right)<s_{q}\left(\theta_{1}\right)$. Hence this perturbation benefits the seller, as it reduces the expected security payoff to the buyer without affecting the price. This contradicts the optimality of $s_{q}$ and thus my claim is true. Let $D(q)$ denote the maximum of $s_{q}(\theta)$. My claim implies that $s_{q}(\theta)=D(q)$ for all $\theta$ such that $s_{q}(\theta)<\theta$ and $s_{q}(\theta) \leq D(q)$ for all $\theta$ such that $s_{q}(\theta)=\theta$. It immediately follows that $s_{q}(\theta)=\min (\theta, D(q))$. That is, for a given price $q$, the optimal security that does not induce information acquisition is a debt security with face value $D(q)$.

I have shown that the optimal security $s_{q}$ given any price $q$ is debt. Now I characterize the optimal price $q^{*}$, or equivalently, the optimal face value $D^{*}$. Since the offer is always accepted in this equilibrium, the seller's expected payoff $W(s, q)$ becomes

$$
\begin{aligned}
& \mathbb{E}\left[q-\delta_{s} \cdot s(\theta)\right] \\
= & -\kappa^{-1} \mu \ln \mathbb{E} \exp \left(-\kappa \mu^{-1} \delta_{b} \cdot \min (\theta, D)\right)-\delta_{s} \cdot \mathbb{E} \min (\theta, D) .
\end{aligned}
$$

Note that the expected payoff becomes zero when $D=0$. Proposition 3 then implies the optimal face value $D^{*}$ is strictly positive. Hence the first order condition with respect to $D$ leads to

$$
\frac{\delta_{b} \cdot \exp \left(-\kappa \mu^{-1} \delta_{b} D^{*}\right)}{\mathbb{E} \exp \left(-\kappa \mu^{-1} \delta_{b} \cdot \min \left(\theta, D^{*}\right)\right)}-\delta_{s}=0 .
$$

The optimal face value $D^{*}$ results from the following trade-off. On one hand, increasing $D$ increases the price $q$ that the buyer is willing to pay without acquiring information. This is captured by the first term on the left-hand side of Equation (11). On the other hand, increasing $D$ mechanically increases the expected security payoff from the seller, as captured by the second term on the left-hand side of (11). The optimal face value $D^{*}$ balances these two effects as in (11). According to Equation (10), I can express the expectation in Equation (11) in terms of the optimal price $q^{*}$ and simplify (11) to

$$
D^{*}=\kappa^{-1} \mu \delta_{b}^{-1} \cdot\left[\ln \delta_{b}-\ln \delta_{s}\right]+\delta_{b}^{-1} q^{*} .
$$

Equation (10) also requires that

$$
q^{*}=-\kappa^{-1} \mu \ln \mathbb{E} \exp \left(-\kappa \mu^{-1} \delta_{b} \cdot \min \left(\theta, D^{*}\right)\right) .
$$


The optimal face value $D^{*}$ and price $q^{*}$ can be pinned down from the above two equations. I summarize these results in Proposition 4, with the formal proof relegated to Appendix B.

Proposition 4 For any given price $q>0$, if $s_{q}$ is the seller's optimal security that induces the buyer to accept $\left(s_{q}, q\right)$ without acquiring information, then $s_{q}$ must be a debt security. Moreover, define

$$
\begin{aligned}
D^{*}(q) & =\kappa^{-1} \mu \delta_{b}^{-1} \cdot\left[\ln \delta_{b}-\ln \delta_{s}\right]+\delta_{b}^{-1} q \\
\text { and } \quad h(q) & =-\kappa^{-1} \mu \ln \mathbb{E} \exp \left(-\kappa \mu^{-1} \delta_{b} \cdot \min \left(\theta, D^{*}(q)\right)\right)
\end{aligned}
$$

If $\left(s^{*}, q^{*}\right)$ is the optimal offer accepted by the buyer without acquiring information, then $q^{*}>0$ and is the unique fixed point of $h(\cdot)$ on $\mathbb{R}_{+}$, and $s^{*}(\theta)=\min \left(\theta, D^{*}\left(q^{*}\right)\right)$.

The main insight is that once the security payoff differs across states, say $s_{q}\left(\theta_{1}\right)>s_{q}\left(\theta_{2}\right)$, the seller could benefit from reducing $s_{q}\left(\theta_{1}\right)$ and increasing $s_{q}\left(\theta_{2}\right)$ until $s_{q}\left(\theta_{1}\right)=s_{q}\left(\theta_{2}\right)$. In other words, the seller would prefer a flat security whenever it is feasible. Non-flat securities give the buyer an incentive to acquire information to differentiate states with a different security payoff. The seller then has to reduce the price to discourage the buyer from doing so. Flat securities eliminate such an incentive for the buyer and thus allow the seller to charge more. Because of the limited liability constraint, however, the only security that is completely flat is $s(\theta)=0$ for all $\theta \in \mathbb{R}_{+}$, which forgoes all of the gains from trade. As a compromise, debt securities with a positive face value capture some gains from trade and are flat whenever feasible.

The intuition of the optimality of the flat tail developed in the special case of zero information cost in Subsection 2.1 carries over here. The flat tail renders information that helps distinguish between states associated with the tail useless to the buyer. The addition here is endogenous information acquisition. Since such information is now costly to acquire, the flat tail deters the buyer from acquiring it and thus mitigates the resulting adverse selection, while in the special case, the buyer already possesses information, and the flat tail simply discourages its use.

In both cases, the seller wants to maintain a flat security payoff whenever possible. However, depending on whether information is costly, the optimal securities behave differently in states where the cash flow $\theta$ falls short of the face value. There, the security payoff must be state contingent. When it is costless to discern those states, the offer is rejected in all those states. The difference between the security payoff and the face value does not matter, and the optimal security does not have to feature a 45-degree-line portion. However, when information acquisition is costly, then the greater the difference between the security payoff and the face value, the greater the buyer's incentive to acquire information. To deter the buyer from acquiring information, the seller should not only make the security payoff as flat as possible for states in which $\theta$ is above the face value, but also minimize the difference 
between the security payoff and the face value for states in which $\theta$ is below the face value. This results in the binding limited liability constraint, and the optimal security features a 45-degree-line portion.

The price $q^{*}$ and face value $D^{*}$ are determined so that the buyer breaks even between acquiring and not acquiring information. In particular, equation (10) implies

$$
\begin{aligned}
q^{*} & =-\kappa^{-1} \mu \ln \mathbb{E} \exp \left(-\kappa \mu^{-1} \delta_{b} \cdot \min \left(\theta, D^{*}\right)\right) \\
& <\delta_{b} \cdot \mathbb{E} \min \left(\theta, D^{*}\right)
\end{aligned}
$$

where the inequality follows Jensen's inequality. Since the offer induces no information acquisition, both parties remain symmetrically uninformed, and the seller should have charged $\delta_{b} \cdot \mathbb{E} \min \left(\theta, D^{*}\right)$. However, the seller finds it optimal to charge a lower price $q^{*}$ in order to induce the buyer not to acquire information.

\subsection{Optimal Security with Information Acquisition}

The previous subsection establishes that, for any given price, debt is the optimal security that does not induce information acquisition. This subsection shows that, for any given price, the optimal security that does induce the buyer to acquire information is also debt. Therefore, I can conclude that, for any given price, the optimal security is debt whether or not it triggers information acquisition.

Since the buyer acquires information, Proposition 2 and condition (8) prescribe that $m_{s, q}$, the buyer's response to an arbitrary offer $(s, q)$, is given by

$$
\delta_{b} \cdot s(\theta)-q=\mu \cdot\left[g^{\prime}\left(m_{s, q}(\theta)\right)-g^{\prime}\left(\bar{p}_{s, q}\right)\right]
$$

where

$$
\bar{p}_{s, q}=\mathbb{E} m_{s, q}(\theta)
$$

is the buyer's unconditional probability of accepting the offer. Taking into account the buyer's response $m_{s, q}$, the seller's problem is to choose $(s, q)$ to maximize her expected payoff,

$$
W(s, q)=\mathbb{E}\left(m_{s, q}(\theta) \cdot\left[q-\delta_{s} \cdot s(\theta)\right]\right),
$$

subject to the limited liability constraint,

$$
s(\theta) \in[0, \theta] .
$$

As indicated in the objective function (13), the seller wants a high probability of acceptance $m_{s, q}(\theta)$ and a large profit $q-\delta_{s} \cdot s(\theta)$. Equation (12), however, imposes a trade-off between these two goals in security design - a higher $m_{s, q}(\theta)$ requires a larger $s(\theta)$ and thus reduces 
the seller's profit at state $\theta$.

I show that, for any given price $q$, if $s_{q}$ is the optimal security that induces information acquisition, $s_{q}$ must be a debt security. Analogous to Subsection 4.1, I use a perturbation argument to show that the optimality of $s_{q}$ requires that it achieves its maximum, whenever the limited liability constraint is slack. That is, $s_{q}(\theta)<\theta$ implies $s_{q}(\theta) \geq s_{q}\left(\theta^{\prime}\right)$ for all $\theta^{\prime} \in \mathbb{R}_{+}$. If this is not true, then there exist $\theta_{1}$ and $\theta_{2}$ such that $s_{q}\left(\theta_{2}\right)<\theta_{2}$ and $s_{q}\left(\theta_{2}\right)<s_{q}\left(\theta_{1}\right)$. I can perturb $s_{q}$ to construct a new security, such that the buyer still acquires information and accepts the new security at the original price $q$ probabilistically, and that the seller enjoys a strictly higher expected payoff. Consider slightly perturbing $s_{q}$ by subtracting an infinitesimal $\varepsilon_{1}>0$ from $s_{q}\left(\theta_{1}\right)$ and adding an infinitesimal $\varepsilon_{2}>0$ to $s_{q}\left(\theta_{2}\right)$, respectively. This perturbation is feasible since $s_{q}\left(\theta_{2}\right)<\theta_{2}$ and $s_{q}\left(\theta_{1}\right)>s_{q}\left(\theta_{2}\right) \geq 0$. According to Equation (12), the buyer's conditional probability of acceptance at $\theta$ can be expressed as

$$
m_{s, q}(\theta)=f\left(\mu^{-1}\left[\delta_{b} s_{q}(\theta)-q\right]+g^{\prime}\left(\bar{p}_{s, q}\right)\right),
$$

where $f$ is the inverse function of $g^{\prime}$ defined in Condition v) of Assumption A2. Note that, if the unconditional probability of acceptance $\bar{p}_{s, q}$ does not change, adding an infinitesimal $\varepsilon$ to $s_{q}(\theta)$ results in a marginal change of $m_{s, q}(\theta)$ equal to $\mu^{-1} \delta_{b} f^{\prime}\left(\mu^{-1}\left[\delta_{b} s_{q}(\theta)-q\right]+g^{\prime}\left(\bar{p}_{s, q}\right)\right) \varepsilon$. Hence, if $\varepsilon_{1}$ and $\varepsilon_{2}$ are chosen such that

$$
f^{\prime}\left(\mu^{-1}\left[\delta_{b} s_{q}\left(\theta_{2}\right)-q\right]+g^{\prime}\left(\bar{p}_{s, q}\right)\right) p\left(\theta_{2}\right) \varepsilon_{2}=f^{\prime}\left(\mu^{-1}\left[\delta_{b} s_{q}\left(\theta_{1}\right)-q\right]+g^{\prime}\left(\bar{p}_{s, q}\right)\right) p\left(\theta_{1}\right) \varepsilon_{1}
$$

the marginal change in $\bar{p}_{s, q}$ is zero, which further guarantees that the perturbation does not change $m_{s, q}(\theta)$ for $\theta \neq \theta_{1}$ or $\theta_{2}$. To simplify the exposition, in the rest of this section, I use $f_{i}^{\prime}$ to denote $f^{\prime}\left(\mu^{-1}\left[\delta_{b} s_{q}\left(\theta_{i}\right)-q\right]+g^{\prime}\left(\bar{p}_{s, q}\right)\right)$ for $i \in\{1,2\}$.

Now I examine the marginal impact of this perturbation on the seller's expected payoff (13). This marginal impact equals

$$
\begin{aligned}
& \delta_{s}\left[m_{s, q}\left(\theta_{1}\right) \cdot p\left(\theta_{1}\right) \varepsilon_{1}-m_{s, q}\left(\theta_{2}\right) \cdot p\left(\theta_{2}\right) \varepsilon_{2}\right] \\
& +\mu^{-1} \delta_{b}\left(\left[q-\delta_{s} s_{q}\left(\theta_{2}\right)\right] \cdot f_{2}^{\prime} p\left(\theta_{2}\right) \varepsilon_{2}-\left[q-\delta_{s} s_{q}\left(\theta_{1}\right)\right] \cdot f_{1}^{\prime} p\left(\theta_{1}\right) \varepsilon_{1}\right) .
\end{aligned}
$$

The first term captures the direct effect of subtracting $\varepsilon_{1}$ from $s_{q}\left(\theta_{1}\right)$ and adding $\varepsilon_{2}$ to $s_{q}\left(\theta_{2}\right)$, disregarding the induced variation of $m_{s, q}\left(\theta_{1}\right)$ and $m_{s, q}\left(\theta_{2}\right)$. The second term measures the indirect effect of subtracting $\varepsilon_{1}$ from $s_{q}\left(\theta_{1}\right)$ and adding $\varepsilon_{2}$ to $s_{q}\left(\theta_{2}\right)$ through decreasing $m_{s, q}\left(\theta_{1}\right)$ and increasing $m_{s, q}\left(\theta_{2}\right)$, respectively. I omit some higher order infinitesimal that stems from the interaction of the direct and indirect effects.

To see that the seller benefits from the perturbation, I show that the direct and the indirect effects are jointly positive. Note that the perturbation is constructed according to Equation (15) such that the (probability weighted) marginal decrease of $m_{s, q}\left(\theta_{1}\right)$ and 
increase of $m_{s, q}\left(\theta_{2}\right)$ are equal. Thus the indirect effect is proportional to

$$
\begin{aligned}
& {\left[q-\delta_{s} s_{q}\left(\theta_{2}\right)\right]-\left[q-\delta_{s} s_{q}\left(\theta_{1}\right)\right] } \\
= & \delta_{s}\left[s_{q}\left(\theta_{1}\right)-s_{q}\left(\theta_{2}\right)\right] \\
> & 0
\end{aligned}
$$

so that the indirect effect is strictly positive. Equation (15) also implies that the direct effect is proportional to

$$
\begin{aligned}
& \frac{m_{s, q}\left(\theta_{1}\right)}{f_{1}^{\prime}}-\frac{m_{s, q}\left(\theta_{2}\right)}{f_{2}^{\prime}} \\
= & m_{s, q}\left(\theta_{1}\right) \cdot g^{\prime \prime}\left(m_{s, q}\left(\theta_{1}\right)\right)-m_{s, q}\left(\theta_{2}\right) \cdot g^{\prime \prime}\left(m_{s, q}\left(\theta_{2}\right)\right),
\end{aligned}
$$

where the equality follows the fact that $g^{\prime \prime}=1 / f^{\prime}$. According to Equation (12), $s_{q}\left(\theta_{1}\right)>$ $s_{q}\left(\theta_{2}\right)$ implies $m_{s, q}\left(\theta_{1}\right)>m_{s, q}\left(\theta_{2}\right)$. It immediately follows that the direct effect is nonnegative, as $x \cdot g^{\prime \prime}(x)$ is non-decreasing. ${ }^{14}$ The perturbation makes the seller strictly better off, contradicting the optimality of $s_{q}$. Hence, $s_{q}$ achieves its maximum whenever $s_{q}(\theta)<\theta$. Let $D(q)$ denote that maximum. I obtain $s_{q}(\theta)=D(q)$ for all $\theta$ such that $s_{q}(\theta)<\theta$, and $s_{q}(\theta) \leq D(q)$ for all $\theta$ such that $s_{q}(\theta)=\theta$. It immediately follows that $s_{q}(\theta)=$ $\min (\theta, D(q))$ is a debt security. I summarize these results in Proposition 5, with the formal proof relegated to Appendix B.

Proposition 5 For any price $q>0$, if $s_{q}$ is the seller's optimal security that induces the buyer to acquire information, then $s_{q}$ must be a debt security.

This proposition states that, even if the optimal security triggers information acquisition, it must be a debt security. The results follow from a perturbation argument that is in the same spirit as that for the case without information acquisition but that is more delicately constructed to cope with the interaction between security design and information acquisition. In particular, whenever the security payoff differs, say $s_{q}\left(\theta_{1}\right)>s_{q}\left(\theta_{2}\right)$, the buyer acquires information that enables him to differentiate between $\theta_{1}$ and $\theta_{2}$. That is, he will be able to accept the offer with higher probability at state $\theta_{1}$ than at $\theta_{2}$. This adverse selection harms the seller, who then wants to make the security payoff flat whenever possible. As in the special case with zero information cost in Subsection 2.1, the flat security payoff helps discourage the buyer's use of information that differentiates states with the same security payoff and thus also discourage the acquisition of such information. This gives rise to the flat tail portion of debt.

\footnotetext{
${ }^{14}$ This is the only step of the derivation that involves Condition iii) of Assumption A2. Since the indirect effect is strictly positive, Condition iii) is not necessary and could be relaxed. I stick to this condition for the sake of transparency, since relaxing it does not deliver new insights but complicates the analysis.
} 
When the cash flow $\theta$ is too low to support the flat security payoff, the limited liability constraint binds and gives rise to the 45-degree-line portion of the debt security. This is because, due to the flexible nature of information acquisition, the conditional probability of acceptance $m(\theta)$ at any such state $\theta$ is strictly positive and would decrease in response to any downward deviation of the security payoff from $\theta$ (the 45 -degree line). This differs from the special case with zero information cost in Subsection 2.1. There, for any state $\theta$, the conditional probability of acceptance $m(\theta)=0$ and thus cannot decrease further to respond to any downward deviation of the security payoff from the 45-degree line. Thus, the limited liability constraint does not have to bind.

The flat tail of debt securities reflects the seller's incentive to minimize the buyer's information acquisition. This incentive stems from an important feature of my model: fixed aggregate cash flow. That is, the aggregate cash flow $\theta$ to be split between the seller and the buyer is invariant to the design of the security and to the success or failure of the transaction. This generates a conflict of interest between the two parties. That is, the buyer gains at the expense of the seller through endogenous adverse selection. Specifically, the buyer can acquire information that enables him to reject an offer when the security payoff is lower than the price and accept it otherwise. The acquisition of such information does not affect the aggregate cash flow and thus causes a deadweight loss in the form of information cost. A debt security not only saves the buyer's information cost but also mitigates the potential loss caused by the adverse selection, and thus enhances liquidity provision.

Propositions 4 and 5 jointly establish the optimality of debt securities regardless of whether the buyer's information acquisition is triggered. Unfortunately, due to the complexity of functional analysis, there is not an intuitive and closed-form characterization of the optimal face value $D^{*}$ of the optimal debt security and its price $q^{*}$. For interested readers, I characterize them implicitly by a set of equations in the appendix and conduct numerical comparative static analysis in the next subsection.

\subsection{Comparative Statics}

So far, I have been focusing on qualitative analysis and have established the optimality of debt. In fact, my model not only predicts debt as the uniquely optimal security regardless of parameter values, but also sheds light on the determination of the face value $D^{*}$, price $q^{*}$, and interest rate $D^{*} / q^{*}-1$ of the optimal debt security. First, I conduct comparative static analysis on how information cost $\mu$ affects these variables. Here I reinterpret $\mu$ as the opaqueness/complexity of the underlying asset to the buyers. The comparative statics then provide implications of asset opaqueness/complexity for security design, which turn out to be different in booms and busts. Here booms (busts) are captured by a prior distribution of $\theta$ with high (low) mean and low (high) variance. In particular, I calculate the comparative statics under prior distributions $N(0.8,0.2)$ and $N(0.6,0.3)$, both truncated on the interval 
$[0$, mean +2 standard deviations $]$. The information cost is assumed to take the form of entropy reduction, i.e., $g=x \ln x+(1-x) \ln (1-x)$, which satisfies all the conditions in Assumption A2. The results are shown in Figures 4.3 .1 and 4.3.2, respectively.

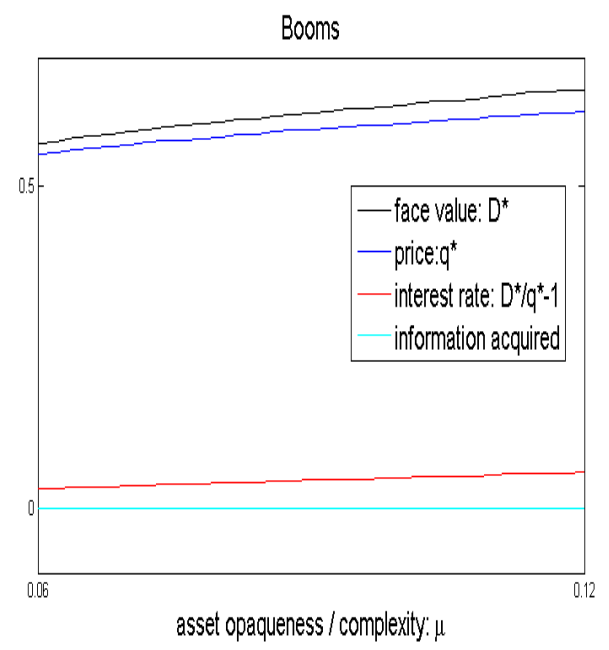

Figure 4.3.1

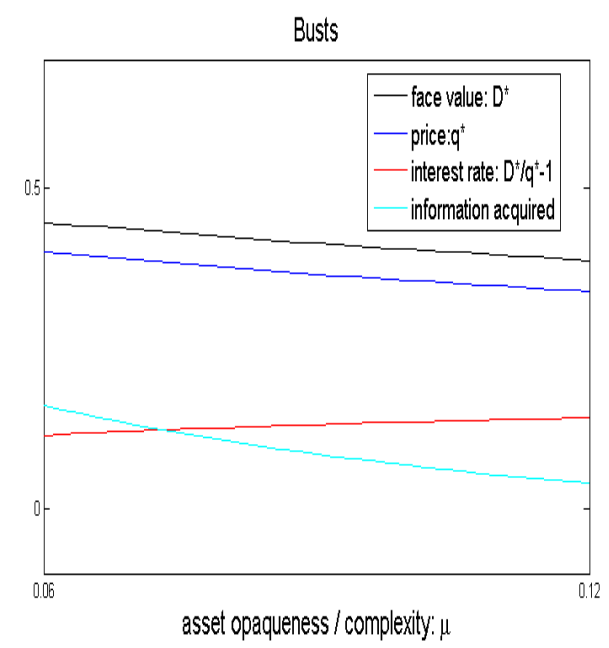

Figure 4.3 .2

Featuring high expected cash flows and low uncertainty, Figure 4.3.1 represents booms. There, the buyer chooses not to acquire information, as depicted by the light blue line. Given that, as asset opaqueness/complexity increases, the seller can sell a bigger chunk of the asset (as indicated by the increasing face value shown by the black line) for a higher price (shown by the blue line). In contrast, featuring low expected cash flows and high uncertainty, Figure 4.3.2 represents busts. High uncertainty prompts the buyer to acquire information, and the amount decreases in asset opaqueness/complexity, as depicted by the light blue line. In busts, the buyer is less willing to buy securities backed by assets with higher opaqueness/complexity, resulting in a decrease in the optimal face value and price. It is also intuitive that, for any given level of opaqueness/complexity, the seller can sell a bigger chunk of the asset (i.e., higher face value) for a higher price in booms than in busts. ${ }^{15}$

Despite the sharp contrast in the boom and bust behavior of these three variables, an increase in opaqueness/complexity always leads to an increase in the interest rate of debt, as shown by the red lines. This is because opaqueness reduces the buyer's informational advantage, and a higher interest rate is demanded to compensate.

I next present comparative statics of $D^{*}, q^{*}$ and interest rate $D^{*} / q^{*}-1$ with respect to the seller's discount factor $\delta_{s}$ under the same prior distributions and information cost,

\footnotetext{
${ }^{15}$ This boom and bust argument is conceptually related to the comparison of a security's information sensitivities in different economic situations in Dang, Gorton, and Hölmström (2015).
} 
as shown in Figures 4.3.3 and 4.3.4, respectively. In both booms and busts, as the seller becomes more patient, the gains from trade shrink, resulting in less trade as reflected by the lower face value and price. Intuitively, the interest rate decreases in $\delta_{s}$, since a more patient seller is less willing to make attractive offers. Figure 4.3.3 represents booms, featuring low uncertainty and high expected cash flows. The buyer thus chooses not to acquire any information, as depicted by the light blue line. In contrast, in bust times, represented by Figure 4.3.4, high uncertainty prompts the buyer to acquire information, and the amount decreases in $\delta_{s}$ since information becomes less valuable with less gains from trade. It is also intuitive that for any given level of $\delta_{s}$, the seller can sell a bigger chunk of the asset (i.e., higher face value) for a higher price in booms than in busts.

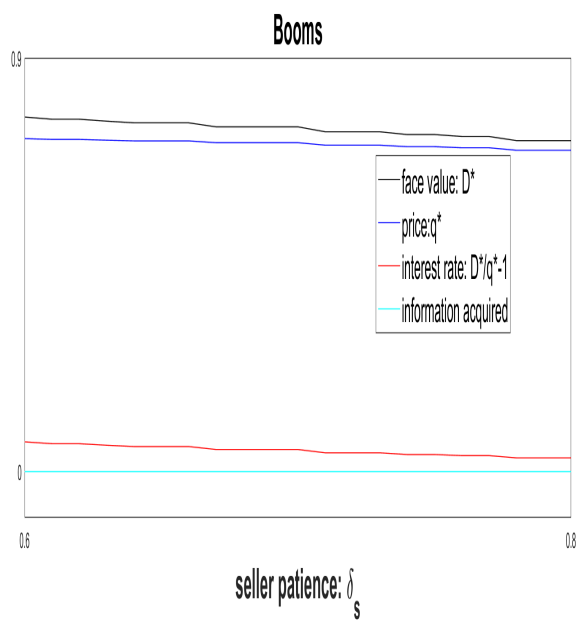

Figure 4.3 .3

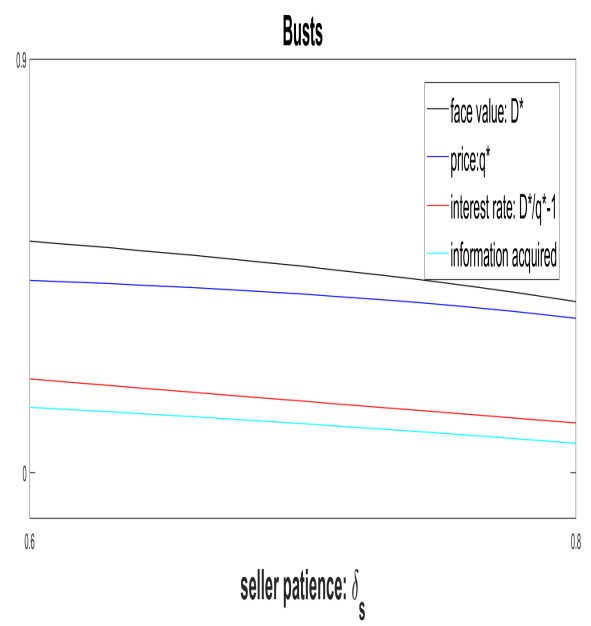

Figure 4.3.4

\section{Discussion of Model Variations}

In this section several variants of the baseline model are analyzed. The first shows that debt is no longer optimal if the aggregate cash flow is not exogenously fixed. The next examines the implications of information costs with bounded marginal cost of avoiding Type I and Type II errors. I show that the optimal security remains a debt security whenever trade happens. I then show that my model can be extended to cover the case with multiple underlying assets, in which pooling the assets and issuing a senior tranche is optimal. Finally, I discuss the case of Bertrand competition between multiple buyers. I argue that competitive bidding with information acquisition changes the nature of the problem and that debt may not be optimal. 


\subsection{Variable Aggregate Cash Flow}

As discussed in the remarks of Proposition 5, fixed aggregate cash flow is the key feature of the baseline model that leads to the optimality of debt. To further clarify this point, I consider a simple model variation with variable aggregate cash flow.

In this variant, the seller has exclusive access to a project that can generate future cash flow $\theta$ distributed according to prior $P$. To initiate this project, the seller needs to raise capital $k=\mathbb{E}(\theta)$ from the buyer. As before, the seller first proposes a security $s$ to the buyer in exchange for capital $k$. The buyer then acquires information and decides whether to accept the offer $(s, k)$. The project gets financed and generates future cash flow $\theta$ only if the buyer accepts the offer. Hence, the aggregate cash flow now depends on the success of the transaction.

To see the difference between the baseline model and this variant, note that the former resembles an exchange economy, where social surplus stems from reallocating some cash flow from the impatient seller to the less impatient buyer. In contrast, this variant features a production economy, where cash flows can be generated only if the transaction succeeds. To highlight this production feature, I assume $\delta_{s}=\delta_{b}=1$ to eliminate the benefit of reallocation. Moreover, since the project's ex ante net present value (NPV) is $\mathbb{E}(\theta)-k=0$, no surplus can be created without information acquisition. Under this setting, I obtain the following formal result. ${ }^{16}$

Proposition 6 Let $s^{*}$ be the optimal security. Then i) the project is financed with positive probability; ii) there exists a $D^{*}$, such that $s^{*}(\theta)=\theta$ when $\theta \leq D^{*}$; and iii) $s^{*}(\theta)$ strictly increases in $\theta$ and $s^{*}(\theta)<\theta$ when $\theta>D^{*}$.

Proposition 6 shows that debt is not optimal when the aggregate cash flow is variable. Specifically, it articulates in what sense the new optimal security differs from a debt security. When the cash flow is lower than a threshold $D^{*}$, the optimal security still follows the 45-degree line, that is, the limited liability constraint. But as the cash flow rises above threshold $D^{*}$, the optimal security is no longer flat but is strictly increasing. Overall, the optimal security always strictly increases in cash flow and thus induces a strictly increasing probability of transaction.

The deviation of this new optimal security from a debt security when cash flow $\theta$ is higher than threshold $D^{*}$ is intuitive and further clarifies the role of information acquisition in the model. Since the seller and the buyer are equally patient and the ex ante NPV of the project is zero, information acquisition becomes the only source of surplus. Thus, information is potentially valuable, and consequently, the seller designs a security that is no longer flat (as debt) to encourage information acquisition. In my baseline model, information acquisition

\footnotetext{
${ }^{16}$ To make the result as clear as possible, the information cost takes the form of entropy reduction here. Specifically, $g(x)=x \ln x+(1-x) \ln (1-x)$, which satisfies all the conditions in Assumption A2.
} 
always reduces social surplus because it creates adverse selection and lowers the probability of transaction, and therefore debt is optimal for discouraging information acquisition. ${ }^{17}$

Besides the production feature, this model also differs from the baseline model in that $k$, the price of the security, is exogenous, while price $q$ in the baseline model is the seller's choice variable. Since I have shown in the baseline model that the optimal security for any given price $q$ is a debt security, this difference is inessential for the purpose of comparing the shapes of the optimal securities in the two scenarios.

\subsection{Bounded Marginal Cost of Information Acquisition}

To analyze security design with a bounded marginal cost of avoiding Type I and Type II errors, I replace Conditions iv) and v) of Assumption A2 with Condition vi): $g^{\prime}(1)<\infty$. I show that, for any given price $q>0$, the optimal security remains a debt security in the states at which trade happens.

Recall that, when the (pointwise) marginal cost $g^{\prime}$ is unbounded, the solution to the information acquisition problem is either completely interior; that is, $m(\theta) \in(0,1)$ for all $\theta \in \mathbb{R}_{+}$, or completely on the boundary; that is, $m(\theta)=1$ for all $\theta \in \mathbb{R}_{+}$or $m(\theta)=0$ for all $\theta \in \mathbb{R}_{+}$. The interior solution involves information acquisition, while the boundary solutions do not. This distinction is blurred under bounded marginal cost $g^{\prime}$. As a counterpart of Proposition 2, the buyer's decision is now characterized by

$$
\Delta u(\theta)=\mu \cdot\left[g^{\prime}(m(\theta))-g^{\prime}\left(p_{1}\right)\right]+\eta(\theta)
$$

where

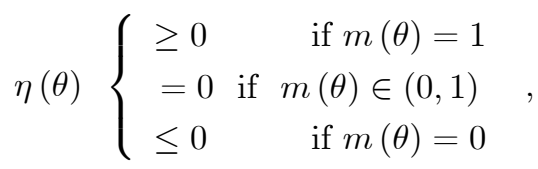

and $p_{1}=\mathbb{E} m(\theta)$ is the unconditional probability of taking action 1 . In particular, when $\Delta u(\theta)$, the marginal benefit of increasing $m(\theta)$, exceeds $\mu \cdot\left[g^{\prime}(1)-g^{\prime}\left(p_{1}\right)\right]$, the highest possible marginal cost, $m(\theta)$ reaches its upper boundary 1 . Similarly, when $\Delta u(\theta)$ falls below $\mu \cdot\left[g^{\prime}(0)-g^{\prime}\left(p_{1}\right)\right], m(\theta)$ reaches its lower boundary 0 .

As before, let $s_{q}$ denote the optimal security for any given price $q>0$. Correspondingly, the state contingent payoff for the buyer is $\delta_{b} \cdot s_{q}(\theta)-q$. It is straightforward to see that the optimality of $s_{q}$ requires $\eta(\theta) \leq 0$ to hold almost surely. Otherwise, if there is some $\theta$ such that $\eta(\theta)>0$, the seller will be strictly better off by reducing the security payoff $s_{q}(\theta)$ a little without sacrificing $m_{s_{q}, q}(\theta)$, the probability of trade at $\theta$, which remains one. ${ }^{18}$

\footnotetext{
${ }^{17}$ In this vein, Yang and Zeng (2018) consider a general production economy and argue that projects of different natures require different means of financing.

${ }^{18}$ This perturbation is feasible because $\delta_{b} \cdot s_{q}(\theta)-q>\mu \cdot\left[g^{\prime}(1)-g^{\prime}\left(p_{1}\right)\right]>0$. This argument cannot be
} 
This observation allows the same perturbation argument used for Proposition 5 to work here. In particular, I claim that in states at which the trade happens with positive probability, $s_{q}$ achieves its maximum whenever the limited liability constraint does not bind. If not, consider $\theta_{1}$ and $\theta_{2}$ such that $s_{q}\left(\theta_{2}\right)<\theta_{2}$ and $s_{q}\left(\theta_{2}\right)<s_{q}\left(\theta_{1}\right)$. Again, I adopt the perturbation used in the argument for Proposition 5; that is, subtracting an infinitesimal $\varepsilon_{1}>0$ from $s_{q}\left(\theta_{1}\right)$ and adding an infinitesimal $\varepsilon_{2}>0$ to $s_{q}\left(\theta_{2}\right)$, respectively. The argument for Proposition 5 may not work if the perturbation does not move $m_{s_{q}, q}\left(\theta_{2}\right)$ or $m_{s_{q}, q}\left(\theta_{1}\right)$. But this possibility is excluded by the previous observation and the condition that trade happens at $\theta_{1}$ and $\theta_{2}$; i.e., both $m_{s_{q}, q}\left(\theta_{1}\right)$ and $m_{s_{q}, q}\left(\theta_{2}\right)$ are positive. Hence, the perturbation argument for Proposition 5 works here, and I establish the following proposition.

Proposition 7 For any price $q>0$, the seller's optimal security $s_{q}$ must be a debt security on all $\theta$ such that $m_{s_{q}, q}(\theta)>0$.

This proposition shares the same intuition as its predecessors in Section 4. The difference is that, depending on the distribution of $\theta$ and the value of $q$, there may exist a non-zero measure set of $\theta$ such that trade does not happen; that is, $m_{s_{q}, q}(\theta)=0$. In this case, let $\widehat{\theta}_{q}$ be defined by $\delta_{b} \cdot \widehat{\theta}_{q}-q=\mu \cdot\left[g^{\prime}(0)-g^{\prime}\left(\bar{p}_{s_{q}, q}\right)\right]$. The proposition states that $s_{q}$ must be a debt security for $\theta>\widehat{\theta}_{q}$ but leaves $s_{q}(\theta) \in[0, \theta]$ undetermined for $\theta<\widehat{\theta}_{q}$. This difference stems from the boundedness of the marginal information cost $g^{\prime}$, which allows $m_{s_{q}, q}(\theta)=0$ for $\theta<\widehat{\theta}_{q}$ so that the security payoff does not matter. In contrast, unbounded $g^{\prime}$ disciplines the security payoff for all states. Intuitively, this setting nests the special case without information cost, in which the marginal information cost is zero, and thus leads to the largest region of indeterminacy $\left\{\theta<\delta_{b}^{-1} q\right\}$. By comparing the special case of zero information cost and those with bounded or unbounded marginal information costs, we see that i) the flexibility of choosing information acquisition strategy $m$ state by state guarantees the flat tail feature of the optimal securities, and ii) the pointwise marginal cost of information acquisition $g^{\prime}$ leads to the 45-degree line portion of optimal securities. These two features together lead to the optimality of debt.

\subsection{Multiple Underlying Assets}

My baseline model can be naturally generalized to accommodate multiple underlying assets. In this case, the fundamental state is a vector $\vec{\theta} \in \mathbb{R}_{+}^{N}$, with each component of $\vec{\theta}$ representing the cash flow of one underlying asset. The security payoff is contingent on $\vec{\theta}$, denoted by $s(\vec{\theta})$. Accordingly, the limited liability constraint becomes $s(\vec{\theta}) \in\left[0, \sum_{n=1}^{N} \theta_{n}\right]$. The rest of the setup remains the same as in the baseline model. I state the results in the following proposition.

used to exclude the case $\eta(\theta)<0$ because, when $\theta$ is small, the limited liability constraint $s_{q}(\theta) \leq \theta$ could be binding. 
Proposition 8 Let $\left(s^{*}, q^{*}\right)$ denote the seller's optimal offer and

$$
\bar{p}_{s^{*}, q^{*}}=\mathbb{E} m_{s^{*}, q^{*}}(\vec{\theta})
$$

be the corresponding probability of the trade taking place. Then i) trade occurs with positive probability, that is, $\bar{p}_{s^{*}, q^{*}}>0$; ii) there exists a $D^{*}>0$ such that the seller's optimal security takes the form

$$
s^{*}(\vec{\theta})=\min \left(\sum_{n=1}^{N} \theta_{n}, D^{*}\right),
$$

that is, a debt security backed by the sum of all the underlying cash flows.

It is optimal for the seller to pool all the assets and then issue a debt security backed by the pool. The intuition of the optimality of a debt security is analogous to that of the baseline model. The additional insight here is that the payoff of the optimal security depends only on the sum of the cash flows of the underlying assets. This is to deter the buyer from distinguishing between any two states $\left(\theta_{1}, \theta_{2}, \cdots, \theta_{N}\right)$ and $\left(\theta_{1}^{\prime}, \theta_{2}^{\prime}, \cdots, \theta_{N}^{\prime}\right)$ such that $\sum_{n=1}^{N} \theta_{n}=\sum_{n=1}^{N} \theta_{n}^{\prime}$. In other words, pooling discourages information acquisition by making the cash flow from each individual asset irrelevant, given their sum. In contrast, if the seller pools $N$ debts backed by each asset separately, for example, $s(\vec{\theta})=\sum_{n=1}^{N} \min \left(\theta_{n}, D_{n}\right)$, the buyer will acquire extra information about each asset. This simply reduces the surplus..

\subsection{Bertrand Competition between Buyers}

The baseline model focuses on the interaction between one seller and one buyer. A possible extension of the model is to consider multiple buyers acquiring information and bidding for the security. For instance, consider introducing two ex ante identical buyers in the baseline model. The seller first proposes the security to the buyers. Both buyers simultaneously acquire information flexibly and submit their bids to the seller. The buyer who submits the higher bid wins the security and pays his bid. This extension thus combines security design with a first-price common-value auction with flexible information acquisition.

In addition to the interaction between the seller and the buyers, a new strategic interaction between the buyers emerges. To illustrate this, consider two extreme scenarios. First, information is infinitely costly, that is, $\mu=\infty$. In this scenario, the two buyers cannot acquire information and bid according to their common prior belief. For any security $s$, it is clear that both buyers bid $\delta_{b} \mathbb{E} s(\theta)$, and the seller's optimal security is $s(\theta)=\theta$. In the second extreme scenario, information is costless, that is, $\mu=0$. In this scenario, the two buyers acquire complete information about $\theta$ and thus arrive at a common posterior belief. For any security $s$ and state $\theta$, it is clear that both buyers bid $\delta_{b} s(\theta)$ and the seller's optimal security is again $s(\theta)=\theta$. In both extreme scenarios, the gains from trade are maximized, and all surplus $\left(\delta_{b}-\delta_{s}\right) \mathbb{E} \theta$ goes to the seller. 
Now consider the intermediate scenario $\mu \in(0, \infty)$ and focus on symmetric equilibria. Since information is costly and the buyers acquire information privately, they will have imperfect and conditionally independent signals about the fundamental. Because this is a common-value auction, their signals are affiliated but heterogeneous, resulting in the winner's curse, which in turn pushes their bids below the corresponding conditional expectation of $s(\theta)$ given their posterior beliefs. The seller's revenue must therefore be less than $\delta_{b} \mathbb{E} s(\theta)$. In other words, endogenous asymmetric information between the buyers emerges. Importantly, as seen from the discussion about the two extreme scenarios above, information asymmetry between the buyers has a non-monotonic relationship with information acquisition. My conjecture is that whether it is optimal for the seller to encourage information acquisition depends on whether doing so helps reduce information asymmetry between the buyers. When information is cheap enough, it might be easier to encourage information acquisition to reduce the information asymmetry, so that a steep security seems optimal. In contrast, when information is very expensive, it might be easier to discourage information acquisition to reduce the information asymmetry, so that a flat security seems optimal.

In fact, the conjecture above resembles the well-known "linkage-principle" discussion in Milgrom and Weber (1982) that when bidders have no private information or know the true value of the item perfectly, they capture no information rent, and all the surplus goes to the seller. Thus, when information is infinitely costly or costless, the optimal security is always $s(\theta)=\theta$, and the gains from trade are maximized. However, when $\mu \in(0, \infty)$, the bidders' private signals become affiliated, and the seller's surplus is not monotone in $\mu$ because now bidders are worried about the winner's curse and may shade their bids. According to Milgrom and Weber (1982), whether more precise information for the bidders would lead to a higher seller surplus depends on many conditions even under the simplest common-value auction setting. To consider this specific problem poses an interesting theoretical question in the auction literature by itself, but to potentially incorporate it into a security design setting with flexible information acquisition is beyond the scope of this paper.

Additionally, since the strategy space for a buyer's bids will become continuous in this extension, a binary signal information structure is no longer optimal. This would require a complete overhaul of the current baseline model. This is technically challenging since, in the literature, there is no known solution to general continuous-choice decision problems with flexible information acquisition. ${ }^{19}$ A solution to the problem thus leads to an entirely different theoretical question.

Although this extension is interesting, for the reasons above, it requires a completely different modeling framework and machinery. Thus I leave this extension for future studies.

\footnotetext{
${ }^{19}$ To the best of my knowledge, the only paper exploring this is Jung, Kim, Matejka, and Sims (2019), which shows that a solution may exist only under rare mathematical conditions such as the objective function being able to be analytically extended to the complex plane.
} 


\section{Conclusion}

This paper studies security design through its interaction with information acquisition. To raise liquidity an impatient seller designs a security backed by her asset in place, and a buyer decides whether to purchase the security. The buyer naturally has incentives to acquire qualitatively different information when facing different securities, which in turn affects security design. I introduce the framework of flexible information acquisition to accommodate such interactions, in which issuing debt is shown to be uniquely optimal. The optimality of debt stems from an important feature of the model: fixed aggregate cash flow. Information acquisition creates an adverse selection problem that reduces social surplus. The seller thus designs the security to discourage information acquisition. The ideal security should have a constant security payoff, which gives the buyer no incentive to acquire information. When this constant payoff is too high to be supported by the underlying cash flow, the limited liability constraint binds and gives rise to the 45-degree-line portion of the debt security.

I also consider several alternative settings. I show that debt remains optimal if there are multiple underlying assets and if the information cost satisfies alternative assumptions. I show that debt is no longer optimal if the aggregate cash flow is variable, which indicates that a fixed aggregate cash flow is crucial to the result of the baseline model. I also consider Bertrand competitions with multiple buyers flexibly acquiring information and competitively bidding for the security. I argue that adding the competitive buyers changes the nature of the strategic interaction and conjecture that debt is not optimal. In the online appendix, I examine three additional variants of the baseline model: switching the roles of the buyer and seller, allowing for arbitrary allocation of bargaining power between the two parties, and letting the seller acquire information about the underlying asset in addition to designing the security.

Methodologically, this paper provides a new framework for incorporating flexible information acquisition into contract theory models. There are two major avenues left for future research. First, for simplicity, this paper allows only one party to have the flexibility in information acquisition. Multiple parties are naturally entitled to such flexibility in contract theory models, such as those involving multilateral or hierarchical contracting. For example, in the context of this paper, in addition to Bertrand competition between multiple buyers, the seller may take into account the fact that the buyer himself may have to subsequently finance his purchase from someone else, who is also entitled to the flexibility in acquiring information about the underlying asset but cannot directly transact with the seller. Incorporating multiple parties with such flexibility is interesting, but challenging.

Second, in my setting of information cost, no state is characterized by special difficulty in information acquisition. This homogeneity in information acquisition provides a fair

benchmark for the analysis but may not necessarily be realistic. For example, some extreme 
states may be much easier to identify than normal states. They may potentially attract more attention, and the optimal security should be designed accordingly in order to mitigate this effect. This limits the applicability of my baseline model in practice. It is straightforward to see that the optimal security can be manipulated by making information acquisition nonhomogeneous over the states. Hence, it makes sense to consider this variation in a more practical application where the heterogeneity of the states can be identified case by case.

\section{References}

Antic, N. (2015). Contracting with unknown technologies. Working paper, Princeton University.

Axelson, U. L. F. (2007). Security design with investor private information. Journal of Finance 62 (6), 2587-2632.

Biais, B. and T. Mariotti (2005). Strategic liquidity supply and security design. Review of Economic Studies 72(3), 615-649.

Blackwell, D. (1953). Equivalent comparisons of experiments. Annals of Mathematical Statistics $24(2), 265-272$.

Boot, A. W. A. and A. V. Thakor (1993). Security design. Journal of Finance 48(4), 1349-1378.

Dang, T. V., G. Gorton, and B. Holmström (2015). Ignorance, debt and financial crises. Unpublished working paper, Columbia University.

Dang, T. V., G. Gorton, and B. Hölmström (2015). Information sensitivity of a security. Unpublished working paper, Columbia University.

DeMarzo, P. M. (2005). The pooling and tranching of securities: A model of informed intermediation. Review of Financial Studies 18(1), 1-35.

DeMarzo, P. M. and D. Duffie (1999). A liquidity-based model of security design. Econometrica $67(1), 65-100$.

Farhi, E. and J. Tirole (2015). Liquid bundles. Journal of Economic Theory 158, 634-655.

Faure-Grimaud, A. (2000). Product market competition and optimal debt contracts: The limited liability effect revisited. European Economic Review 44 (10), 1823-1840.

Fulghieri, P. and D. Lukin (2001). Information production, dilution costs, and optimal security design. Journal of Financial Economics 61(1), 3-42.

Hennessy, C. (2013). A theory of abs design based on rational noise-traders. Working paper, London Business School. 
Holmstrom, B. and P. Milgrom (1987). Aggregation and linearity in the provision of intertemporal incentives. Econometrica 55(2), 303-328.

Hébert, B. $(2017,12)$. Moral Hazard and the Optimality of Debt. The Review of Economic Studies 85(4), 2214-2252.

Inderst, R. and H. M. Mueller (2006). Informed lending and security design. Journal of Finance 61(5), 2137-2162.

Jung, J., J.-H. Kim, F. Matejka, and C. Sims (2019). Discrete actions in informationconstrained problems. The Review of Economic Studies (accepted).

Leland, H. E. and D. H. Pyle (1977). Informational asymmetries, financial structure, and financial intermediation. Journal of Finance 32(2), 371-387.

Milgrom, P. R. and R. J. Weber (1982). A theory of auctions and competitive bidding. Econometrica 50(5), 1089-1122.

Nachman, D. C. and T. H. Noe (1994). Optimal design of securities under asymmetric information. Review of Financial Studies 7(1), 1-44.

Sims, C. A. (1998). Stickiness. Carnegie-Rochester Conference Series on Public Policy $49(1), 317-356$.

Townsend, R. M. (1979). Optimal contracts and competitive markets with costly state verification. Journal of Economic Theory 21(2), 265-293.

Woodford, M. (2008). Inattention as a source of randomized discrete adjustment. Working paper, Columbia University.

Yang, M. and Y. Zeng (2018, 08). Financing Entrepreneurial Production: Security Design with Flexible Information Acquisition. The Review of Financial Studies 32(3), 819863. 


\section{A Appendix: Further Discussion of Assumption A2}

Conditions i) and ii) are needed to make $c(\cdot)$ a well-defined information cost. In particular, Condition i) is necessary and sufficient to guarantee the non-negativeness of information $\operatorname{cost} c(\cdot)$. The sufficiency follows Jensen's inequality. To understand the necessity, suppose that $g$ is not strictly convex. This implies that there exist $x_{1}, x_{2}$, and $\lambda \in(0,1)$, such that $g\left(x_{\lambda}\right) \geq \lambda g\left(x_{1}\right)+(1-\lambda) g\left(x_{2}\right)$, where $x_{\lambda}=\lambda x_{1}+(1-\lambda) x_{2}$. Since the prior $P$ is absolutely continuous with respect to Lebesgue's measure, there exists an event $A \subset \Theta$ with probability measure $\lambda$ under the prior $P$. Consider two information structures

$$
m(\theta)= \begin{cases}x_{1} & \text { if } \theta \in A \\ x_{2} & \text { if } \theta \notin A\end{cases}
$$

and

$$
m_{\lambda}(\theta)=x_{\lambda} \text { for all } \theta \in \Theta .
$$

The information structure $m_{\lambda}$ is a garbling of $m$ in the sense of Blackwell's ordering and is completely uninformative. The associated information cost is $c(m)=0$. In contrast, the cost of information structure $m$ is

$$
\begin{aligned}
c(m) & =\mu \cdot[\mathbb{E} g(m(\theta))-g(\mathbb{E} m(\theta))] \\
& =\mu \cdot\left[\lambda g\left(x_{1}\right)+(1-\lambda) g\left(x_{2}\right)-g\left(x_{\lambda}\right)\right] \\
& \leq 0,
\end{aligned}
$$

which does not make sense since $m$ is strictly more informative than $m_{\lambda}$. This suggests that $g$ should be strictly convex.

To see the point of Condition ii), note that if I switch the recommendations of the two signal realizations (i.e., "taking action 0" upon observing signal 1 and "taking action 1" upon observing signal 0), the information content remains the same and so does the information cost, which requires $c(m)=c(1-m)$. In particular, consider two information structures $\pi_{1}(x=1 \mid \theta)=m(\theta)$ and $\pi_{2}(x=1 \mid \theta)=1-m(\theta)$. For a given fundamental state $\theta, \pi_{1}$ generates signal 1 with probability $m(\theta)$ and signal 0 with probability $1-m(\theta)$. If the buyer follows the signal recommendation, his conditional probability of accepting the offer is $m(\theta)$. Instead, if the buyer chooses information structure $\pi_{2}$ but accepts the offer while the signal is 0 and rejects the offer while the signal is 1 , his conditional probability of accepting the offer remains $m(\theta)$. Hence, the information content of information structures $\pi_{1}$ and $\pi_{2}$ is the same and so are the strategies that can be implemented under them. If the structures incur different information costs, I can simply choose the lower one for the cost of both. This justifies my assumption that $g$ is symmetric.

Condition iii) is a technical condition assumed for the sake of tractability. It guarantees 
that the direct effect mentioned in the perturbation argument that precedes Proposition 5 is non-negative, so that the first order variational approach is sufficient for my main results. This condition is not necessary and could be relaxed. I stick to this condition for the sake of tractability and transparency, since relaxing it does not deliver new insights but complicates the analysis.

Conditions i), ii), iii) and iv) guarantee that the $\operatorname{limit}_{z \rightarrow \infty} \frac{f^{\prime}(z+1)}{f^{\prime}(z)}$ in Condition v) is well defined. In addition, $K \geq 0$ since $f^{\prime}=\frac{1}{g^{\prime \prime}}>0$. It also follows that $K \leq 1$ because otherwise $\int_{0}^{\infty} f^{\prime}(z) d z$ diverges, contradicting the fact that $\lim _{x \rightarrow \infty} f(x)=1$. Condition $\mathrm{v}$ ) excludes the non-generic case $K=1$ in which I cannot obtain an analytically clear criterion for information acquisition. Numerical examples suggest that the paper's main qualitative results hold. These numerical examples are available upon request.

I outline the derivation of Proposition 2 to show how Conditions iv) and v) result in criteria (5), (6) and (7).

Proposition 2 classifies the solution according to whether it involves information acquisition, and Condition iv) of Assumption A2 helps dichotomize the cases with and without triggering information acquisition. For states such that $\Delta u(\theta)>0$, the buyer should have accepted the offer but mistakenly rejects it with probability $1-m(\theta)$, because the marginal cost of avoiding this Type I error is $\lim _{m(\theta) \rightarrow 1} \mu \cdot\left[g^{\prime}(m(\theta))-g^{\prime}\left(p_{1}\right)\right]=\infty$, providing that $p_{1} \in(0,1)$. Similarly, for states such that $\Delta u(\theta)<0$, since $g(m)=g(1-m)$, the marginal cost of avoiding the Type II error is also infinity. Hence, if the buyer acquires information so that the unconditional probability of acceptance is $p_{1} \in(0,1)$, Condition iv) guarantees an interior solution to the information acquisition problem, that is, $m(\theta) \in(0,1)$ for all $\theta \in \mathbb{R}_{+}$. In contrast, without information acquisition, the buyer either always accepts or always rejects the offer, that is, either $m(\theta)=1$ for all $\theta \in \mathbb{R}_{+}$or $m(\theta)=0$ for all $\theta \in \mathbb{R}_{+}$.

Together with Condition iv), Condition v) allows me to obtain analytically clear criteria with which to determine whether it is worth acquiring information. These criteria-(5), (6) and (7)-are obtained by comparing the marginal value of information versus its marginal cost. To appreciate the intuition, I further use Equation (8) to express $m$ as a function of $\Delta u(\theta)$ parameterized by $p \in(0,1)$,

$$
m(\theta, p)=f\left(\mu^{-1} \Delta u(\theta)+g^{\prime}(p)\right)
$$

According to c) of Proposition 2, once the buyer decides to acquire information, his optimal strategy belongs to the parameterized family above. It is straightforward to verify that the parameterized strategy $m(\cdot, p)$ is state-contingent and thus involves information acquisition if $p \in(0,1)$. By allowing parameter $p$ to take values 1 and 0 , this family also encompasses strategies involving no information acquisition. To see this, note that when $p=1$, the parameterized strategy becomes $m(\theta, 1)=f\left(\mu^{-1} \Delta u(\theta)+\infty\right)=1$, which means accepting 
the offer with (unconditional) probability $p=1$. Similarly, when $p=0$, the parameterized strategy becomes $m(\theta, 0)=f\left(\mu^{-1} \Delta u(\theta)-\infty\right)=0$. Hence, in searching for the optimal strategy, it is sufficient to focus on the (one-dimension) parameterized family (17) with $p \in[0,1]$.

Let $J(p)=\mathbb{E} m(\theta, p)$ be the unconditional probability of acceptance associated with the parameterized strategy $m(\cdot, p)$. By definition, the optimal strategy $m\left(\cdot, p_{1}\right)$ should satisfy $J\left(p_{1}\right)=p_{1}$. Since $J(0)=0$ and $J(1)=1$, the two non-information-acquisition strategies $m(\cdot, 0)$ and $m(\cdot, 1)$ are natural candidates for the optimal strategy. To determine whether information acquisition is optimal, I consider whether the buyer obtains greater net benefit $V^{*}(m(\cdot, p))$ (i.e., gains net of information cost) by deviating from the extreme cases of $p=1$ and $p=0$. In particular, the net benefit of choosing $m(\cdot, p)$ is

$$
V^{*}(m(\cdot, p))=\mathbb{E}[\Delta u(\theta) \cdot m(\theta, p)]-\mu \cdot c(m(\cdot, p))
$$

and the marginal net benefit from increasing $p$ is

$$
\begin{aligned}
& \frac{d V^{*}(m(\cdot, p))}{d p} \\
= & \mathbb{E}\left(\left[\Delta u(\theta)-\mu \cdot g^{\prime}(m(\theta, p))+\mu \cdot g^{\prime}(J(p))\right] \cdot \frac{\partial m(\theta, p)}{\partial p}\right) \\
= & \mu \cdot\left[g^{\prime}(J(p))-g^{\prime}(p)\right] \cdot \mathbb{E} \frac{\partial m(\theta, p)}{\partial p},
\end{aligned}
$$

where the second equality follows (17). Since both $g^{\prime}$ and $f$ are strictly increasing, $\mathbb{E} \frac{\partial m(\theta, p)}{\partial p}>$ 0 , and thus

$$
\frac{d V^{*}(m(\cdot, p))}{d p}>0 \text { if and only if } J(p)>p .
$$

Since $J(1)=1$ and $J(0)=0$, the buyer benefits from acquiring information rather than always accepting (rejecting) the offer if and only if $J^{\prime}(1)>1\left(J^{\prime}(0)>1\right)$. To characterize $J^{\prime}(1)$ and $J^{\prime}(0)$, consider a function $t: \mathbb{R} \rightarrow \mathbb{R}$ such that $t(x)=\lim _{z \rightarrow \infty} \frac{f^{\prime}(x+z)}{f^{\prime}(z)}$. Since $t(x+y)=\lim _{z \rightarrow \infty} \frac{f^{\prime}(x+y+z)}{f^{\prime}(y+z)} \frac{f^{\prime}(y+z)}{f^{\prime}(z)}=t(x) \cdot t(y), t(\cdot)$ is an exponential function $t(x)=K^{x}$, where $K=t(1)=\lim _{z \rightarrow \infty} \frac{f^{\prime}(z+1)}{f^{\prime}(z)}$. Note that

$$
\frac{\partial m(\theta, p)}{\partial p}=f^{\prime}\left(\mu^{-1} \Delta u(\theta)+g^{\prime}(p)\right) g^{\prime \prime}(p)=\frac{f^{\prime}\left(\mu^{-1} \Delta u(\theta)+g^{\prime}(p)\right)}{f^{\prime}\left(g^{\prime}(p)\right)} .
$$

It immediately follows that $\lim _{p \rightarrow 1} \frac{\partial m(\theta, p)}{\partial p}=K^{\mu^{-1} \Delta u(\theta)}$ and

$$
J^{\prime}(1)=\mathbb{E}\left(\left.\frac{\partial m(\theta, p)}{\partial p}\right|_{p=1}\right)=\mathbb{E} K^{\mu^{-1} \Delta u(\theta)} .
$$


Hence, $\mathbb{E} K^{\mu^{-1} \Delta u(\theta)}>1$ implies $\frac{d V^{*}(m(\cdot, p))}{d p}<0$ for $p$ close to 1 , which means that the marginal value of information exceeds its marginal cost so that the buyer benefits from acquiring information rather than always accepting the offer. A symmetric argument applies to the case $\mathbb{E} K^{-\mu^{-1} \Delta u(\theta)}>1$, in which the buyer prefers acquiring information to always rejecting the offer. Therefore, once Inequality (7) holds, the optimal strategy involves information acquisition and is characterized by Equation (8). Similar analysis applies to Cases a) and b) and the complete proof is in the appendix.

It is worth noting that $K=0$ implies

$$
\lim _{p \rightarrow 1} \frac{\partial m(\theta, p)}{\partial p}=\left\{\begin{array}{c}
0 \text { if } \Delta u(\theta)>0 \\
1 \text { if } \Delta u(\theta)=0 \\
\infty \text { if } \Delta u(\theta)<0
\end{array}\right.
$$

and

$$
\lim _{p \rightarrow 0} \frac{\partial m(\theta, p)}{\partial p}=\left\{\begin{array}{c}
\infty \text { if } \Delta u(\theta)>0 \\
1 \text { if } \Delta u(\theta)=0 \\
0 \text { if } \Delta u(\theta)<0
\end{array},\right.
$$

which greatly simplify Criteria (5), (6) and (7). In particular, the buyer accepts (rejects) the offer without information acquisition if and only if $\Delta u(\theta) \geq 0(\Delta u(\theta) \leq 0)$ almost surely for all states. Once $\operatorname{Pr}(\Delta u(\theta)>0)>0$ and $\operatorname{Pr}(\Delta u(\theta)<0)>0$, the optimal strategy involves information acquisition and is characterized by Equation (8).

As shown by the above derivation, Condition iv) allows me to highlight the distinction between the case involving information acquisition and cases without information acquisition. In addition, Condition v) allows an analytical expression of the criteria by excluding the non-generic case $K=1$. When $K=1$, it is always true that $\mathbb{E} K^{\mu^{-1} \Delta u(\theta)}=1$ and $\mathbb{E} K^{-\mu^{-1} \Delta u(\theta)}=1$ whatever the payoff gain $\Delta u(\theta)$ is. Criteria (5), (6) and (7) thus lose predictive power. Additional conditions involving higher order derivatives of $f$ may help in the non-generic case $K=1$ but are too technical to convey interesting economics. I do not include the additional conditions in the paper.

Condition iv) assumes that the marginal cost of avoiding Type I and Type II errors is infinity, and Condition v) helps discipline the limiting behavior of this marginal cost. Many widely used measures of information cost, such as entropy reduction, possess this infinite-marginal-cost property, which is maintained throughout my baseline model of security design. It helps relate my general information costs to the rational inattention literature in which information costs take the form of entropy reduction. Alternatively, I analyze the case with bounded marginal costs in Subsection 5.2 and show that debt remains optimal whenever trade occurs. 


\section{B Mathematical Appendix}

Proof of Lemma 1.

Proof. Let $a(x)$ be the buyer's optimal decision rule upon observing $x \in X$, then

$$
\begin{aligned}
X_{1} & =\{x \in X: a(x)=1\}, \\
X_{0} & =\{x \in X: a(x)=0\}, \\
\text { and } X_{\text {indifferent }} & =\{x \in X: a(x) \in(0,1)\}
\end{aligned}
$$

form a partition of $X$. Construct a new information structure $(\widetilde{\pi}, \widetilde{X})$ with decision rule $\widetilde{a}(x)$ such that $\widetilde{X}=\left\{x_{0}, x_{1}\right\}$,

$$
\begin{aligned}
& \tilde{\pi}\left(x_{0} \mid \theta\right)=\int_{X_{0} \cup X_{\text {indifferent }}} \pi(x \mid \theta) d x, \\
& \tilde{\pi}\left(x_{1} \mid \theta\right)=\int_{X_{1}} \pi(x \mid \theta) d x,
\end{aligned}
$$

and

$$
\begin{aligned}
& \widetilde{a}\left(x_{0}\right)=0, \\
& \widetilde{a}\left(x_{1}\right)=1 .
\end{aligned}
$$

It is straightforward to see that the buyer enjoys the same expected payoff (excluding the information cost) under the two information structures. But information structure $\tilde{\pi}$ is a strict garbling of $\pi$ if $X_{\text {indifferent }}$ is non-empty or $\# X>2$. Then according to Assumption A1, information structure $\widetilde{\pi}$ is strictly cheaper than $\pi$. Therefore, the buyer will be strictly better off with $(\tilde{\pi}, \widetilde{X})$ and $\widetilde{a}$ if $X_{\text {indifferent }}$ is non-empty or $\# X>2$. This concludes the proof.

Proof of Proposition 2.

Proof. Suppose $m$ is an optimal strategy. Let $\varepsilon$ be any feasible perturbation function. The payoff from the perturbed strategy $m+\alpha \cdot \varepsilon$ is

$$
\begin{aligned}
& V^{*}(m+\alpha \cdot \varepsilon) \\
= & \mathbb{E}[(m(\theta)+\alpha \cdot \varepsilon(\theta)) \cdot \Delta u(\theta)] \\
& -\mu \cdot[\mathbb{E} g(m(\theta)+\alpha \cdot \varepsilon(\theta))-g(\mathbb{E}[m(\theta)+\alpha \cdot \varepsilon(\theta)])],
\end{aligned}
$$

where the expectation operator $\mathbb{E}(\cdot)$ is with respect to $\theta$ under prior $P$. A perturbation $\varepsilon$ is feasible with respect to $m$ if $\exists \alpha>0$, s.t. $\forall \theta \in \Theta, m(\theta)+\alpha \cdot \varepsilon(\theta) \in[0,1]$. Then the first 
order variation is

$$
\left.\frac{d V^{*}(m+\alpha \cdot \varepsilon)}{d \alpha}\right|_{\alpha=0}=\mathbb{E}\left(\varepsilon(\theta) \cdot\left[\Delta u(\theta)-\mu \cdot\left(g^{\prime}(m(\theta))-g^{\prime}\left(p_{1}\right)\right)\right]\right) .
$$

Note that

$$
\Delta u(\theta)-\mu \cdot\left(g^{\prime}(m(\theta))-g^{\prime}\left(p_{1}\right)\right)
$$

is the Fréchet derivative of $V^{*}(\cdot)$ at $m$. Hence the tangent hyperplane at $m$ can be expressed as

$\left\{(\widetilde{m}, v) \in M \times \mathbb{R}: v-V^{*}(m)=\mathbb{E}\left(\left[\Delta u(\theta)-\mu g^{\prime}(m(\theta))+\mu g^{\prime}(\mathbb{E} m(\theta))\right](\widetilde{m}(\theta)-m(\theta))\right)\right\}$.

Since the information cost $c(\cdot)$ respects Blackwell's ordering, it is strictly convex and hence $V^{*}(\cdot)$ is a strictly concave functional on $M$. This implies i) the optimal strategy exists and is unique, and ii) $V^{*}$ is upper bounded by the hyperplane tangent at any $m \in M$, i.e., $\forall m, \widetilde{m} \in M$,

$$
\begin{aligned}
& V^{*}(\widetilde{m})-V^{*}(m) \\
\leq & \mathbb{E}\left(\left[\Delta u(\theta)-\mu \cdot g^{\prime}(m(\theta))+\mu \cdot g^{\prime}\left(\int_{\Theta} m(\theta) d P(\theta)\right)\right](\widetilde{m}(\theta)-m(\theta))\right) .
\end{aligned}
$$

This inequality is strict when

$$
m \in M^{o} \triangleq M \backslash\{m \in M: m(\theta) \text { is a constant a.s. }\} .
$$

This observation is helpful later in my proof.

The optimality of $m$ requires $\left.\frac{d V^{*}(m+\alpha \cdot \varepsilon)}{d \alpha}\right|_{\alpha=0} \leq 0$ for all feasible perturbation $\varepsilon$. Hence it must be true that

$$
\Delta u(\theta)-\mu \cdot\left(g^{\prime}(m(\theta))-g^{\prime}\left(p_{1}\right)\right)\left\{\begin{array}{lc}
\geq 0 & \text { if } m(\theta)=1 \\
=0 & \text { if } m(\theta) \in(0,1) \\
\leq 0 & \text { if } m(\theta)=0
\end{array} .\right.
$$

Note that $\operatorname{Pr}(m(\theta)=1)>0$ implies $\operatorname{Pr}(m(\theta)=1)=1$. Otherwise,

$$
p_{1}=\mathbb{E} m(\theta)<1
$$

implies

$$
\Delta u(\theta)-\mu \cdot\left(g^{\prime}(m(\theta))-g^{\prime}\left(p_{1}\right)\right)=-\infty
$$

for all $\theta$ in set

$$
B=\{\theta \in \Theta: m(\theta)=1\} .
$$


Thus $\varepsilon(\theta)=-1_{B}$ is a feasible perturbation and

$$
\begin{aligned}
& \left.\frac{d V^{*}(m+\alpha \cdot \varepsilon)}{d \alpha}\right|_{\alpha=0} \\
= & \int_{B}(-\infty) \cdot(-1) d P(\theta) \\
= & +\infty,
\end{aligned}
$$

which contradicts the optimality of $m$. Hence, $\operatorname{Pr}(m(\theta)=1)>0$ if and only if $\operatorname{Pr}(m(\theta)=1)=$ 1. The same argument suggests that $\operatorname{Pr}(m(\theta)=0)>0$ if and only if $\operatorname{Pr}(m(\theta)=0)=1$. Therefore, the optimal strategy $m$ must be one of the three scenarios: a) $p_{1}=0$, i.e., $m(\theta)=0$ a.s.; b) $p_{1}=1$, i.e., $m(\theta)=1$ a.s.; c) $p_{1} \in(0,1)$ and $m(\theta) \in(0,1)$ a.s..

I first search for the sufficient condition for scenario c). According to $(18), m(\theta) \in(0,1)$ a.s. implies

$$
\Delta u(\theta)-\mu \cdot\left(g^{\prime}(m(\theta))-g^{\prime}\left(p_{1}\right)\right)=0 \text { a.s. } .
$$

Thus (19) implies

$$
m(\theta)=f\left(\mu^{-1} \Delta u(\theta)+g^{\prime}\left(p_{1}\right)\right),
$$

where $f$ is the inverse of $g^{\prime}$. Let

$$
M_{1}=\left\{m(\theta, p)=f\left(\mu^{-1} \Delta u(\theta)+g^{\prime}(p)\right): p \in[0,1]\right\}
$$

and

$$
J(p)=\mathbb{E} m(\theta, p),
$$

then there exists $p_{1} \in[0,1]$ such that $m\left(\cdot, p_{1}\right) \in M_{1}$ is an optimal strategy. Note that $J\left(p_{1}\right)=p_{1}$ is a necessary condition for the optimality of $m\left(\cdot, p_{1}\right)$.

Since $m\left(\cdot, p_{1}\right) \in M_{1} \subset M$, the original problem is reduced to

$$
\max _{p \in[0,1]} V^{*}(m(\cdot, p))=\mathbb{E}[\Delta u(\theta) \cdot m(\theta, p)]-\mu \cdot c(m(\cdot, p)) .
$$

The first order derivative with respect to $p$ is

$$
\begin{aligned}
& \frac{d V^{*}(m(\cdot, p))}{d p} \\
= & \mathbb{E}\left(\left[\Delta u(\theta)-\mu \cdot g^{\prime}(m(\theta, p))+\mu \cdot g^{\prime}(J(p))\right] \cdot \frac{\partial m(\theta, p)}{\partial p}\right) .
\end{aligned}
$$

By definition,

$$
\Delta u(\theta)-\mu \cdot g^{\prime}(m(\theta, p))=-\mu \cdot g^{\prime}(p)
$$


thus

$$
\frac{d V^{*}(m(\cdot, p))}{d p}=\mu \cdot\left[g^{\prime}(J(p))-g^{\prime}(p)\right] \cdot \mathbb{E} \frac{\partial m(\theta, p)}{\partial p} .
$$

Since

$$
\frac{\partial m(\theta, p)}{\partial p}>0
$$

for all $\theta \in \Theta$,

$$
\frac{d V^{*}(m(\cdot, p))}{d p} \geq 0
$$

if and only if

$$
g^{\prime}(J(p))-g^{\prime}(p) \geq 0 .
$$

Since $g^{\prime}$ is strictly increasing, I obtain that

$$
\frac{d V^{*}(m(\cdot, p))}{d p} \geq 0
$$

if and only if

$$
J(p) \geq p .
$$

In order to be a global maximum, $m\left(\cdot, p_{1}\right)$ must first be a local maximum within $M_{1}$. This requires

$$
J\left(p_{1}\right)=p_{1} .
$$

But (21) is not sufficient. The sufficient condition for $m\left(\cdot, p_{1}\right)$ to be a local maximum within $M_{1}$ is

$$
\exists \text { neighborhood }\left(p_{1}-\beta, p_{1}+\beta\right)
$$

$$
\begin{aligned}
& \text { s.t. } J(p) \geq p \text { for all } p \in\left(p_{1}-\beta, p_{1}\right] \\
& \text { and } J(p) \leq p \text { for all } p \in\left[p_{1}, p_{1}+\beta\right) .
\end{aligned}
$$

To conduct the local maximum analysis, I introduce a function $t(x)=\lim _{z \rightarrow \infty} \frac{f^{\prime}(x+z)}{f^{\prime}(z)}$. Since $g^{\prime}$ is continuously differentiable, $t(x)$ exists for all $x \in \mathbb{R}$. By definition,

$$
\begin{aligned}
t(x+y) & =\lim _{z \rightarrow \infty} \frac{f^{\prime}(x+y+z)}{f^{\prime}(z)}=\lim _{z \rightarrow \infty} \frac{f^{\prime}(x+y+z)}{f^{\prime}(y+z)} \frac{f^{\prime}(y+z)}{f^{\prime}(z)} \\
& =\lim _{z \rightarrow \infty} \frac{f^{\prime}(x+y+z)}{f^{\prime}(y+z)} \lim _{z \rightarrow \infty} \frac{f^{\prime}(y+z)}{f^{\prime}(z)}=t(x) \cdot t(y) .
\end{aligned}
$$

This implies that $t(x)$ is an exponential function taking the form $t(x)=K^{x}$, where $K=$ $t(1)$. It is clear that $K \geq 0$ because $g^{\prime \prime}>0$. By definition, it must be true that $K<1$. Otherwise $f^{\prime}(z)$ decreases too slow to make $\int_{-\infty}^{\infty} f^{\prime}(z) d z$ converge, contradicting the fact 
that $\lim _{x \rightarrow \infty} f(x)=1$. Note that

$$
\begin{gathered}
J(0)=0, J(1)=1, \\
\left.\frac{d J}{d p}\right|_{p=0}=\mathbb{E}\left(\lim _{z \rightarrow \infty} \frac{f^{\prime}\left(\mu^{-1} \Delta u(\theta)-z\right)}{f^{\prime}(-z)}\right)=\mathbb{E} K^{-\mu^{-1} \Delta u(\theta)}
\end{gathered}
$$

and

$$
\left.\frac{d J}{d p}\right|_{p=1}=\mathbb{E}\left(\lim _{z \rightarrow \infty} \frac{f^{\prime}\left(\mu^{-1} \Delta u(\theta)+z\right)}{f^{\prime}(z)}\right)=\mathbb{E} K^{\mu^{-1} \Delta u(\theta) .}
$$

I proceed by discussing four possible cases.

\section{Case i):}

$$
\mathbb{E} K^{-\mu^{-1} \Delta u(\theta)}>1
$$

and

$$
\mathbb{E} K^{\mu^{-1} \Delta u(\theta)}>1
$$

In this case, $J(p)>p$ for $p$ close enough to 0 and $J(p)<p$ for $p$ close enough to 1. Since $J(p)$ is continuous, the set $\{p \in(0,1): J(p)=p\}$ is non-empty. For any $p_{1} \in$ $\{p \in(0,1): J(p)=p\}$, the Fréchet derivative at $m\left(\cdot, p_{1}\right)$ is

$$
\Delta u(\theta)-\mu \cdot g^{\prime}\left(m\left(\theta, p_{1}\right)\right)+\mu \cdot g^{\prime}\left(J\left(p_{1}\right)\right)=0
$$

and thus $m\left(\cdot, p_{1}\right)$ is a critical point of functional $V^{*}(\cdot)$. Since $m\left(\cdot, p_{1}\right) \in M^{o}$, the observation mentioned above implies

$$
V^{*}(\widetilde{m})-V^{*}\left(m\left(\cdot, p_{1}\right)\right)<0
$$

for all $\widetilde{m} \neq m$. Hence, $V^{*}\left(m\left(\cdot, p_{1}\right)\right)$ is strictly higher than the values achieved at any other $\widetilde{m} \in M$, i.e.,

$$
\{p \in(0,1): J(p)=p\}=\left\{p_{1}\right\}
$$

and $m\left(\cdot, p_{1}\right)$ is the unique global maximum. This proves $(7)$.

\section{Case ii):}

$$
\mathbb{E} K^{-\mu^{-1} \Delta u(\theta)}>1
$$

and

$$
\mathbb{E} K^{\mu^{-1} \Delta u(\theta)} \leq 1
$$

In this case, $J(p)>p$ for $p$ close enough to 0 and $J(p) \geq p$ for $p$ close enough to 1 . I show that $J(p)>p$ for all $p \in(0,1)$. If this is not true, let $p_{1}=\sup \{p \in(0,1): J(p) \leq p\}<1$ and thus $m\left(\cdot, p_{1}\right) \in M^{o}$. On the one hand, the continuity of $J(p)$ implies $J\left(p_{1}\right)=p_{1}$. Hence $m\left(\cdot, p_{1}\right)$ is a critical point of functional $V^{*}(\cdot)$. By the same argument as in Case i), I know that $m\left(\cdot, p_{1}\right)$ is the unique global maximum. On the other hand, by the construction 
of $p_{1}, J(p)>p$ for all $p \in\left(p_{1}, 1\right)$. Then $V^{*}(m(\cdot, p))>V^{*}\left(m\left(\cdot, p_{1}\right)\right)$ for all $p \in\left(p_{1}, 1\right)$ which contradicts the unique optimality of $m\left(\cdot, p_{1}\right)$. Therefore, $J(p)>p$ for all $p \in(0,1)$ and the optimal strategy cannot be an interior point of $M_{1}$ (i.e., it cannot be the case $p_{1} \in(0,1)$.) Then according to my previous discussion, only scenarios a) that $p_{1}=0$ and scenario b) that $p_{1}=1$ are possible. Since $J(p)>p$ for all $p \in(0,1)$, I obtain that

$$
V^{*}(m(\cdot, 1))>V^{*}(m(\cdot, 0)) .
$$

Hence, $p_{1}=1$, i.e., $m(\theta)=1$ a.s. is the unique optimal strategy. Note that (22) is a direct implication of (23) according to Jensen's inequality. Thus this case can be summarized by (23) alone. This proves (6).

\section{case iii):}

$$
\mathbb{E} K^{-\mu^{-1} \Delta u(\theta)} \leq 1
$$

and

$$
\mathbb{E} K^{\mu^{-1} \Delta u(\theta)}>1
$$

Note that this case can be summarized by (24) alone, since (25) is a direct implication of (24) according to Jensen's inequality. In this case, by the same argument as in case ii), $m(\theta)=0$ a.s. is the unique optimal strategy. This proves (5).

case iv):

$$
\mathbb{E} K^{-\mu^{-1} \Delta u(\theta)} \leq 1
$$

and

$$
\mathbb{E} K^{\mu^{-1} \Delta u(\theta)} \leq 1
$$

Case iv) cannot exists since Jensen's inequality implies

$$
\mathbb{E} K^{\mu^{-1} \Delta u(\theta)}=\mathbb{E}\left[\frac{1}{K^{-\mu^{-1} \Delta u(\theta)}}\right] \geq\left[\mathbb{E} K^{-\mu^{-1} \Delta u(\theta)}\right]^{-1},
$$

which suggests (26) and (27) hold with equality. This is true only if $\Delta u(\theta)=0$ almost surely, a trivial case excluded by my assumption.

Since cases i), ii) and iii) exhaust all possibilities, for each case, the corresponding conditions are not only sufficient but also necessary.

\section{Proof of Proposition 3.}

Proof. I prove by constructing a debt security that generates positive expected payoff to the seller. Let $\beta \in\left(\delta_{s} \delta_{b}^{-1}, 1\right)$ and

$$
y(q)=\mathbb{E} \min \left(\theta, \beta \delta_{s}^{-1} q\right) .
$$


Since $P$ is a continuous distribution and $\beta^{-1} \delta_{s} \delta_{b}^{-1}<1$, there exists $q_{0}>0$ s.t.

$$
\operatorname{Pr}\left(\theta \geq \beta \delta_{s}^{-1} q\right)>\beta^{-1} \delta_{s} \delta_{b}^{-1}
$$

for all $q \in\left[0, q_{0}\right]$. Hence for any $q \in\left(0, q_{0}\right)$,

$$
\begin{aligned}
y^{\prime}(q) & =\operatorname{Pr}\left(\theta \geq \beta \delta_{s}^{-1} q\right) \cdot \beta \delta_{s}^{-1} \\
& >\beta^{-1} \delta_{s} \delta_{b}^{-1} \cdot \beta \delta_{s}^{-1}=\delta_{b}^{-1} .
\end{aligned}
$$

Note that

$$
y(0)=0
$$

which implies that

$$
y(q)>\delta_{b}^{-1} q
$$

for all $q \in\left(0, q_{0}\right)$.

Consider a debt

$$
s(\theta)=\min (\theta, D)
$$

with face value $D=\beta \delta_{s}^{-1} q$ and price $q \in\left(0, q_{0}\right)$. The buyer's payoff gain from accepting this offer over rejecting it is

$$
\Delta u(\theta)=\delta_{b} \cdot s(\theta)-q .
$$

By Jensen's inequality, I obtain

$$
\begin{aligned}
& \mathbb{E} K^{-\mu^{-1} \Delta u(\theta)} \\
\geq & K^{-\mu^{-1} \mathbb{E} \Delta u(\theta)} \\
= & K^{-\mu^{-1}\left[\delta_{b} \cdot y(q)-q\right]} \\
> & 1,
\end{aligned}
$$

which implies $\bar{p}_{s, q}>0$ according to Proposition 2. Then, the seller's expected payoff from this offer is

$$
\begin{aligned}
W(s, q) & =\mathbb{E}\left(m_{s, q}(\theta) \cdot\left[q-\delta_{s} \cdot s(\theta)\right]\right) \\
& \geq \mathbb{E}\left(m_{s, q}(\theta) \cdot\left[q-\delta_{s} \cdot \beta \delta_{s}^{-1} q\right]\right) \\
& =(1-\beta) q \cdot \bar{p}_{s, q}>0 .
\end{aligned}
$$

By proposing the optimal offer $\left(s^{*}, q^{*}\right)$, the seller's expected payoff should be no less than $W(s, q)>0$. This directly implies $\bar{p}_{s^{*}, q^{*}}>0$, since $\bar{p}_{s^{*}, q^{*}}=0$ always generates zero expected payoff to the seller.

\section{Proof of Proposition 4.}


Proof. For any given $q>0$, let $s_{q}$ denote the optimal security that does not induce information acquisition. The seller's problem is

$$
\min _{s} \delta_{s} \cdot \mathbb{E} s(\theta)
$$

such that

$$
q \leq-\kappa^{-1} \mu \ln \mathbb{E} \exp \left(-\kappa \mu^{-1} \delta_{b} \cdot s(\theta)\right)
$$

and

$$
s(\theta) \in[0, \theta] .
$$

I first consider the Lagrangian without the limited liability constraint, which is

$$
J=\delta_{s} \cdot \mathbb{E} s(\theta)+\lambda \cdot\left[\kappa^{-1} \mu \ln \mathbb{E} \exp \left(-\kappa \mu^{-1} \delta_{b} \cdot s(\theta)\right)+q\right]
$$

where $\lambda$ is the Lagrangian multiplier. The first order derivative with respect to $s(\theta)$ is

$$
\begin{aligned}
& \delta_{s}-\lambda \delta_{b}\left[\mathbb{E} \exp \left(-\kappa \mu^{-1} \delta_{b} \cdot s(\theta)\right)\right]^{-1} \exp \left(-\kappa \mu^{-1} \delta_{b} \cdot s(\theta)\right) \\
= & \delta_{s}-\lambda \delta_{b} \exp \left(\kappa \mu^{-1}\left[q-\delta_{b} \cdot s(\theta)\right]\right) \triangleq r(\theta \mid s) .
\end{aligned}
$$

Let

$$
\begin{gathered}
A_{0}=\left\{\theta \in \Theta: \theta>0, s_{q}(\theta)=0\right\}, \\
A_{1}=\left\{\theta \in \Theta: \theta>0, s_{q}(\theta) \in(0, \theta)\right\}
\end{gathered}
$$

and

$$
A_{2}=\left\{\theta \in \Theta: \theta>0, s_{q}(\theta)=\theta\right\} .
$$

Then $\left\{A_{0}, A_{1}, A_{2}\right\}$ is a partition of $\Theta \backslash\{0\}$. Now consider the limited liability constraint, which together with the optimality of $s_{q}$ implies

$$
r\left(\theta \mid s_{q}\right)\left\{\begin{array}{ll}
\geq 0 & \text { if } \theta \in A_{0} \\
=0 & \text { if } \theta \in A_{1} \\
\leq 0 & \text { if } \theta \in A_{2}
\end{array} .\right.
$$

Note that my argument for (10) implies $\lambda>0$. Hence, once there exists a $\theta^{\prime} \in A_{0}$, I obtain $r\left(\theta^{\prime} \mid s_{q}\right) \geq 0$ which together with (10) further implies $r\left(\theta^{\prime \prime} \mid s_{q}\right)>0$ for all $\theta^{\prime \prime} \in A_{1} \cup A_{2}$, which is a contradiction. Therefore,

$$
\operatorname{Pr}\left(A_{0}\right)=0 .
$$


For any $\theta^{\prime} \in A_{1},(32)$ implies $r\left(\theta^{\prime} \mid s_{q}\right)=0$, i.e.,

$$
\delta_{s}=\lambda \delta_{b} \exp \left(\kappa \mu^{-1}\left[q-\delta_{b} \cdot s_{q}\left(\theta^{\prime}\right)\right]\right)
$$

Hence,

$$
s_{q}\left(\theta^{\prime}\right)=\kappa^{-1} \mu \delta_{b}^{-1} \cdot\left[\ln \left(\lambda \delta_{b}\right)-\ln \delta_{s}\right]+\delta_{b}^{-1} q
$$

is a constant for all $\theta^{\prime} \in A_{1}$. Let $D(q)$ denote this constant.

For any $\theta^{\prime} \in A_{2}$, (32) implies $r\left(\theta^{\prime}\right) \leq 0$, so that $\theta^{\prime}=s_{q}\left(\theta^{\prime}\right) \leq D(q)$. Then, it immediately follows that

$$
s_{q}(\theta)=\min (\theta, D(q)) .
$$

Finally, I characterize the optimal level of the price and the face value. By replacing $q$ with $-\kappa^{-1} \mu \ln \mathbb{E} \exp \left(-\kappa \mu^{-1} \delta_{b} \cdot s(\theta)\right)$, I obtain the seller's objective

$$
\min _{s} \kappa^{-1} \mu \ln \mathbb{E} \exp \left(-\kappa \mu^{-1} \delta_{b} \cdot s(\theta)\right)+\delta_{s} \cdot \mathbb{E} s(\theta)
$$

such that

$$
s(\theta) \in[0, \theta] .
$$

As argued before, the first order derivative with respect to $s(\theta)$ equals zero for $s(\theta) \in(0, \theta)$.

This implies

$$
s(\theta)=\kappa^{-1} \mu \delta_{b}^{-1} \cdot\left[\ln \delta_{b}-\ln \delta_{s}\right]+\delta_{b}^{-1} q \triangleq D^{*}(q)
$$

and $s(\theta)=\min \left(\theta, D^{*}(q)\right)$. Let

$$
h(q)=-\kappa^{-1} \mu \ln \mathbb{E} \exp \left(-\kappa \mu^{-1} \delta_{b} \cdot \min \left(\theta, D^{*}(q)\right)\right) .
$$

I show that the optimal price $q^{*}>0$ and it is the unique fixed point of $h(q)$.

By (10), I obtain

$$
\begin{aligned}
q^{*} & =-\kappa^{-1} \mu \ln \mathbb{E} \exp \left(-\kappa \mu^{-1} \delta_{b} \cdot \min \left(\theta, \kappa^{-1} \mu \delta_{b}^{-1} \cdot\left[\ln \delta_{b}-\ln \delta_{s}\right]+\delta_{b}^{-1} q^{*}\right)\right) \\
& =h\left(q^{*}\right)
\end{aligned}
$$

Hence $q^{*}$ is a fixed point of $h(q)$.

It is obvious that $h(0)>0$. Also note that

$$
\begin{aligned}
& h^{\prime}(q) \\
= & {\left[\mathbb{E} \exp \left(-\kappa \mu^{-1} \delta_{b} \cdot \min \left(\theta, \kappa^{-1} \mu \delta_{b}^{-1} \cdot\left[\ln \delta_{b}-\ln \delta_{s}\right]+\delta_{b}^{-1} q\right)\right)\right]^{-1} } \\
& \cdot \mathbb{E}\left[\exp \left(-\kappa \mu^{-1} \delta_{b} \cdot \min \left(\theta, \kappa^{-1} \mu \delta_{b}^{-1} \cdot\left[\ln \delta_{b}-\ln \delta_{s}\right]+\delta_{b}^{-1} q\right)\right) \cdot 1_{\left\{\theta \geq \kappa^{-1} \mu \delta_{b}^{-1} \cdot\left[\ln \delta_{b}-\ln \delta_{s}\right]+\delta_{b}^{-1} q\right\}}\right] \\
\leq & 1
\end{aligned}
$$


and

$$
\begin{aligned}
& \lim _{q \rightarrow \infty} h^{\prime}(q) \\
= & {\left[\mathbb{E} \exp \left(-\kappa \mu^{-1} \delta_{b} \cdot \theta\right)\right]^{-1} \cdot \mathbb{E}\left[\exp \left(-\kappa \mu^{-1} \delta_{b} \cdot \theta\right) \cdot \lim _{q \rightarrow \infty} 1_{\left\{\theta \geq \kappa^{-1} \mu \delta_{b}^{-1} \cdot\left[\ln \delta_{b}-\ln \delta_{s}\right]+\delta_{b}^{-1} q\right\}}\right] } \\
= & 0 .
\end{aligned}
$$

Hence, $h(q)$ has a unique fixed point $q^{*}>0$. It immediately follows that the optimal security is $\min \left(\theta, D^{*}\left(q^{*}\right)\right)$.

Lemma 9 For any given $q>0$, let $s_{q}$ be an optimal security that induces information acquisition and $s(\theta)=s_{q}(\theta)+\alpha \cdot \varepsilon(\theta)$ be a perturbation of $s_{q}$, where $\varepsilon$ can be any arbitrary measurable function over $\mathbb{R}_{+}$. Then the seller's marginal expected payoff from the perturbation is given by

$$
\left.\frac{d W(s, q)}{d \alpha}\right|_{\alpha=0}=\mathbb{E}[r(\theta) \cdot \varepsilon(\theta)]
$$

where

$$
r(\theta)=-\delta_{s} m_{s_{q}, q}(\theta)+\mu^{-1} \delta_{b}\left[g^{\prime \prime}\left(m_{s_{q}, q}(\theta)\right)\right]^{-1}\left(q-\delta_{s} \cdot s_{q}(\theta)+w_{q}\right)
$$

is the seller's marginal benefit from increasing $s_{q}$ in state $\theta$, and $w_{q}$ is a constant determined in equilibrium.

Proof. I first characterize how $m_{s, q}$ responds to the perturbation of $s_{q}$. Taking derivative with respect to $\alpha$ at $\alpha=0$ for both sides of (12) and rearranging the terms lead to

$$
\left.\frac{d m_{s, q}(\theta)}{d \alpha}\right|_{\alpha=0}=\left[g^{\prime \prime}\left(m_{s_{q}, q}(\theta)\right)\right]^{-1} \cdot\left[\mu^{-1} \delta_{b} \cdot \varepsilon(\theta)+\left.g^{\prime \prime}\left(\bar{p}_{s_{q}, q}\right) \cdot \frac{d \bar{p}_{s, q}}{d \alpha}\right|_{\alpha=0}\right] .
$$

This equation clearly shows two impacts of $\varepsilon$, the perturbation of $s$, on $m_{s, q}(\theta)$. The first term in the square brackets of the right-hand side characterizes the impact of $\varepsilon(\theta)$, the local perturbation in state $\theta$. Intuitively, this impact is of the same sign as $\varepsilon(\theta)$, since increasing or decreasing the security payoff in state $\theta$ (i.e., $\varepsilon(\theta)>0$ or $\varepsilon(\theta)<0$ ) directly raises or reduces the probability of acceptance in this state. In addition, the second term in the square brackets captures the impact of the perturbation over all states as a whole. This is because $\bar{p}_{s, q}$, the unconditional probability of acceptance, aggregates $m_{s, q}(\cdot)$ over all states and thus depends on $\varepsilon$ globally. In particular, by taking expectation of both sides of (37) and making use of the fact $\left.\frac{d \bar{p}_{s, q}}{d \alpha}\right|_{\alpha=0}=\left.\mathbb{E} \frac{d m_{s, q}(\theta)}{d \alpha}\right|_{\alpha=0}$, I obtain the response of $\bar{p}_{s, q}$ to $\varepsilon$

$$
\left.\frac{d \bar{p}_{s, q}}{d \alpha}\right|_{\alpha=0}=\frac{\mu^{-1} \delta_{b} \mathbb{E}\left(\left[g^{\prime \prime}\left(m_{s_{q}, q}(\theta)\right)\right]^{-1} \varepsilon(\theta)\right)}{1-\mathbb{E}\left[g^{\prime \prime}\left(m_{s_{q}, q}(\theta)\right)\right]^{-1} \cdot g^{\prime \prime}\left(\bar{p}_{s_{q}, q}\right)} .
$$


Plugging the expression of $\left.\frac{d \bar{p}_{s, q}}{d \alpha}\right|_{\alpha=0}$ into (37) leads to

$$
\begin{aligned}
& \left.\frac{d m_{s, q}(\theta)}{d \alpha}\right|_{\alpha=0} \\
= & {\left[g^{\prime \prime}\left(m_{s_{q}, q}(\theta)\right)\right]^{-1}\left[\mu^{-1} \delta_{b} \cdot \varepsilon(\theta)+\frac{\mu^{-1} \delta_{b} \mathbb{E}\left(\left[g^{\prime \prime}\left(m_{s_{q}, q}(\theta)\right)\right]^{-1} \varepsilon(\theta)\right)}{\left[g^{\prime \prime}\left(\bar{p}_{s_{q}, q}\right)\right]^{-1}-\mathbb{E}\left[g^{\prime \prime}\left(m_{s_{q}, q}(\theta)\right)\right]^{-1}}\right], }
\end{aligned}
$$

which fully characterizes the response of $m_{s, q}$ to any perturbation $\varepsilon$ perturbation of $s_{q}$.

Now I can calculate the response of the seller's expected payoff to the perturbation of $s_{q}$. Taking the derivative of $W(s, q)$ with respect to $\alpha$ at $\alpha=0$ leads to

$$
\left.\frac{d W(s, q)}{d \alpha}\right|_{\alpha=0}=-\delta_{s} \cdot \mathbb{E}\left[m_{s_{q}, q}(\theta) \varepsilon(\theta)\right]+\mathbb{E}\left(\left.\frac{d m_{s, q}(\theta)}{d \alpha}\right|_{\alpha=0}\left[q-\delta_{s} \cdot s_{q}(\theta)\right]\right) .
$$

Here, the first term captures the direct effect of perturbing the security payoff $s_{q}$ disregarding the variation of $m_{s, q}$; the second term is its indirect effect through the variation of $m_{s, q}$. Substitute (38) into (39), and I get (35) and (36). In particular, $w_{q}$, the constant in equilibrium is given by

$$
w_{q}=\frac{\mathbb{E}\left(\left[q-\delta_{s} \cdot s_{q}(\theta)\right]\left[g^{\prime \prime}\left(m_{s_{q}, q}(\theta)\right)\right]^{-1}\right)}{\left[g^{\prime \prime}\left(\bar{p}_{s_{q}, q}\right)\right]^{-1}-\mathbb{E}\left[g^{\prime \prime}\left(m_{s_{q}, q}(\theta)\right)\right]^{-1}} .
$$

\section{Proof of Proposition 5.}

\section{Proof. Let}

$$
\begin{gathered}
A_{0}=\left\{\theta \in \Theta: \theta>0, s_{q}(\theta)=0\right\}, \\
A_{1}=\left\{\theta \in \Theta: \theta \neq 0, s_{q}(\theta) \in(0, \theta)\right\}
\end{gathered}
$$

and

$$
A_{2}=\left\{\theta \in \Theta: \theta>0, s_{q}(\theta)=\theta\right\} .
$$

Clearly, $\left\{A_{0}, A_{1}, A_{2}\right\}$ is a partition of $\Theta \backslash\{0\}$. Since $s_{q}$ is the optimal security, I obtain

$$
\left.\frac{d W(s, q)}{d \alpha}\right|_{\alpha=0} \leq 0
$$


for any feasible perturbation $\varepsilon(\theta)$. Hence, condition (35) implies

$$
r(\theta)\left\{\begin{array}{ll}
\leq 0 & \text { if } \theta \in A_{0} \\
=0 & \text { if } \theta \in A_{1} \\
\geq 0 & \text { if } \theta \in A_{2}
\end{array} .\right.
$$

Since $g$ is strictly convex, $r(\theta) \cdot g^{\prime \prime}\left(m_{s_{q}, q}(\theta)\right)$ is of the same sign as $r(\theta)$. Thus (41) can be rewritten as

$$
\begin{aligned}
& r(\theta) \cdot g^{\prime \prime}\left(m_{s_{q}, q}(\theta)\right) \\
= & -\delta_{s} m_{s_{q}, q}(\theta) g^{\prime \prime}\left(m_{s_{q}, q}(\theta)\right)+\mu^{-1} \delta_{b}\left(q-\delta_{s} \cdot s_{q}(\theta)+w_{q}\right) \\
& \begin{cases}\leq 0 & \text { if } \theta \in A_{0} \\
=0 & \text { if } \theta \in A_{1} \\
\geq 0 & \text { if } \theta \in A_{2}\end{cases}
\end{aligned}
$$

Recall condition (12), given the optimal offer $\left(s_{q}, q\right)$, the buyer's best response $m_{s_{q}, q}(\theta)$ is characterized by

$$
\delta_{b} \cdot s_{q}(\theta)-q=\mu \cdot\left[g^{\prime}\left(m_{s_{q}, q}(\theta)\right)-g^{\prime}\left(\bar{p}_{s_{q}, q}\right)\right],
$$

where

$$
\bar{p}_{s_{q}, q}=\mathbb{E} m_{s_{q}, q}(\theta)
$$

is the buyer's unconditional probability of accepting offer $\left(s_{q}, q\right)$. Conditions (42)and (43) as a system of functional equations jointly determine the optimal security $s_{q} \cdot{ }^{20}$

I solve the system of equations to get the seller's optimal security $s_{q}$ and the buyer's associated optimal decision rule of information acquisition $m_{s_{q}, q}$. To facilitate the analysis and economize on notations, consider two equivalent equations with respect to variables $m$ and $s$, in which $m$ stands for $m_{s_{q}, q}(\theta)$ and $s$ stands for $s_{q}(\theta)$ :

$$
-\delta_{s} \cdot m \cdot g^{\prime \prime}(m)+\mu^{-1} \delta_{b}\left(q-\delta_{s} \cdot s+w_{q}\right)=0
$$

and

$$
\delta_{b} \cdot s-q=\mu \cdot\left[g^{\prime}(m)-g^{\prime}\left(\bar{p}_{s_{q}, q}\right)\right] .
$$

Let $m=f_{1}(s)$ and $m=f_{2}(s)$ be the two continuous functions implicitly defined by (44) and (45), respectively.

First note that $f_{1}^{\prime}(s)<0$ and $f_{2}^{\prime}(s)>0$. This is because $\left[m \cdot g^{\prime \prime}(m)\right]^{\prime}>0$ and $g^{\prime \prime}(m)>$ 0 . Therefore, the curves $m=f_{1}(s)$ and $m=f_{2}(s)$ intersect at most once.

\footnotetext{
${ }^{20}$ One may object that Equation (42) is just the first order condition of the seller's optimization problem. It only characterizes the critical points. In principle, I should characterize the largest critical point, but the argument holds for any critical point, so the results are not open to this critique.
} 
Second, it must be true that $f_{1}(0)>f_{2}(0)$. Otherwise, $f_{1}(s)<f_{2}(s)$ for all $s>0$. Hence $\forall \theta>0$,

$$
\begin{aligned}
& r(\theta) \cdot g^{\prime \prime}\left(m_{s_{q}, q}(\theta)\right) \\
= & -\delta_{s} \cdot f_{2}\left(s_{q}(\theta)\right) g^{\prime \prime}\left(f_{2}\left(s_{q}(\theta)\right)\right)+\mu^{-1} \delta_{b}\left(q-\delta_{s} \cdot s_{q}(\theta)+w_{q}\right) \\
< & -\delta_{s} \cdot f_{1}\left(s_{q}(\theta)\right) g^{\prime \prime}\left(f_{1}\left(s_{q}(\theta)\right)\right)+\mu^{-1} \delta_{b}\left(q-\delta_{s} \cdot s_{q}(\theta)+w_{q}\right) \\
= & 0
\end{aligned}
$$

where the inequality holds since $\left[m \cdot g^{\prime \prime}(m)\right]^{\prime}>0$. Then $(42)$ implies $s_{q}(\theta)=0$ for all $\theta \in \Theta$. Therefore, there is no trade, which contradicts Proposition 3.

Third, it must be true that $\operatorname{Pr}\left(A_{0}\right)=0$. This is because, for any $\theta \in A_{0}$,

$$
\begin{aligned}
& r(\theta) \cdot g^{\prime \prime}\left(m_{s_{q}, q}(\theta)\right) \\
= & -\delta_{s} \cdot f_{2}(0) g^{\prime \prime}\left(f_{2}(0)\right)+\mu^{-1} \delta_{b}\left(q-\delta_{s} \cdot 0+w_{q}\right) \\
> & -\delta_{s} \cdot f_{1}(0) g^{\prime \prime}\left(f_{1}(0)\right)+\mu^{-1} \delta_{b}\left(q-\delta_{s} \cdot 0+w_{q}\right) \\
= & 0,
\end{aligned}
$$

where the inequality holds since $f_{1}(0)>f_{2}(0)$ and $\left[m \cdot g^{\prime \prime}(m)\right]^{\prime}>0$. This result contradicts (42), which states $r(\theta) \cdot g^{\prime \prime}\left(m_{s_{q}, q}(\theta)\right) \leq 0$ for $\theta \in A_{0}$.

Now I am ready to show that the optimal security must be debt. If $f_{1}(s)$ and $f_{2}(s)$ never intersect, $r(\theta)>0$ for all $\theta \in \Theta$. Then the optimal security

$$
s_{q}(\theta)=\theta
$$

is a special debt with a very large face value $\sup \Theta$, which may be infinity.

Let $(\bar{s}, \bar{m})$ be the unique intersection of $f_{1}(s)$ and $f_{2}(s)$. For any $\theta$ such that $\theta<\bar{s}$, the following inequality holds,

$$
m_{s_{q}, q}(\theta)=f_{2}\left(s_{q}(\theta)\right)<f_{2}(\bar{s})=f_{1}(\bar{s})<f_{1}\left(s_{q}(\theta)\right)
$$

Then

$$
\begin{aligned}
& r(\theta) \cdot g^{\prime \prime}\left(m_{s_{q}, q}(\theta)\right) \\
> & -\delta_{s} \cdot f_{1}\left(s_{q}(\theta)\right) \cdot g^{\prime \prime}\left(f_{1}\left(s_{q}(\theta)\right)\right)+\mu^{-1} \delta_{b}\left(q-\delta_{s} \cdot s_{q}(\theta)+w_{q}\right) \\
= & 0
\end{aligned}
$$

where the inequality holds since $\left[m \cdot g^{\prime \prime}(m)\right]^{\prime}>0$. Condition (42) then implies

$$
s_{q}(\theta)=\theta
$$


for all $\theta<\bar{s}$.

For any $\theta>\bar{s}$, if $s_{q}(\theta)=\theta$, then (42) implies

$$
\begin{aligned}
0 & \leq r(\theta) \cdot g^{\prime \prime}\left(m_{s_{q}, q}(\theta)\right) \\
& =-\delta_{s} \cdot f_{2}\left(s_{q}(\theta)\right) \cdot g^{\prime \prime}\left(f_{2}\left(s_{q}(\theta)\right)\right)+\mu^{-1} \delta_{b}\left(q-\delta_{s} \cdot s_{q}(\theta)+w_{q}\right) \\
& <-\delta_{s} \cdot f_{2}(\bar{s}) \cdot g^{\prime \prime}\left(f_{2}(\bar{s})\right)+\mu^{-1} \delta_{b}\left(q-\delta_{s} \cdot s_{q}(\theta)+w_{q}\right) \\
& <-\delta_{s} \cdot f_{1}\left(s_{q}(\theta)\right) \cdot g^{\prime \prime}\left(f_{1}\left(s_{q}(\theta)\right)\right)+\mu^{-1} \delta_{b}\left(q-\delta_{s} \cdot s_{q}(\theta)+w_{q}\right) \\
& =0
\end{aligned}
$$

which is a contradiction. Hence $\operatorname{Pr}\left(A_{0}\right)=0$ implies $s_{q}(\theta)=\bar{s}$ for all $\theta>\bar{s}$.

Therefore, the optimal security is a debt with face value $\bar{s}$, i.e.,

$$
s_{q}(\theta)=\min (\theta, \bar{s}) .
$$

This concludes the proof.

This proof shows that for any given price $q>0$, the optimal security $s_{q}$ is a debt security. It immediately follows that in the optimal contract $\left(s^{*}, q^{*}\right), s^{*}=s_{q^{*}}$ is a debt security, denoted by $s^{*}(\theta)=\min \left(\theta, D^{*}\right)$. Similar variational argument with respect to $q$ and $D$ leads to the following implicit characterization:

$$
\begin{gathered}
\delta_{s} m_{s^{*}, q^{*}}\left(D^{*}\right) g^{\prime \prime}\left(m_{s^{*}, q^{*}}\left(D^{*}\right)\right)-\delta_{b} \bar{p}_{s^{*}, q^{*}} g^{\prime \prime}\left(\bar{p}_{s^{*}, q^{*}}\right)=\mu^{-1} \delta_{b}\left(q^{*}-\delta_{s} D^{*}\right) \\
\delta_{b} \cdot \min \left(\theta, D^{*}\right)-q^{*}=\mu \cdot\left[g^{\prime}\left(m_{s^{*}, q^{*}}(\theta)\right)-g^{\prime}\left(\bar{p}_{s^{*}, q^{*}}\right)\right] \\
\bar{p}_{s^{*}, q^{*}}=\mathbb{E} m_{s^{*}, q^{*}}(\theta)
\end{gathered}
$$

and

$$
\bar{p}_{s^{*}, q^{*}}\left[1-g^{\prime \prime}\left(\bar{p}_{s^{*}, q^{*}}\right) \mathbb{E}\left(\frac{1}{g^{\prime \prime}\left(m_{s^{*}, q^{*}}(\theta)\right)}\right)\right]=\mu^{-1} \mathbb{E}\left(\frac{q^{*}-\delta_{s} \cdot \min \left(\theta, D^{*}\right)}{g^{\prime \prime}\left(m_{s^{*}, q^{*}}(\theta)\right)}\right) .
$$

\section{Proof of Proposition 6.}

Proof. The proof is similar to that for the baseline model. I outline the main steps here.

i) Since $\mathbb{E}(\theta)=k$, the seller can choose a security with security payoff $s(\theta)$ slightly below $\theta$ such that Condition ii) c) of Proposition 2 holds. Then the buyer acquires information and accepts the offer with positive probability. Since the seller has nothing to lose but can earn $\theta-s(\theta)$ when the buyer accepts, her expected payoff is positive. Hence her optimal security too necessarily generates a positive expected payoff, which can be achieved only through a successful transaction.

ii) If $s^{*}(\theta)=\theta$ almost surely, the seller's expected payoff is zero, which contradicts the 
optimality of $s^{*}$ according to the argument of i).

iii) By Proposition 2, $m_{s^{*}}$, the buyer's response to the optimal security $s^{*}$, is characterized by

$$
s^{*}(\theta)-k=\mu \cdot\left[g^{\prime}\left(m_{s^{*}}(\theta)\right)-g^{\prime}\left(\bar{p}_{s^{*}}\right)\right],
$$

where

$$
\bar{p}_{s^{*}}=\mathbb{E}\left[m_{s^{*}}(\theta)\right]
$$

is the buyer's unconditional probability of acceptance.

Consider an arbitrary perturbation to the optimal security,

$$
s(\theta)=s^{*}(\theta)+\alpha \cdot \varepsilon(\theta) .
$$

The seller's expected payoff from proposing $s$ is

$$
W(s)=\mathbb{E}\left[m_{s}(\theta) \cdot(\theta-s(\theta))\right] .
$$

So the seller's marginal expected payoff from adding perturbation $\varepsilon$ to the optimal security is

$$
\left.\frac{d W(s)}{d \alpha}\right|_{\alpha=0}=\mathbb{E}[r(\theta) \cdot \varepsilon(\theta)]
$$

where

$$
r(\theta)=-m_{s^{*}}(\theta)+\mu^{-1}\left[g^{\prime \prime}\left(m_{s^{*}}(\theta)\right)\right]^{-1}\left(\theta-s^{*}(\theta)+w^{*}\right)
$$

is the seller's marginal benefit from increasing $s^{*}$ in state $\theta$, and

$$
w^{*}=\mathbb{E}\left[\left(\theta-s^{*}(\theta)\right) \frac{g^{\prime \prime}\left(\bar{p}_{s^{*}}\right)}{g^{\prime \prime}\left(m_{s^{*}}(\theta)\right)}\right]\left(1-\mathbb{E}\left[\frac{g^{\prime \prime}\left(\bar{p}_{s^{*}}\right)}{g^{\prime \prime}\left(m_{s^{*}}(\theta)\right)}\right]\right)^{-1}
$$

is a constant determined in equilibrium.

Let

$$
\begin{gathered}
A_{0}=\left\{\theta \in \Theta: \theta>0, s^{*}(\theta)=0\right\}, \\
A_{1}=\left\{\theta \in \Theta: \theta>0, s^{*}(\theta) \in(0, \theta)\right\}
\end{gathered}
$$

and

$$
A_{2}=\left\{\theta \in \Theta: \theta>0, s^{*}(\theta)=\theta\right\} .
$$

The optimality of $s^{*}$ requires

$$
r(\theta) \begin{cases}\leq 0 & \text { if } \theta \in A_{0} \\ =0 & \text { if } \theta \in A_{1} \\ \geq 0 & \text { if } \theta \in A_{2}\end{cases}
$$


Multiply both sides by $g^{\prime \prime}\left(m_{s^{*}}(\theta)\right)>0$ and I obtain

$$
\begin{aligned}
r(\theta) \cdot g^{\prime \prime}\left(m_{s^{*}}(\theta)\right)= & -m_{s^{*}}(\theta) g^{\prime \prime}\left(m_{s^{*}}(\theta)\right)+\mu^{-1}\left(\theta-s^{*}(\theta)+w^{*}\right) \\
& \begin{cases}\leq 0 & \text { if } \theta \in A_{0} \\
=0 & \text { if } \theta \in A_{1} \\
\geq 0 & \text { if } \theta \in A_{2}\end{cases}
\end{aligned}
$$

Note that $r(\theta) \cdot g^{\prime \prime}\left(m_{s^{*}}(\theta)\right)-\mu^{-1} \theta$ strictly decreases in the value of $s^{*}(\theta)$. This is because the second term of the right-hand side of (49) strictly decreases in the value of $s^{*}(\theta)$, and so does the first term, which decreases in the value of $m_{s^{*}}(\theta)$ that is increasing in the value of $s^{*}(\theta)$, according to (46). I show three facts that will be used later in the proof.

Fact a) $\theta \in A_{0}$ implies $(0, \theta] \subset A_{0}$.

Let $\theta^{\prime} \in(0, \theta)$, then

$$
\begin{aligned}
r\left(\theta^{\prime}\right) \cdot g^{\prime \prime}\left(m_{s^{*}}\left(\theta^{\prime}\right)\right) & <-m_{s^{*}}\left(\theta^{\prime}\right) g^{\prime \prime}\left(m_{s^{*}}\left(\theta^{\prime}\right)\right)+\mu^{-1}\left(\theta-s^{*}\left(\theta^{\prime}\right)+w^{*}\right) \\
& \leq-m_{s^{*}}(\theta) g^{\prime \prime}\left(m_{s^{*}}(\theta)\right)+\mu^{-1}\left(\theta-s^{*}(\theta)+w^{*}\right) \\
& \leq 0
\end{aligned}
$$

where the first inequality follows $\theta^{\prime}<\theta$; the second one follows that $r(\theta) \cdot g^{\prime \prime}\left(m_{s^{*}}(\theta)\right)-\mu^{-1} \theta$ strictly decreases in the value of $s^{*}(\theta)$ and $s^{*}\left(\theta^{\prime}\right) \geq 0=s^{*}(\theta)$; and the third one follows $\theta \in A_{0}$ and $r(\theta) \leq 0$. Hence, $s^{*}\left(\theta^{\prime}\right)=0$ and $\theta^{\prime} \in A_{0}$.

Fact b) $\theta \in A_{2}$ implies $(0, \theta] \subset A_{2}$.

Let $\theta^{\prime} \in(0, \theta)$, then

$$
\begin{aligned}
r\left(\theta^{\prime}\right) \cdot g^{\prime \prime}\left(m_{s^{*}}\left(\theta^{\prime}\right)\right) & >-m_{s^{*}}(\theta) g^{\prime \prime}\left(m_{s^{*}}(\theta)\right)+\mu^{-1}\left(\theta^{\prime}-s^{*}\left(\theta^{\prime}\right)+w^{*}\right) \\
& \geq-\left[1-m_{s^{*}}(\theta)\right]^{-1}+\mu^{-1}\left(\theta-s^{*}(\theta)+w^{*}\right) \\
& \geq 0,
\end{aligned}
$$

where the first inequality follows that $-\left[1-m_{s^{*}}(\theta)\right]^{-1}$ strictly decreases in the value of $s^{*}(\theta)$ and $s^{*}\left(\theta^{\prime}\right) \leq \theta^{\prime}<\theta=s^{*}(\theta)$; the second one follows that $\theta^{\prime}-s^{*}\left(\theta^{\prime}\right) \geq 0=\theta-s^{*}(\theta)$; and the third one follows $\theta \in A_{2}$ and $r(\theta) \geq 0$. Hence, $s^{*}\left(\theta^{\prime}\right)=\theta$ and $\theta^{\prime} \in A_{2}$.

Facts a) and b) imply that $A_{0}$ and $A_{2}$ cannot be non-empty simultaneously. In addition, if $A_{2} \neq \phi$, there exists some $D^{*}$ such that $A_{2}=\left(0, D^{*}\right]$ and $A_{1}=\left(D^{*}, \infty\right)$. Note that $D^{*}=$ $\sup A_{2}<\infty$. Otherwise, $s^{*}(\theta)=\theta$ almost surely, which contradicts ii). If $A_{0} \neq \phi$, there exists some $D^{*}$ such that $A_{0}=\left(0, D^{*}\right]$ and $A_{1}=\left(D^{*}, \infty\right)$. Note that $D^{*}=\sup A_{0}<\infty$. Otherwise, $s^{*}(\theta)=0$ almost surely and the buyer simply rejects the offer, which contradicts i).

Fact c) $\frac{d s^{*}(\theta)}{d \theta} \in(0,1)$ for $\theta \in A_{1}$. 
Since $\theta \in A_{1}, r(\theta)=0$, i.e.,

$$
m_{s^{*}}(\theta)=1-\frac{\mu}{\theta-s^{*}(\theta)+w^{*}} .
$$

Plug (50) into (46) and I obtain

$$
s^{*}(\theta)-k=\mu \cdot\left[g^{\prime}\left(1-\frac{\mu}{\left(\theta-s^{*}(\theta)+w^{*}\right)}\right)-g^{\prime}\left(\bar{p}_{s^{*}}\right)\right] .
$$

Taking derivative of both sides of the the above equation and rearranging the terms lead to

$$
\begin{aligned}
\frac{d s^{*}(\theta)}{d \theta} & =\frac{\mu}{\theta-s^{*}(\theta)+w^{*}} \\
& =1-m_{s^{*}}(\theta) \in(0,1),
\end{aligned}
$$

where the second equality follows (50).

Now I show $A_{0}=\phi$. If this not true, let $A_{0}=\left(0, D^{*}\right]$ and $A_{1}=\left(D^{*}, \infty\right)$ with $D^{*}>$ 0 . Note that, $s^{*}(\theta)$ is strictly increasing when $\theta \geq D^{*}$. Also, the fact that information acquisition occurs implies that there must exist a $\theta^{\prime \prime}>D^{*}$ such that $s^{*}\left(\theta^{\prime \prime}\right)>k$; otherwise the optimal security would be rejected without information acquisition. Therefore, there exists a $\theta^{\prime}>D^{*}$ such that $s^{*}\left(\theta^{\prime}\right)=k$. Recall equation (46), I obtain

$$
m_{s^{*}}\left(\theta^{\prime}\right)=\bar{p}_{s^{*}}
$$

In addition, since $\theta^{\prime}>D^{*}$, it must be true that

$$
\begin{aligned}
0 & =r\left(\theta^{\prime}\right) \\
& =-m_{s^{*}}\left(\theta^{\prime}\right)+\mu^{-1} m_{s^{*}}\left(\theta^{\prime}\right)\left[1-m_{s^{*}}\left(\theta^{\prime}\right)\right]\left(\theta^{\prime}-s^{*}\left(\theta^{\prime}\right)+w^{*}\right) \\
& =-\bar{p}_{s^{*}}+\mu^{-1} \bar{p}_{s^{*}}\left[1-\bar{p}_{s^{*}}\right]\left(\theta^{\prime}-k+w^{*}\right) .
\end{aligned}
$$

By (48), I obtain

$$
\mathbb{E}[r(\theta)]=-\bar{p}_{s^{*}}+\mu^{-1} \bar{p}_{s^{*}}\left[1-\bar{p}_{s^{*}}\right] \cdot w^{*},
$$

which, together with (51), implies

$$
\mathbb{E}[r(\theta)]=-\mu^{-1} \bar{p}_{s^{*}}\left[1-\bar{p}_{s^{*}}\right]\left(\theta^{\prime}-k\right) .
$$

Since $\frac{d s^{*}(\theta)}{d \theta} \in(0,1)$, it must be true that $\theta^{\prime}>D^{*}+k$. Hence

$$
\mathbb{E}[r(\theta)]<-\mu^{-1} \bar{p}_{s^{*}}\left[1-\bar{p}_{s^{*}}\right] \cdot D^{*} .
$$

I can express $\mathbb{E}[r(\theta)]$ in another way. Since for all $\theta \leq D^{*}, s^{*}(\theta)=0=s^{*}\left(D^{*}\right)$ and 


$$
\begin{aligned}
m_{s^{*}}(\theta)= & m_{s^{*}}\left(D^{*}\right), \text { I obtain } \\
r(\theta) & =-m_{s^{*}}(\theta)+\mu^{-1} m_{s^{*}}(\theta)\left[1-m_{s^{*}}(\theta)\right]\left(\theta-s^{*}(\theta)+w^{*}\right) \\
& =-m_{s^{*}}\left(D^{*}\right)+\mu^{-1} m_{s^{*}}\left(D^{*}\right)\left[1-m_{s^{*}}\left(D^{*}\right)\right]\left(\theta-D^{*}+D^{*}-s^{*}\left(D^{*}\right)+w^{*}\right) \\
& =\mu^{-1} m_{s^{*}}\left(D^{*}\right)\left[1-m_{s^{*}}\left(D^{*}\right)\right]\left(\theta-D^{*}\right)
\end{aligned}
$$

where the last equality follows $r\left(D^{*}\right)=0$. Also note that $r(\theta)=0$ over $\left[D^{*}, \infty\right)$, hence

$$
\mathbb{E}[r(\theta)]=-\mu^{-1} m_{s^{*}}\left(D^{*}\right)\left[1-m_{s^{*}}\left(D^{*}\right)\right] \int_{0}^{D^{*}}\left(D^{*}-\theta\right) d P(\theta) .
$$

Combining (52) and (53) leads to

$$
\begin{aligned}
& m_{s^{*}}\left(D^{*}\right)\left[1-m_{s^{*}}\left(D^{*}\right)\right] \int_{0}^{D^{*}}\left(D^{*}-\theta\right) d P(\theta) \\
> & \bar{p}_{s^{*}}\left[1-\bar{p}_{s^{*}}\right] \cdot D^{*} \\
\geq & \int_{0}^{\infty} m_{s^{*}}(\theta)\left[1-m_{s^{*}}(\theta)\right] d P(\theta) \cdot D^{*} \\
> & m_{s^{*}}\left(D^{*}\right)\left[1-m_{s^{*}}\left(D^{*}\right)\right] \int_{0}^{D^{*}} D^{*} d P(\theta),
\end{aligned}
$$

where the second inequality follows the concavity of $x \cdot(1-x)$ and Jensen's inequality, the third inequality follows the fact that $m_{s^{*}}(\theta)=m_{s^{*}}\left(D^{*}\right)$ for all $\theta \in\left[0, D^{*}\right]$. Inequality (54) immediately implies

$$
-m_{s^{*}}\left(D^{*}\right)\left[1-m_{s^{*}}\left(D^{*}\right)\right] \int_{0}^{D^{*}} \theta d P(\theta)>0
$$

which is a contradiction. Therefore, I showed $A_{0}=\phi$.

Finally, Facts a), b) and c) imply that there exists a $D^{*} \geq 0$, such that $\frac{d s^{*}(\theta)}{d \theta}=1$ on $\left[0, D^{*}\right]$ and $\frac{d s^{*}(\theta)}{d \theta} \in(0,1)$ on $\left[D^{*}, \infty\right)$. This concludes the proof.

\section{Proof of Proposition 7.}

Proof. Let $s(\theta)=s_{q}(\theta)+\alpha \cdot \varepsilon(\theta)$ be a perturbation of $s_{q}$, where $\varepsilon$ can be any arbitrary measurable function over $\mathbb{R}_{+}$. I first characterize how $m_{s, q}$ responds to the perturbation of $s_{q}$. Since I have shown that $\eta(\theta) \leq 0$, the buyer's optimal strategy to $(s, q)$ is characterized by

$$
\mu^{-1}\left[\delta_{b} s(\theta)-q\right]=g^{\prime}\left(m_{s, q}(\theta)\right)-g^{\prime}\left(\bar{p}_{s, q}\right)+\min \left(\mu^{-1}\left[\delta_{b} s(\theta)-q\right]-g^{\prime}(0)+g^{\prime}\left(\bar{p}_{s, q}\right), 0\right)
$$

Taking derivative with respect to $\alpha$ at $\alpha=0$ for both sides of (55) and rearranging the 
terms lead to

$$
\left.\frac{d m_{s, q}(\theta)}{d \alpha}\right|_{\alpha=0}=\left[g^{\prime \prime}\left(m_{s_{q}, q}(\theta)\right)\right]^{-1} \cdot 1_{B} \cdot\left[\mu^{-1} \delta_{b} \cdot \varepsilon(\theta)+\left.g^{\prime \prime}\left(\bar{p}_{s_{q}, q}\right) \cdot \frac{d \bar{p}_{s, q}}{d \alpha}\right|_{\alpha=0}\right],
$$

where

$$
B=\left\{\theta \in \Theta: \mu^{-1}\left[\delta_{b} s_{q}(\theta)-q\right]>g^{\prime}(0)-g^{\prime}\left(\bar{p}_{s_{q}, q}\right)\right\}
$$

is the event that transaction happens, i.e., $m_{s_{q}, q}(\theta)>0$, and $1_{B}$ is the indicator function for set $B$. By taking expectation of both sides of (56) and making use of the fact $\left.\frac{d \bar{p}_{s, q}}{d \alpha}\right|_{\alpha=0}=$ $\left.\mathbb{E} \frac{d m_{s, q}(\theta)}{d \alpha}\right|_{\alpha=0}$, I obtain the response of $\bar{p}_{s, q}$ to $\varepsilon$

$$
\left.\frac{d \bar{p}_{s, q}}{d \alpha}\right|_{\alpha=0}=\frac{\mu^{-1} \delta_{b} \mathbb{E}\left(\left[1_{B} \cdot g^{\prime \prime}\left(m_{s_{q}, q}(\theta)\right)\right]^{-1} \varepsilon(\theta)\right)}{1-\mathbb{E}\left[\frac{1_{B}}{g^{\prime \prime}\left(m_{s_{q}, q}(\theta)\right)}\right] \cdot g^{\prime \prime}\left(\bar{p}_{s_{q}, q}\right)}
$$

Plugging the expression of $\left.\frac{d \bar{p}_{s, q}}{d \alpha}\right|_{\alpha=0}$ into (56) leads to

$$
\begin{aligned}
& \left.\frac{d m_{s, q}(\theta)}{d \alpha}\right|_{\alpha=0} \\
= & 1_{B}\left[g^{\prime \prime}\left(m_{s_{q}, q}(\theta)\right)\right]^{-1}\left[\mu^{-1} \delta_{b} \cdot \varepsilon(\theta)+\frac{\mu^{-1} \delta_{b} \mathbb{E}\left(1_{B}\left[g^{\prime \prime}\left(m_{s_{q}, q}(\theta)\right)\right]^{-1} \varepsilon(\theta)\right)}{\left[g^{\prime \prime}\left(\bar{p}_{s_{q}, q}\right)\right]^{-1}-\mathbb{E}\left[\frac{1_{B}}{g^{\prime \prime}\left(m_{s_{q}, q}(\theta)\right)}\right]}\right]
\end{aligned}
$$

which fully characterizes the response of $m_{s, q}$ to any perturbation $\varepsilon$ perturbation of $s_{q}$.

Now I can calculate the response of the seller's expected payoff to the perturbation of $s_{q}$. Taking the derivative of $W(s, q)$ with respect to $\alpha$ at $\alpha=0$ leads to

$$
\left.\frac{d W(s, q)}{d \alpha}\right|_{\alpha=0}=-\delta_{s} \cdot \mathbb{E}\left[m_{s_{q}, q}(\theta) \varepsilon(\theta)\right]+\mathbb{E}\left(\left.\frac{d m_{s, q}(\theta)}{d \alpha}\right|_{\alpha=0}\left[q-\delta_{s} \cdot s_{q}(\theta)\right]\right) .
$$

Here, the first term captures the direct effect of perturbing the security payoff $s_{q}$ disregarding the variation of $m_{s, q}$; the second term is its indirect effect through the variation of $m_{s, q}$. Substitute (57) into (58), and I obtain

$$
\left.\frac{d W(s, q)}{d \alpha}\right|_{\alpha=0}=\mathbb{E}[r(\theta) \cdot \varepsilon(\theta)]
$$

where

$$
r(\theta)=-\delta_{s} m_{s_{q}, q}(\theta)+\mu^{-1} \delta_{b} 1_{B}\left[g^{\prime \prime}\left(m_{s_{q}, q}(\theta)\right)\right]^{-1}\left(q-\delta_{s} \cdot s_{q}(\theta)+w_{q}\right)
$$


In particular, $w_{q}$, the constant in equilibrium is given by

$$
w_{q}=\frac{\mathbb{E}\left(\left[q-\delta_{s} \cdot s_{q}(\theta)\right] 1_{B}\left[g^{\prime \prime}\left(m_{s_{q}, q}(\theta)\right)\right]^{-1}\right)}{\left[g^{\prime \prime}\left(\bar{p}_{s_{q}, q}\right)\right]^{-1}-\mathbb{E}\left[\frac{1_{B}}{g^{\prime \prime}\left(m_{s_{q}, q}(\theta)\right)}\right]} .
$$

Let

$$
\begin{gathered}
A_{0}=\left\{\theta \in B: \theta>0, s_{q}(\theta)=0\right\}, \\
A_{1}=\left\{\theta \in B: \theta \neq 0, s_{q}(\theta) \in(0, \theta)\right\}
\end{gathered}
$$

and

$$
A_{2}=\left\{\theta \in B: \theta>0, s_{q}(\theta)=\theta\right\}
$$

Clearly, $\left\{A_{0}, A_{1}, A_{2}\right\}$ is a partition of $B \backslash\{0\}$. Since $s_{q}$ is the optimal security, I obtain

$$
\left.\frac{d W(s, q)}{d \alpha}\right|_{\alpha=0} \leq 0
$$

for any feasible perturbation $\varepsilon(\theta)$. Hence, condition (59) implies

$$
r(\theta)\left\{\begin{array}{ll}
\leq 0 & \text { if } \theta \in A_{0} \\
=0 & \text { if } \theta \in A_{1} \\
\geq 0 & \text { if } \theta \in A_{2}
\end{array} .\right.
$$

Since $g$ is strictly convex, $r(\theta) \cdot g^{\prime \prime}\left(m_{s_{q}, q}(\theta)\right)$ is of the same sign as $r(\theta)$. Thus (62) can be rewritten as

$$
\begin{aligned}
& r(\theta) \cdot g^{\prime \prime}\left(m_{s_{q}, q}(\theta)\right) \\
= & -\delta_{s} m_{s_{q}, q}(\theta) g^{\prime \prime}\left(m_{s_{q}, q}(\theta)\right)+\mu^{-1} \delta_{b}\left(q-\delta_{s} \cdot s_{q}(\theta)+w_{q}\right) \\
& \begin{cases}\leq 0 & \text { if } \theta \in A_{0} \\
=0 & \text { if } \theta \in A_{1} \\
\geq 0 & \text { if } \theta \in A_{2}\end{cases}
\end{aligned}
$$

Recall Condition (55), given the optimal offer $\left(s_{q}, q\right)$, for $\theta \in B$ the buyer's best response $m_{s_{q}, q}(\theta)$ is characterized by

$$
\delta_{b} \cdot s_{q}(\theta)-q=\mu \cdot\left[g^{\prime}\left(m_{s_{q}, q}(\theta)\right)-g^{\prime}\left(\bar{p}_{s_{q}, q}\right)\right],
$$

where

$$
\bar{p}_{s_{q}, q}=\mathbb{E} m_{s_{q}, q}(\theta)
$$

is the buyer's unconditional probability of accepting offer $\left(s_{q}, q\right)$. Conditions (63)and (64) 
as a system of functional equations jointly determine the optimal security $s_{q}$.

I solve the system of equations to obtain the seller's optimal security $s_{q}$ and the buyer's associated optimal decision rule of information acquisition $m_{s_{q}, q}$. To facilitate the analysis and economize on notations, consider two equivalent equations with respect to variables $m$ and $s$, in which $m$ stands for $m_{s_{q}, q}(\theta)$ and $s$ stands for $s_{q}(\theta)$ :

$$
-\delta_{s} \cdot m \cdot g^{\prime \prime}(m)+\mu^{-1} \delta_{b}\left(q-\delta_{s} \cdot s+w_{q}\right)=0
$$

and

$$
\delta_{b} \cdot s-q=\mu \cdot\left[g^{\prime}(m)-g^{\prime}\left(\bar{p}_{s_{q}, q}\right)\right] .
$$

Let $m=f_{1}(s)$ and $m=f_{2}(s)$ be the two continuous functions implicitly defined by (65) and (66), respectively.

First note that $f_{1}^{\prime}(s)<0$ and $f_{2}^{\prime}(s)>0$. This is because $\left[m \cdot g^{\prime \prime}(m)\right]^{\prime}>0$ and $g^{\prime \prime}(m)>$ 0 . Therefore, the curves $m=f_{1}(s)$ and $m=f_{2}(s)$ intersect at most once.

Second, it must be true that $f_{1}(0)>f_{2}(0)$. Otherwise, $f_{1}(s)<f_{2}(s)$ for all $s>0$. Hence $\forall \theta>0$,

$$
\begin{aligned}
& r(\theta) \cdot g^{\prime \prime}\left(m_{s_{q}, q}(\theta)\right) \\
= & -\delta_{s} \cdot f_{2}\left(s_{q}(\theta)\right) g^{\prime \prime}\left(f_{2}\left(s_{q}(\theta)\right)\right)+\mu^{-1} \delta_{b}\left(q-\delta_{s} \cdot s_{q}(\theta)+w_{q}\right) \\
< & -\delta_{s} \cdot f_{1}\left(s_{q}(\theta)\right) g^{\prime \prime}\left(f_{1}\left(s_{q}(\theta)\right)\right)+\mu^{-1} \delta_{b}\left(q-\delta_{s} \cdot s_{q}(\theta)+w_{q}\right) \\
= & 0
\end{aligned}
$$

where the inequality holds since $\left[m \cdot g^{\prime \prime}(m)\right]^{\prime}>0$. Then $(63)$ implies $s_{q}(\theta)=0$ for all $\theta \in B$. Therefore, there is no trade, contradicting the assumption of this proposition.

Third, it must be true that $\operatorname{Pr}\left(A_{0}\right)=0$. This is because, for any $\theta \in A_{0}$,

$$
\begin{aligned}
& r(\theta) \cdot g^{\prime \prime}\left(m_{s_{q}, q}(\theta)\right) \\
= & -\delta_{s} \cdot f_{2}(0) g^{\prime \prime}\left(f_{2}(0)\right)+\mu^{-1} \delta_{b}\left(q-\delta_{s} \cdot 0+w_{q}\right) \\
> & -\delta_{s} \cdot f_{1}(0) g^{\prime \prime}\left(f_{1}(0)\right)+\mu^{-1} \delta_{b}\left(q-\delta_{s} \cdot 0+w_{q}\right) \\
= & 0,
\end{aligned}
$$

where the inequality holds since $f_{1}(0)>f_{2}(0)$ and $\left[m \cdot g^{\prime \prime}(m)\right]^{\prime}>0$. This result contradicts (63), which states $r(\theta) \cdot g^{\prime \prime}\left(m_{s_{q}, q}(\theta)\right) \leq 0$ for $\theta \in A_{0}$.

Now I am ready to show that the optimal security must be debt on event $B$. If $f_{1}(s)$ and $f_{2}(s)$ never intersect, $r(\theta)>0$ for all $\theta \in B$. Then the optimal security

$$
s_{q}(\theta)=\theta
$$


is a special debt with a very large face value $\sup B$, which may be infinity.

Let $(\bar{s}, \bar{m})$ be the unique intersection of $f_{1}(s)$ and $f_{2}(s)$. For any $\theta$ such that $\theta<\bar{s}$, the following inequality holds,

$$
m_{s_{q}, q}(\theta)=f_{2}\left(s_{q}(\theta)\right)<f_{2}(\bar{s})=f_{1}(\bar{s})<f_{1}\left(s_{q}(\theta)\right)
$$

Then

$$
\begin{aligned}
& r(\theta) \cdot g^{\prime \prime}\left(m_{s_{q}, q}(\theta)\right) \\
> & -\delta_{s} \cdot f_{1}\left(s_{q}(\theta)\right) \cdot g^{\prime \prime}\left(f_{1}\left(s_{q}(\theta)\right)\right)+\mu^{-1} \delta_{b}\left(q-\delta_{s} \cdot s_{q}(\theta)+w_{q}\right) \\
= & 0,
\end{aligned}
$$

where the inequality holds since $\left[m \cdot g^{\prime \prime}(m)\right]^{\prime}>0$. Condition (63) then implies

$$
s_{q}(\theta)=\theta
$$

for all $\theta<\bar{s}$.

For any $\theta>\bar{s}$, if $s_{q}(\theta)=\theta$, then (63) implies

$$
\begin{aligned}
0 & \leq r(\theta) \cdot g^{\prime \prime}\left(m_{s_{q}, q}(\theta)\right) \\
& =-\delta_{s} \cdot f_{2}\left(s_{q}(\theta)\right) \cdot g^{\prime \prime}\left(f_{2}\left(s_{q}(\theta)\right)\right)+\mu^{-1} \delta_{b}\left(q-\delta_{s} \cdot s_{q}(\theta)+w_{q}\right) \\
& <-\delta_{s} \cdot f_{2}(\bar{s}) \cdot g^{\prime \prime}\left(f_{2}(\bar{s})\right)+\mu^{-1} \delta_{b}\left(q-\delta_{s} \cdot s_{q}(\theta)+w_{q}\right) \\
& <-\delta_{s} \cdot f_{1}\left(s_{q}(\theta)\right) \cdot g^{\prime \prime}\left(f_{1}\left(s_{q}(\theta)\right)\right)+\mu^{-1} \delta_{b}\left(q-\delta_{s} \cdot s_{q}(\theta)+w_{q}\right) \\
& =0
\end{aligned}
$$

which is a contradiction. Hence $\operatorname{Pr}\left(A_{0}\right)=0$ implies $s_{q}(\theta)=\bar{s}$ for all $\theta>\bar{s}$.

Therefore, the optimal security is a debt with face value $\bar{s}$, i.e.,

$$
s_{q}(\theta)=\min (\theta, \bar{s})
$$

This concludes the proof.

\section{Proof of Proposition 8.}

Proof. Note that the proof of Proposition 2 applies to any abstract probability space $\Theta$. Especially, in this proof I apply it to $\Theta=\mathbb{R}_{+}^{N}$.

i) Again, I prove by constructing a debt security that generates positive expected payoff to the seller. Let $\beta \in\left(\delta_{s} \delta_{b}^{-1}, 1\right)$ and

$$
f(q)=\mathbb{E} \min \left(\sum_{n=1}^{N} \theta_{n}, \beta \delta_{s}^{-1} q\right) .
$$


Since $P$ is a continuous distribution and $\beta^{-1} \delta_{s} \delta_{b}^{-1}<1$, there exists $q_{0}>0$ s.t.

$$
\operatorname{Pr}\left(\sum_{n=1}^{N} \theta_{n} \geq \beta \delta_{s}^{-1} q\right)>\beta^{-1} \delta_{s} \delta_{b}^{-1}
$$

for all $q \in\left[0, q_{0}\right]$. Hence for any $q \in\left(0, q_{0}\right)$,

$$
\begin{aligned}
f^{\prime}(q) & =\operatorname{Pr}\left(\sum_{n=1}^{N} \theta_{n} \geq \beta \delta_{s}^{-1} q\right) \cdot \beta \delta_{s}^{-1} \\
& >\beta^{-1} \delta_{s} \delta_{b}^{-1} \cdot \beta \delta_{s}^{-1}=\delta_{b}^{-1} .
\end{aligned}
$$

Note that

$$
f(0)=0
$$

which implies that

$$
f(q)>\delta_{b}^{-1} q
$$

for all $q \in\left(0, q_{0}\right)$.

Consider a debt

$$
s(\vec{\theta})=\min \left(\sum_{n=1}^{N} \theta_{n}, D\right)
$$

with face value $D=\beta \delta_{s}^{-1} q$ and price $q \in\left(0, q_{0}\right)$. The buyer's payoff gain from accepting this offer over rejecting it is

$$
\Delta u(\vec{\theta})=\delta_{b} \cdot s(\vec{\theta})-q .
$$

By Jensen's inequality, I obtain

$$
\begin{aligned}
& \mathbb{E} \exp \left(\mu^{-1} \Delta u(\vec{\theta})\right) \\
\geq & \exp \left(\mu^{-1} \mathbb{E} \Delta u(\vec{\theta})\right) \\
> & 1,
\end{aligned}
$$

which implies $\bar{p}_{s, q}>0$ according to Proposition 2. Then, the seller's expected payoff from this offer is

$$
\begin{aligned}
W(s, q) & =\mathbb{E}\left(m_{s, q}(\vec{\theta}) \cdot\left[q-\delta_{s} \cdot s(\vec{\theta})\right]\right) \\
& \geq \mathbb{E}\left(m_{s, q}(\vec{\theta}) \cdot\left[q-\delta_{s} \cdot \beta \delta_{s}^{-1} q\right]\right) \\
& =(1-\beta) q \cdot \bar{p}_{s, q}>0 .
\end{aligned}
$$

By proposing the optimal offer $\left(s^{*}, q^{*}\right)$, the seller's expected payoff should be no less than $W(s, q)>0$. This directly implies $\bar{p}_{s^{*}, q^{*}}>0$, since $\bar{p}_{s^{*}, q^{*}}=0$ always generates zero 
expected payoff to the seller.

ii) I first discuss the case without information acquisition. Similar to (10), the price of security $s(\vec{\theta})$ is given by

$$
q=-\mu \ln \mathbb{E} \exp \left(-\mu^{-1} \delta_{b} \cdot s(\vec{\theta})\right)
$$

Let $s(\vec{\theta})=s^{*}(\vec{\theta})+\alpha \cdot \varepsilon(\vec{\theta})$ be an arbitrary perturbation of the optimal security $s^{*}$. Let

$$
J(\alpha)=\mu \ln \mathbb{E} \exp \left(-\mu^{-1} \delta_{b} \cdot s(\vec{\theta})\right)+\delta_{s} \cdot \mathbb{E} s(\vec{\theta}) .
$$

Taking first order variation leads to

$$
\begin{aligned}
& \left.\frac{d J}{d \alpha}\right|_{\alpha=0} \\
= & \mathbb{E}\left[\left(\delta_{s}-\delta_{b}\left[\mathbb{E} \exp \left(-\mu^{-1} \delta_{b} \cdot s^{*}(\vec{\theta})\right)\right]^{-1} \exp \left(-\mu^{-1} \delta_{b} \cdot s^{*}(\vec{\theta})\right)\right) \cdot \varepsilon(\vec{\theta})\right] \\
\triangleq & \mathbb{E}[r(\vec{\theta}) \cdot \varepsilon(\vec{\theta})] .
\end{aligned}
$$

Let

$$
\begin{gathered}
A_{0}=\left\{\vec{\theta} \in \Theta: \vec{\theta} \neq \overrightarrow{0}, s^{*}(\vec{\theta})=0\right\} \\
A_{1}=\left\{\vec{\theta} \in \Theta: \vec{\theta} \neq \overrightarrow{0}, s^{*}(\vec{\theta}) \in\left(0, \sum_{n=1}^{N} \theta_{n}\right)\right\}
\end{gathered}
$$

and

$$
A_{2}=\left\{\vec{\theta} \in \Theta: \vec{\theta} \neq \overrightarrow{0}, s^{*}(\vec{\theta})=\sum_{n=1}^{N} \theta_{n}\right\} .
$$

Since $s^{*}$ is the optimal security,

$$
\left.\frac{d J}{d \alpha}\right|_{\alpha=0} \geq 0
$$

holds for any feasible perturbation $\varepsilon(\cdot)$. Hence, I obtain

$$
r(\vec{\theta}) \begin{cases}\geq 0 & \text { if } \vec{\theta} \in A_{0} \\ =0 & \text { if } \vec{\theta} \in A_{1} \\ \leq 0 & \text { if } \vec{\theta} \in A_{2}\end{cases}
$$

For any $\vec{\theta}^{\prime} \in A_{0},(69)$ implies $r\left(\vec{\theta}^{\prime}\right) \geq 0$, i.e.,

$$
\delta_{s} \geq \delta_{b}\left[\mathbb{E} \exp \left(-\mu^{-1} \delta_{b} \cdot s^{*}(\vec{\theta})\right)\right]^{-1}
$$


Together with (67), this inequality implies

$$
\ln \delta_{s} \geq \ln \delta_{b}+\mu^{-1} q^{*}
$$

Hence,

$$
\mu^{-1} q^{*} \leq \ln \delta_{s}-\ln \delta_{b}<0,
$$

which is a contradiction. Therefore,

$$
\operatorname{Pr}\left(A_{0}\right)=0 .
$$

For any $\vec{\theta}^{\prime} \in A_{1},(69)$ implies $r\left(\vec{\theta}^{\prime}\right)=0$, i.e.,

$$
\delta_{s}=\delta_{b}\left[\mathbb{E} \exp \left(-\mu^{-1} \delta_{b} \cdot s^{*}(\vec{\theta})\right)\right]^{-1} \exp \left(-\mu^{-1} \delta_{b} \cdot s^{*}\left(\vec{\theta}^{\prime}\right)\right),
$$

i.e.,

$$
\ln \delta_{s}=\ln \delta_{b}+\mu^{-1} q^{*}-\mu^{-1} \delta_{b} \cdot s^{*}\left(\vec{\theta}^{\prime}\right)
$$

Therefore,

$$
s^{*}\left(\vec{\theta}^{\prime}\right)=\mu \delta_{b}^{-1} \cdot\left[\ln \delta_{b}-\ln \delta_{s}\right]+\delta_{b}^{-1} q^{*}
$$

is a constant for all $\vec{\theta}^{\prime} \in A_{1}$.

For any $\vec{\theta}^{\prime} \in A_{2},(69)$ implies $r\left(\vec{\theta}^{\prime}\right) \leq 0$, i.e.,

$$
\delta_{s} \leq \delta_{b}\left[\mathbb{E} \exp \left(-\mu^{-1} \delta_{b} \cdot s^{*}(\vec{\theta})\right)\right]^{-1} \exp \left(-\mu^{-1} \delta_{b} \cdot \sum_{n=1}^{N} \theta_{n}^{\prime}\right)
$$

i.e.,

$$
\ln \delta_{s} \leq \ln \delta_{b}+\mu^{-1} q^{*}-\mu^{-1} \delta_{b} \cdot \sum_{n=1}^{N} \theta_{n}^{\prime}
$$

Therefore,

$$
\sum_{n=1}^{N} \theta_{n}^{\prime} \leq \mu \delta_{b}^{-1} \cdot\left[\ln \delta_{b}-\ln \delta_{s}\right]+\delta_{b}^{-1} q^{*}
$$

Let

$$
D^{*}=\mu \delta_{b}^{-1} \cdot\left[\ln \delta_{b}-\ln \delta_{s}\right]+\delta_{b}^{-1} q^{*} .
$$

Then, (70), (71) and (72) imply that

$$
s^{*}(\vec{\theta})=\min \left(\sum_{n=1}^{N} \theta_{n}, D^{*}\right),
$$


i.e., the optimal security must be a debt.

Now I discuss the case with information acquisition. The same derivations in the proof of Lemma 9 lead to

$$
\left.\frac{d W\left(s, q^{*}\right)}{d \alpha}\right|_{\alpha=0}=\mathbb{E}[r(\vec{\theta}) \cdot \varepsilon(\vec{\theta})]
$$

where

$$
r(\vec{\theta})=-\delta_{s} m_{s^{*}, q^{*}}(\vec{\theta})+\mu^{-1} \delta_{b}\left[g^{\prime \prime}\left(m_{s^{*}, q^{*}}(\vec{\theta})\right)\right]^{-1}\left(q-\delta_{s} \cdot s^{*}(\vec{\theta})+w^{*}\right)
$$

and

$$
w^{*}=\frac{\mathbb{E}\left(\left[q^{*}-\delta_{s} \cdot s^{*}(\vec{\theta})\right]\left[g^{\prime \prime}\left(m_{s^{*}, q^{*}}(\vec{\theta})\right)\right]^{-1}\right)}{\left[g^{\prime \prime}\left(\bar{p}_{s^{*}, q^{*}}\right)\right]^{-1}-\mathbb{E}\left[g^{\prime \prime}\left(m_{s^{*}, q^{*}}(\vec{\theta})\right)\right]^{-1}} .
$$

Again, let

$$
\begin{gathered}
A_{0}=\left\{\vec{\theta} \in \Theta: \vec{\theta} \neq \overrightarrow{0}, s^{*}(\vec{\theta})=0\right\} \\
A_{1}=\left\{\vec{\theta} \in \Theta: \vec{\theta} \neq \overrightarrow{0}, s^{*}(\vec{\theta}) \in\left(0, \sum_{n=1}^{N} \theta_{n}\right)\right\}
\end{gathered}
$$

and

$$
A_{2}=\left\{\vec{\theta} \in \Theta: \vec{\theta} \neq \overrightarrow{0}, s^{*}(\vec{\theta})=\sum_{n=1}^{N} \theta_{n}\right\} .
$$

Since $s^{*}$ is the optimal security, it must be true that

$$
\left.\frac{d W\left(s, q^{*}\right)}{d \alpha}\right|_{\alpha=0} \leq 0
$$

for any feasible perturbation $\varepsilon(\vec{\theta})$. Hence, condition (73) implies

$$
r(\vec{\theta}) \begin{cases}\leq 0 & \text { if } \vec{\theta} \in A_{0} \\ =0 & \text { if } \vec{\theta} \in A_{1} \\ \geq 0 & \text { if } \vec{\theta} \in A_{2}\end{cases}
$$

Since $g$ is strictly convex, $r(\vec{\theta}) \cdot g^{\prime \prime}\left(m_{s^{*}, q^{*}}(\vec{\theta})\right)$ is of the same sign as $r(\vec{\theta})$. Thus (75) 
can be rewritten as

$$
\begin{aligned}
& r(\vec{\theta}) \cdot g^{\prime \prime}\left(m_{s^{*}, q^{*}}(\vec{\theta})\right) \\
= & -\delta_{s} m_{s^{*}, q^{*}}(\vec{\theta}) g^{\prime \prime}\left(m_{s^{*}, q^{*}}(\vec{\theta})\right)+\mu^{-1} \delta_{b}\left(q^{*}-\delta_{s} \cdot s^{*}(\vec{\theta})+w^{*}\right) \\
& \begin{cases}\leq 0 & \text { if } \vec{\theta} \in A_{0} \\
=0 & \text { if } \vec{\theta} \in A_{1} \\
\geq 0 & \text { if } \vec{\theta} \in A_{2}\end{cases}
\end{aligned}
$$

According to Proposition 2, given the optimal offer $\left(s^{*}, q^{*}\right)$, the buyer's best response $m_{s^{*}, q^{*}}(\vec{\theta})$ is characterized by

$$
\delta_{b} \cdot s^{*}(\vec{\theta})-q^{*}=\mu \cdot\left[g^{\prime}\left(m_{s^{*}, q^{*}}(\vec{\theta})\right)-g^{\prime}\left(\bar{p}_{s^{*}, q^{*}}\right)\right],
$$

where

$$
\bar{p}_{s^{*}, q^{*}}=\mathbb{E} m_{s^{*}, q^{*}}(\vec{\theta}) .
$$

Conditions (76)and (77) as a system of functional equations jointly determine the optimal security $s^{*}$. To facilitate the analysis and economize on notations, consider two equivalent equations with respect to variables $m$ and $s$, in which $m$ stands for $m_{s^{*}, q^{*}}(\vec{\theta})$ and $s$ stands for $s^{*}(\vec{\theta})$ :

$$
-\delta_{s} \cdot m \cdot g^{\prime \prime}(m)+\mu^{-1} \delta_{b}\left(q^{*}-\delta_{s} \cdot s+w^{*}\right)=0
$$

and

$$
\delta_{b} \cdot s-q^{*}=\mu \cdot\left[g^{\prime}(m)-g^{\prime}\left(\bar{p}_{s^{*}, q^{*}}\right)\right] .
$$

Let $m=f_{1}(s)$ and $m=f_{2}(s)$ be the two continuous functions implicitly defined by (78) and (79), respectively. As before, it is clear that $f_{1}^{\prime}(s)<0$ and $f_{2}^{\prime}(s)>0$. So the curves $m=f_{1}(s)$ and $m=f_{2}(s)$ intersect at most once.

First note that $f_{1}(0)>f_{2}(0)$. Otherwise, $f_{1}(s)<f_{2}(s)$ for all $s>0$. Hence $\forall \vec{\theta} \neq \overrightarrow{0}$,

$$
\begin{aligned}
& r(\vec{\theta}) \cdot g^{\prime \prime}\left(m_{s^{*}, q^{*}}(\vec{\theta})\right) \\
= & -\delta_{s} \cdot f_{2}\left(s^{*}(\vec{\theta})\right) g^{\prime \prime}\left(f_{2}\left(s^{*}(\vec{\theta})\right)\right)+\mu^{-1} \delta_{b}\left(q^{*}-\delta_{s} \cdot s^{*}(\vec{\theta})+w^{*}\right) \\
< & -\delta_{s} \cdot f_{1}\left(s^{*}(\vec{\theta})\right) g^{\prime \prime}\left(f_{1}\left(s^{*}(\vec{\theta})\right)\right)+\mu^{-1} \delta_{b}\left(q^{*}-\delta_{s} \cdot s^{*}(\vec{\theta})+w^{*}\right) \\
= & 0
\end{aligned}
$$

where the inequality holds since $\left[m \cdot g^{\prime \prime}(m)\right]^{\prime}>0$. Then $(76)$ implies $s^{*}(\vec{\theta})=0$ for all $\vec{\theta} \in \Theta$. Therefore, there is no trade, which contradicts i) of this Proposition. 
Second note that $\operatorname{Pr}\left(A_{0}\right)=0$. This is because, for any $\vec{\theta} \in A_{0}$,

$$
\begin{aligned}
& r(\vec{\theta}) \cdot g^{\prime \prime}\left(m_{s^{*}, q^{*}}(\vec{\theta})\right) \\
= & -\delta_{s} \cdot f_{2}(0) g^{\prime \prime}\left(f_{2}(0)\right)+\mu^{-1} \delta_{b}\left(q^{*}-\delta_{s} \cdot 0+w^{*}\right) \\
> & -\delta_{s} \cdot f_{1}(0) g^{\prime \prime}\left(f_{1}(0)\right)+\mu^{-1} \delta_{b}\left(q^{*}-\delta_{s} \cdot 0+w^{*}\right) \\
= & 0,
\end{aligned}
$$

where the inequality holds since $f_{1}(0)>f_{2}(0)$ and $\left[m \cdot g^{\prime \prime}(m)\right]^{\prime}>0$. This result contradicts (76), which states $r(\vec{\theta}) \cdot g^{\prime \prime}\left(m_{s^{*}, q^{*}}(\vec{\theta})\right) \leq 0$ for $\vec{\theta} \in A_{0}$.

Now I am ready to show that the optimal security must be debt. If $f_{1}(s)$ and $f_{2}(s)$ never intersect, $r(\vec{\theta})>0$ for all $\vec{\theta} \in \Theta$. Then the optimal security

$$
s^{*}(\vec{\theta})=\sum_{n=1}^{N} \theta_{n}
$$

is a special debt with a very large face value $\sup \left\{\sum_{n=1}^{N} \theta_{n}: \vec{\theta} \in \Theta\right\}$, which may be infinity.

Let $(\bar{s}, \bar{m})$ be the unique intersection of $f_{1}(s)$ and $f_{2}(s)$. For any $\vec{\theta}$ such that $\sum_{n=1}^{N} \theta_{n}<$ $\bar{s}$, the following inequality holds,

$$
m_{s^{*}, q^{*}}(\vec{\theta})=f_{2}\left(s^{*}(\vec{\theta})\right)<f_{2}(\bar{s})=f_{1}(\bar{s})<f_{1}\left(s^{*}(\vec{\theta})\right) .
$$

Then

$$
\begin{aligned}
& r(\vec{\theta}) \cdot g^{\prime \prime}\left(m_{s^{*}, q^{*}}(\vec{\theta})\right) \\
> & -\delta_{s} \cdot f_{1}\left(s^{*}(\vec{\theta})\right) \cdot g^{\prime \prime}\left(f_{1}\left(s^{*}(\vec{\theta})\right)\right)+\mu^{-1} \delta_{b}\left(q^{*}-\delta_{s} \cdot s^{*}(\vec{\theta})+w\right) \\
= & 0
\end{aligned}
$$

where the inequality holds since $\left[m \cdot g^{\prime \prime}(m)\right]^{\prime}>0$. Condition (76) then implies

$$
s^{*}(\vec{\theta})=\sum_{n=1}^{N} \theta_{n}
$$

for all $\vec{\theta}$ such that $\sum_{n=1}^{N} \theta_{n}<\bar{s}$. 
For any $\vec{\theta}$ such that $\sum_{n=1}^{N} \theta_{n}>\bar{s}$, if $s^{*}(\vec{\theta})=\sum_{n=1}^{N} \theta_{n}$, then (76) implies

$$
\begin{aligned}
0 & \leq r(\vec{\theta}) \cdot g^{\prime \prime}\left(m_{s^{*}, q^{*}}(\vec{\theta})\right) \\
& =-\delta_{s} \cdot f_{2}\left(s^{*}(\vec{\theta})\right) \cdot g^{\prime \prime}\left(f_{2}\left(s^{*}(\vec{\theta})\right)\right)+\mu^{-1} \delta_{b}\left(q^{*}-\delta_{s} \cdot s^{*}(\vec{\theta})+w\right) \\
& <-\delta_{s} \cdot f_{2}(\bar{s}) \cdot g^{\prime \prime}\left(f_{2}(\bar{s})\right)+\mu^{-1} \delta_{b}\left(q^{*}-\delta_{s} \cdot s^{*}(\vec{\theta})+w\right) \\
& <-\delta_{s} \cdot f_{1}\left(s^{*}(\vec{\theta})\right) \cdot g^{\prime \prime}\left(f_{1}\left(s^{*}(\vec{\theta})\right)\right)+\mu^{-1} \delta_{b}\left(q^{*}-\delta_{s} \cdot s^{*}(\vec{\theta})+w\right) \\
& =0
\end{aligned}
$$

which is a contradiction. Hence $\operatorname{Pr}\left(A_{0}\right)=0$ implies $s^{*}(\vec{\theta})=\bar{s}$ for all $\vec{\theta}$ such that $\sum_{n=1}^{N} \theta_{n}>\bar{s}$.

For any $\vec{\theta}$ such that $\sum_{n=1}^{N} \theta_{n}=\bar{s}, s^{*}(\vec{\theta})=\bar{s}$ is a direct implication of $\operatorname{Pr}\left(A_{0}\right)=0$.

Therefore, the optimal security is a debt with face value $\bar{s}$, i.e.,

$$
s^{*}(\vec{\theta})=\min \left(\sum_{n=1}^{N} \theta_{n}, \bar{s}\right) .
$$

